\title{
Utilization of marine by-products for the recovery of value-added products
}

\author{
Fereidoon Shahidi*, Vamadevan Varatharajan\#, \\ Han Peng and Ruchira Senadheera
}

\begin{abstract}
Department of Biochemistry, Memorial University of Newfoundland, St. John's, Newfoundland, A1B 3X9, Canada
\#Present address, Cargill $1480028^{\text {th }}$ Ave. N., Plymouth, MN 55447, USA.

${ }^{*}$ Corresponding author: Fereidoon Shahidi, Department of Biochemistry, Memorial University of Newfoundland, St. John's, Newfoundland, A1B 3X9, Canada. Tel: 709-864-8552; Fax: 709-864-4000; E-mail: fshahidi@mun.ca
\end{abstract}

DOI: $10.31665 / J F B .2019 .6184$

Received: May 29, 2019; Revised received \& accepted: June 25, 2019

Citation: Shahidi, F., Varatharajan, V., Peng, H., and Senadheera, R. (2019). Utilization of marine by-products for the recovery of valueadded products. J. Food Bioact. 6: 10-61.

\begin{abstract}
The world fisheries resources have exceeded 160 million tons in recent years. However, every year a considerable amount of total catch is discarded as by-catch or as processing leftovers, and that includes trimmings, fins, frames, heads, skin, viscera and among others. In addition, a large quantity of processing by-products is accumulated as shells of crustaceans and shellfish from marine bioprocessing plants. Recognition of the limited marine resources and the increasing environmental pollution has emphasized the need for better utilization of the by-products. Marine by-products contain valuable protein and lipid fractions, minerals, enzymes as well as many other components. The major fraction of by-products are used for feed production-in making fish meal/oil, but this has low profitability. However, there are many ways in which the fish and shellfish waste could be better utilized, including the production of novel food ingredients, nutraceuticals, pharmaceuticals, biomedical materials, fine chemicals, and other value-added products. In recent times, much research is conducted in order to explore the possible uses of different by-products. This contribution primarily covers the characteristics and utilization of the main ingredients such as protein, lipid, chitin and its derivatives, enzymes, carotenoids, and minerals originating from marine by-products.
\end{abstract}

Keywords: Fish; Shellfish; By-products; By-catch; Discards; Processing; Added-value; Nutraceutical; Omega-3 oil; Biopeptide; Chitosan.

\section{Introduction}

The living marine resources are limited, therefore, it is important to utilize them in a sustainable manner. According to FAO (2012, 2018a, b), marine fisheries production has been around 108 million tons in recent years (Figure 1) and this is expected to continue increasing in 2018 with aquaculture harvests now accounting for around 49 percent of the total. As for food fish, tighter supplies for a number of key wild-caught species including cod, herring and cephalopods will contribute to further reduction in capture fisheries to around $45 \%$ while projected growth in global harvests of shrimp, salmon, tilapia, pangasius, seabass and seabream will contribute to a slight increase $(2.9 \%)$ in consumption of farmed fish. Overall, the expected increase of world fish traded volume is around $0.7 \%$. However, the market indications of FAO (2018c) predict that international seafood traded value is going to expand significantly $(8.3 \%)$ due to favorable economic conditions worldwide, increased demand for seafood and sharp price increases despite the on-going 'trade war' between the United States of America and China and widespread monetary tightening by central banks. As a result, every year a considerable amount of total catch is discarded as processing leftovers without consideration of their potential value-added use. In addition, segments of the industry are also faced with a tremendous amount of by-catch, which is not properly retained and 


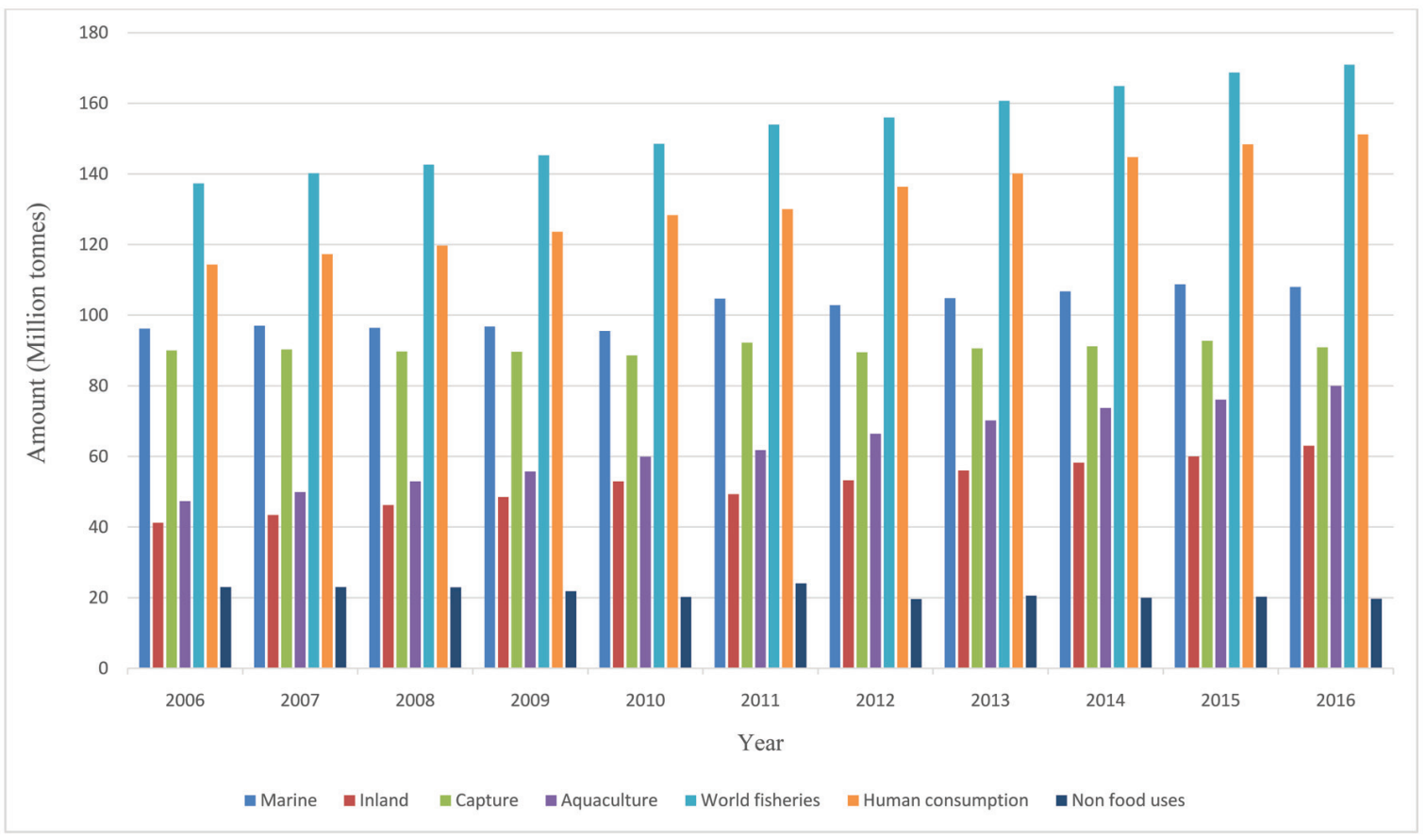

Figure 1. World fisheries and aquaculture production and utilization (FAO, 2012, 2018a).

utilized (Batista, 2007; Bimbo, 2009; Zeller et al., 2018).

Fish is an important source of animal protein as well as other nutrients for human health all over the world (Abraha et al., 2018). Meanwhile, fish is also one of the most versatile food commodities and can be utilized in a number of ways and product forms; they are generally distributed as fresh, chilled, frozen, heat-treated, fermented, dried, smoked, salted, pickled, boiled, fried, freeze-dried, minced, powdered or canned, or a combination of two or more of these forms (FAO, 2008). However, fish can be preserved and processed by many other methods. Furthermore, fish preservation and processing may vary according to the species because they have their own characteristic composition, size, shape and intrinsic chemistry (Rustad et al., 2011; Arnaud et al., 2018). Based on an early study conducted in 2005 by FAO, around 7.3 million tons of whole fish ( $\sim 8 \%$ of the global catch by volume) is discarded before landing worldwide every year in commercial fisheries (Ramírez, 2013). Overall, discards from all of these edible operations are conservatively estimated at $50 \%$ of whole fish (Taylor and Alsalvar, 2002), but can range from 10 to $90 \%$, depending on the species of fish and the intended use (Bimbo, 2009). For example, for cod in Norway, it could be $58 \%$, but mollusks, crustacean, and shellfish, in general, have a much higher proportion, for example, $88 \%$ for scallops (Ramírez, 2013). Mostly, the discards from shellfish range from 50 to $80 \%$, particularly the shrimp shell discards have increased tremendously over the past two decades (Ambigaipalan and Shahidi 2017; Taylor and Alasalvar, 2002).

In primary processing of fish and shellfish, all the edible or inedible leftover could be considered as by-products which have different qualities and potentials (Gildberg, 2002; Jaczynski, 2008; Kestin, 2017; Le Gouic et al., 2019). To obtain a final product with higher commercial value, several operations are involved in fish processing such as shelling, heading, gutting, filleting, removing tails and peeling (Ramírez, 2013). These operations generate byproducts, including heads, tails, viscera, roe, frame, shells, skin, fins, scales, blood, and among others. Moreover, by-catch, the portion of marine life caught that was not targeted, can also be processed into useful products (Kestin, 2017; Shahidi 2007; Venugopal and Shahidi, 1995). By-catch may include low-value species but also vast tonnage of undersized fish of valuable commercial species. According to UNEP (2004), almost $25 \%$ of all the fish harvested from the sea never makes it to the market. An average of 27 million tons of unwanted fish is thrown back into the ocean each year, and a large portion of it does not survive. Sometimes by-catch fish are kept for the market, but most often they are thrown back dead, because they may be the wrong species, the wrong size, of inferior quality, or surplus to the fishing operations quotas (UNEP 2004). However, according to the Landing Obligation of the European Commission Common Fisheries Policy, from 2019 onwards all fishing vessels are forced to keep all species that are caught without discarding, even though they are underutilized in commercial level (Vázquez et al., 2019). However, fish that are saleable vary considerably from country to country. Currently, by-catch is used mainly in the fishmeal industry with oil being a by-product (Shahidi 2007), and there has been a notable increase in global consumption of fish meal and fish oil during the past two decades (Fortune et al., 2018).

Fish by-products are highly perishable because of several chemical and biological changes that take place immediately after capture (Falch et al., 2007; Le Gouic et al., 2019; Vázquez et al., 2019). Moreover, during storage and transportation, the nutritional and sensory quality of these fish and their by-products may deteriorate due to the spoilage mediated by microbial and enzymatic reactions (Singh and Benjakul, 2018). In this connection, less attention has been paid to the preservation of by-products and hence conservation of their nutritional quality is one of the main challenges facing the industry. For instance, protease-mediated protein degradation should be controlled or minimized as a high degree of hydrolysis of raw materials may create bitter taste peptides (Singh and Benjakul, 2018; Alder-Nissen, 1984) and together with lipid peroxidation may cause variability in raw material (Alder-Nissen, 1984; Olsen et al., 2014). The acceptable levels of lipid and protein 
hydrolysis are highly product specific, depending on the end use of products (Falch, et al., 2007; Guérard et al., 2005). The cleaving of peptide bonds can have a negative impact on the physicochemi$\mathrm{cal}$ and functional properties of proteins due to their structural and conformational changes (Singh and Benjakul, 2018). Since these are important determinants of quality of the raw material, controlling autolysis and autoxidation of the by-products are of crucial importance for effective utilization of by-products (Rustad, 2003; Rustad et al., 2011). This will be a real challenge for the fishing vessels, demanding more advanced equipment and technology for processing and catching and more competence from harvesters for better handling to retain the quality of by-products (Batista 2007; Galanakis, 2013). Particularly, implementation of effective process control procedures, including Hazard Analysis Critical Control Point (HACCP) systems are important as these fish and their byproducts are more perishable than other muscle foods (Dehghani et al.,2018). Besides, the global seafood industry always prefers to select the options with the least investment and least penalties or consequences. The business of disposal of seafood by-products may put producers into a loss or breakeven venture in lots of areas, therefore government policies including penalty and subsidy setting also play an indispensable role (Ramírez, 2013).

Although most of the fish by-products produced in large shoreside fish processing operations are turned into low-value fish meal and crude oil, there are many other ways in which the fish and shellfish by-products could be better utilized (Galanakis, 2013; Karayannakidis and Zotos, 2016; Kurtovic, et al., 2009; Rustad et al., 2011; Shahidi, 2007; Singh and Benjakul, 2018; Venugopal and Shahidi, 1995). In recent years, considerable research has been carried out to utilize the ingredients/compounds originating from by-products of seafood industry in diverse sectors such as food, nutraceutical, functional food, pharmaceutical, biomedical, livestock, aquaculture feed, agriculture, biodiesel, and fine chemicals, among others (Taylor and Alasalvar, 2002; Ambigaipalan and Shahidi 2017; Haard, 1998; Hayes et al., 2008b; Hicks and Verbeek, 2016; Karayannakidis and Zotos, 2016; Kim and Mendis, 2006; Ohshima, 1998; Rustad et al., 2011; Shahidi, 1994, 2007; Shahidi and Kamil, 2001; Shahidi et al., 1999). However, it is more profitable to make products for human consumption while the highest profitability is in producing specialty commodities (extracting and purifying) such as enzymes, bioactive peptides, and bio-polymers for biotechnological or pharmaceutical applications (Ambigaipalan and Shahidi 2017; Kim et al., 2016; Rustad, 2003, 2011). Therefore, devising strategies for full utilization of the catch and processing of discards for production of novel products is a matter of importance for both the fishing industry and food processors (Chen et al., 2016; Shahidi, 2002). This review examines the main characteristics of ingredients, namely protein, lipid, chitin/chitosan and its derivatives, minerals, enzymes, pigments and flavor compounds originating from by-products of the seafood industry and then describes the possible ways to use them for production of value-added products.

\section{Protein}

The increasing demand for protein on a global scale turns the focus on underutilized protein sources (Rustad, 2003; Shahidi and Rahman 2018). Fish processing typically results in a large percentage of by-products, much of which is high in nutritionally desirable muscle protein (Djellouli et al., 2019; Le Gouic et al., 2019, Ockerman, 1992). These protein-rich by-products include cut-offs, backbones, heads, skin, milt, stomach, viscera, and blood, among oth- ers. For example, shrimp processing industry is producing higher amounts of by-products including carapaces, heads, and tails that account for up to $20-50 \%$ of total weight (Djellouli et al., 2019) and most of these discards are rich in different bioactive compounds. In general, fish meat and oil left on the by-products range widely, but typically account for $20-30 \mathrm{~g} / 100 \mathrm{~g}$ and $5-15 \mathrm{~g} / 100$ g, respectively (Torres et al., 2007). A wide variety of protein-rich by-products have been used for making fish silage (Aspevik et al., 2017; Gildberg, 2002; Martínez-Alvarez et al., 2015). However, recovered protein from by-products could be used for the development of human food products, dietary supplements and pharmaceuticals (Ambigaipalan and Shahidi 2017; Hayes et al., 2018; Karim and Bhat, 2009; Kim and Mendis, 2006; Wasswa, et al., 2007). Proteins could be recovered by different processes using mechanical separation from frames, base extraction, or hydrolysis (Shahidi 2007; Sila and Bougatef, 2016). Isoelectric solubilization/ precipitation has also been examined to recover muscle protein from fish by-products (Hultin and Kelleher, 1999; Tahergorabi and Ibrahim, 2016; Taskaya and Jaczynsk, 2009). This section reviews collagen/gelatin and proteoglycan, protein hydrolysate, surimi, protein concentrate, and fish silage.

\subsection{Collagen and gelatin}

The use of collagen and gelatin has expanded over a diverse field in foods (Atay et al., 2018, Kittiphattanabawon et al., 2005; Rahman 2019), pharmaceutical/biomedical and cosmetics (Senevirathne and Kim, 2012). The industrial use of collagen has been limited to porcine skin and bovine skin and bone (Badii and Howell, 2006; Choi and Regenstein, 2000; Larrazábal and Camacho, 2008). However, the bovine spongiform encephalopathy (mad-cow disease) and foot-and-mouth disease (FMD) crisis as well as religious and social issues in some countries and markets and ecological impact of wasting the fish skin are the driving force for the increasing commercial use of fish collagen (Badii and Howell, 2006; Blanco et al., 2017; Choi and Regenstein, 2000; Ennaas et al., 2016; Gilsenan and Ross-Murphy, 2000; Karim and Bhat, 2009; Kim and Mendis, 2006; Rustad, 2003; Supavititpatana et al., 2008; Wasswa, et al., 2007). This approach assures the sustainable exploitation of marine resources whilst addressing the waste disposal issues related to environmental pollution (Carvalho et al., 2018). Recent research indicates that marine organisms are the most convenient and the safest sources to obtain high-quality collagen. Therefore, marine collagens are considered as promising alternatives to mammalian collagen, particularly in biomedical applications as well as in food uses (Bernhardt et al., 2018). Apart from having desirable functional properties such as good absorption capacity (Rahman 2019), they have better potential in biomaterial applications due to a lower risk related to biological toxins. Hence, no known risk of disease transmission, minimal inflammatory responses, as well as no religious and ethical constraints account for their potential application in different areas. Therefore, collagen from marine origin can be used in a plethora of applications compared to other collagen sources, particularly terrestrial animal originated collagen. According to the current research trends, collagens of marine origin have overtaken mammalian collagens in tissue engineering of the biomedical field (Bernhardt et al., 2018;).

Fish skin, scales, bone, and fins have been widely studied as alternative sources of collagen (Bae et al., 2008; Ehrlich et al., 2018; Hernández-Briones et al., 2009; Kim et al., 2016; Kim and Mendis 2006; Nomura, et al., 1995). In addition, it has been found that the mantle of scallops (Shen et al., 2007), the muscle layer of ascidians (Mizuta et al., 2002a), and the adductor of pearl oys- 
ters (Mizuta et al., 2002b; Wu et al., 2019) can be used as new sources of collagen. Approximately, $36-54 \%$ of collagen could be obtained from fish skin, bone and fin (Nagai and Suzuki, 2000). It has been reported that $70 \%$ of the pollock skin and squid skin dry matter is collagen (Zhang et al., 2003). Some research works in Japan has demonstrated that fish scales of sardines have potential as an important collagen source (Belouafa et al., 2018; Kimura and Ohno, 1987; Nomura et al., 1996). Rohu, catla, megrim, dover sole, codfish, hake, carp, shark, spotted golden goatfish, tuna, niger triggerfish and tilapia are some of the fish species that have been subjected to extraction of collagen from marine vertebrates (Gómez-Guillén et al., 2002; Kittiphattanabawon et al., 2010; Matmaroh et al., 2011; Zhang et al., 2011; Carvalho et al., 2018). A recent study found that collagens extracted from Atlantic Salmon Salmo salar has the potential for use as scaffolds for bone tissue engineering applications (Bernhardt et al., 2018). However, the main constraint related to fish collagens is the establishment of a sound extraction process for their commercial exploitation (Carvalho et al., 2018).

The basic subunit of the collagen is tropocollagen, which is a three stranded polypeptide unit. Collagen polypeptide chains are structurally organized in three $\alpha$-helices forming its secondary structure (Silva et al., 2014). These polypeptide chains wrap around one another and form a characteristic triple helix-tertiary structure. Collagen fibrils are formed by self-assembling these triple helices. Collagen family is classified into various groups, mainly due to their complex structural diversity (Wang et al., 2014). Currently, 27 different types of collagen have been identified (Schrieber and Gareis, 2007). These collagens could simply be classified as type I (primarily in connective tissue such as skin, bone, and tendons), type II (exclusively in cartilage tissue), type III (strongly dependent on age: very young skin can contain up to $50 \%$, but in the course of time this is reduced to 5-10\%) and other types (present in very low amounts only and mostly organ-specific). Collagen molecules, composed of three $\alpha$-chains intertwined in the so-called collagen triple-helix, adopt a 3D structure that provides an ideal geometry for inter-chain hydrogen bonding, which stabilizes the triple helices (Nijenhuis, 1997). Collagen is generally extracted with mild acid or alkaline hydrolysis and solubilized without altering its triple helix (Abdollahi et al., 2018; Hernández-Briones et al., 2009; Regenstein and Zhou, 2007). The composition of collagen encompasses all 20 amino acids (Schrieber and Gareis, 2007) and it is the only mammalian protein that contains large amounts of hydroxyproline and hydroxylysine, and it is also high in its content of imino acids (proline and hydroxyproline) (Balian and Bowes, 1977). Generally, the amino acid sequence of collagen determines its functional properties including energy storage capacity, stiffness, elasticity and other mechanical properties (Uzel et al., 2009; Ehrlich et al., 2018).

Fish collagens have been shown to vary widely in their amino acid composition. In particular, the levels of proline, and hydroxyproline vary significantly among fish species (Arnesen and Gildberg, 2002; Balian and Bowes, 1977). The amount of amino acids, especially hydroxyproline, depends on the environmental temperature in which the fish lives, and this affects the thermal stability of the collagen (Balian and Bowes, 1977). Overall, collagens derived from fish have lower concentrations of imino acids (proline and hydroxyproline) compared to mammalian collagen, and warmwater fish collagen (such as bigeye-tuna and tilapia) have a higher imino acid content than cold-water fish (such as cod, whiting, and halibut) collagen (Eastoe and Leach, 1977). Hydroxyproline is involved in inter-chain hydrogen bonding, which stabilizes the triple helical structure of collagen. The collagen derived from fish species living in cold environments exhibits lower thermal stability than those from fish living in warm environments (Wasswa et al., 2007).

The high demand for potential usage of collagen has led to intense research on different marine species, mainly due to its biocompatibility, biodegradability and weak antigenic potential as biomaterial (Carvalho et al., 2018). In this respect, as mentioned earlier, marine invertebrate collagen has gained much attention as an alternative to mammalian collagen (Tziveleka et al., 2017; Ehrlich et al., 2018). Collagens from poriferans, coelenterates, annelids, mollusks, echinoderms, and crustaceans have been investigated in several studies (Silva et al., 2014; Ehrlich et al., 2018). They possess different morphological characters, and most of them are quite distinct from each other (Langasco et al., 2017). Their unique structural features, such as structure-function interrelations, have made them more specific compared to vertebrate collagen. The potential of using collagen originating from marine invertebrates in diverse fields, including food (Hashim et al., 2015), medicine (Porfírio and Fanaro, 2016; Pawelec et al., 2016; Conway et al., 2017) and cosmetics (Avilia-Rodriguez et al., 2018) has gained much momentum. However, their usage has been hindered, mainly due to difficulties in their purification and relative species-dependent complexity (Ehrlich et al., 2018).

Gelatin, not a naturally occurring protein, is derived from the fibrous protein collagen and it is a heterogeneous mixture of watersoluble proteins of high molecular weight (Budavari, 1996; Kwak et al., 2019). The global demand for gelatin has increased over the last decade (Tümerkan et al., 2019). For example, Mad-Ali et al. (2016) expected that the demand for gelatin for food and non-food industry reach 450.7 kilotons in 2018 (Huang et al., 2018). Similar to collagen, marine originated gelatin is regarded as an effective replacement to mammalian gelatin in order to avoid potential issues related to religious perspectives as well as safety and stability concerns (Kwak et al., 2019). Conversion of collagen into soluble gelatin can be achieved by heating the collagen in either acid, alkali (Wasswa et al., 2007; Schrieber and Gareis, 2007; Ahmad et al., 2017) or using enzymatic hydrolysis, the latter could also improve further degradation of gelatin into its hydrolysates. Thermal treatment destabilizes the triple helix configuration of collagen and converts its helical conformation into coiled conformation by cleaving hydrogen and covalent bonds (Djabourov et al., 1993; Kwak et al., 2019). Thus, hot water treatment is generally used to solubilize collagen in the skin and extract it as gelatin (Kim and Mendis, 2006). Silver carp skin (Boran and Regenstein 2009), rohu and tuna skins (Ninan et al.,2011), and skins from Catla catla, Labeo rohita, and Cirrhinus mrigala (Chandra and Shamasundar, 2015) are some of the examples that were used to produce gelatin from aquatic species. Moreover, tuna head bone originated gelatin showed excellent gelling properties (Haddar et al., 2012). Regenstein et al. (2003) reported that scale collagen has a unique niche market related to kocher fish. More recently, the extraction of collagen from swim bladders of fish has been considered (Liu et al., 2015).

The type of gelatin produced depends on the fish type and the production process (Wasswa et al., 2007). The gel strengths of commercial gelatins are expressed using bloom values (Schrieber and Gareis, 2007). The gelling strength of commercial gelatins ranges from 100 to 300 , but gelatins with bloom values of 250-260 are the most desirable (Holzer, 1996). Cold water fish (Pollock, haddock, cod, salmon) gelatins are particularly different due to their lower content of proline and hydroxyproline (Kwak et al., 2017) compared to mammalian derived gelatin, but these usually have poor gelling ability and their gelling temperature is typically below $8-10^{\circ} \mathrm{C}$ (Norland, 1990). Particularly, gel strength and melting temperature of fish gelatin are lower than those of mam- 
malian gelatin, mainly due to the differences in their amino acid composition (Kwak et al., 2017). Therefore, functional properties including gel strength, viscosity, gelling behavior, and the melting point of gelatin are determined by the source and type of collagen (Kamer et al.,2019). However, the viscous liquid state of the fish gelatin at room temperature is considered as a unique rheological property that can be utilized in fabricating biomaterials under "green" process conditions (Paszkiewicz et al., 2016). Warm water fish (tuna, tilapia) gelatin can have a bloom value of 200-250 $\mathrm{g}$ and their melting point is $25-27^{\circ} \mathrm{C}$ and these gelatins more closely resemble bovine or porcine gelatin, which melts at $32-35^{\circ} \mathrm{C}$ (Karim and Bhat, 2009).

There are basically two processes by which collagen is processed to gelatin, that is, acid and alkali processes (Wasswa et al., 2007) (Figure 2). However, acidic treatment is most suitable for the less covalently cross-linked collagens found in porcine and fish skins and sometimes bone raw materials (Karim and Bhrat, 2008). Pretreatment of the raw material, collagen hydrolysis to gelatin and purification and drying are the main processing steps in gelatin production (Kamer et al., 2019).

Several reports have been published about mechanical and physicochemical properties of fish gelatins obtained from several fish species (Table 1). Recently, Yan et al. (2009) investigated the physical-chemical properties of collagen from squid skin, which is 8-10 percent of the leftover in squid processing.

As discussed earlier, collagen from fish by-products has some important features for food processing that are distinguishable from those of vertebrate land sources. The important feature is fish collagen has lower denaturation temperature and viscosity than land vertebrate collagen because of lower imino acid content and a lower degree of proline hydroxylation (Kimura and Ohno, 1987; Leuenberger, 1991). Therefore, cold-water fish gelatin can be used in applications that do not require a high bloom value or gelling, relying instead on its other properties, such as prevention of syneresis and texturization (Wasswa et al., 2007). Cold-water fish gelatin can be used in frozen or refrigerated products that are consumed quickly following removal from the fridge or defrosting (Karim and Bhrat, 2008). In addition, as a protein, gelatin is low in calories, and melts in the mouth to give excellent sensory properties resembling fat, making it ideal for use in low-fat products (Karim and Bhrat, 2008). Fish gelatin may also be used in the microencapsulation of colorants (Karim and Bhrat, 2008) and food flavors (150-300 Bloom) (Soper and Thomas, 2000). Choi and Regenstein (2000) reported that fish gelatin with lower gel melting temperatures had a better release of aroma and offered a stronger flavor. Moreover, processing and extraction conditions, including time and temperature combinations, as well as the nature of chemicals can also affect the techno-functional properties of gelatin due to various lengths of polypeptide chains (Kamer et al., 2019). It has also been reported that fish gelatin emulsion is significantly affected by $\mathrm{pH}$ and storage time with large increments in consistency coefficient and particle size, mainly due to the production of relatively large droplet size during homogenization (Surh et al., 2006; Huang et al., 2018).

However, it is possible to enhance the gel properties of these gelatins by mixing with high-quality gelatins from warm-water fishes or mammals (Zhou et al., 2006) or by means of chemical and enzymatic modification (Gudmundsson, 2002; Kolodzjieska et al., 2004). Kołodziejska et al. (2004) investigated the enzymatically crosslinked fish gels and suggested that fish could be used as a gelling component of sterilized products. Furthermore, several studies have been conducted to investigate the modification of fish gelatin using transglutaminase process in order to improve the functional properties of gelatin (Jongjareonrak et al., 2006b;

\section{ACID PROCESS}

Fresh crushed raw material

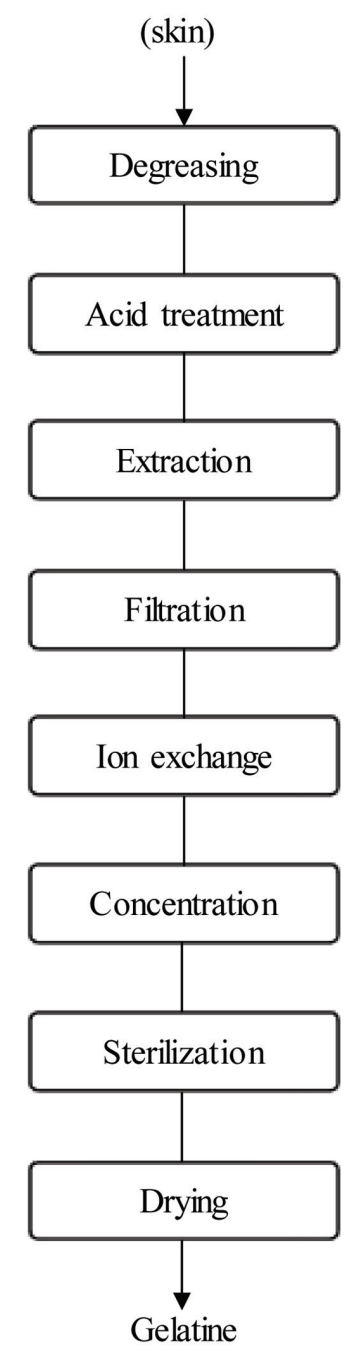

\section{ALKALI PROCESS}

Fresh crushed raw material

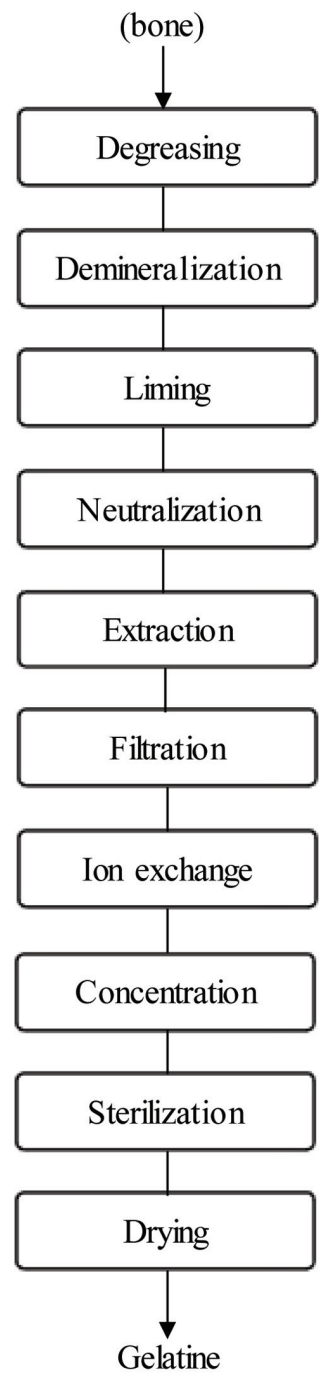

Figure 2. Flow process for most typical processing steps for fish gelatine manufacture. Source: Adapted from Wasswa et al. (2007).

Kołodziejska et al., 2004). The use of transglutaminase can enhance the elasticity and cohesiveness of the gels as well as providing non-thermoreversible gels with lower gel strength (Huang et al., 2017).

In addition, fish gelatin could be used as a food emulsifier (Surh et al., 2006), edible film (Senaratne et al., 2006; Avena-Bustillos et al., 2006), thickener, stabilizer, foaming agent (Karaman et al., 2016) and additive in surimi processing to improve the mechanical and functional properties of gels (Hernández-Briones et al., 2009). Zhou and Regenstein (2007) reported that desserts made from fish gelatins by increasing the concentration of gelatin or by using gelatin mixtures would be more similar to desserts made from high bloom pork skin gelatin. Compared to fish species, a special feature of squid collagen lies in its high degree of cross-linking, due to the high amount of hydroxylysine, together with a high content of hydroxyproline (Gómez-Guillén et al., 2002). Giménez et al. (2009) reported that gelatins from outer and inner tunics of giant 
Table 1. Examples of literature on extraction and characterization of fish gelatine

\begin{tabular}{|c|c|}
\hline Variety of fish & Reference \\
\hline $\begin{array}{l}\text { Baltic cod (Gadus morhua), salmon (Salmo } \\
\text { salar), herrings (Clupea harengus) }\end{array}$ & Kołodziejska et al. (2008) \\
\hline Flounder (Platichthys flesus) & Fernández-Díaz et al. (2003) \\
\hline Alaska pollock (Theragra chalcogramma) & Zhou and Regenstein (2005); Zhou and Regenstein (2004) \\
\hline $\begin{array}{l}\text { Alaska pollock (Theragra chalcogramma) } \\
\text { surimi processing by-products }\end{array}$ & Kim and Park (2004) \\
\hline $\begin{array}{l}\text { Megrim (Lepidorhombrus boscii) (Risso), Hake } \\
\text { (Merluccius merluccius), Dover sole (Solea vulgaris) }\end{array}$ & $\begin{array}{l}\text { Fernández-Díaz et al. (2001); Gómez-Guillén } \\
\text { et al. (2002); Sarabia et al. (2000) }\end{array}$ \\
\hline Megrim (Lepidorhombrus boscii) & Montero and Gómez-Guillén (2000) \\
\hline Grass carp (Catenopharyngodon idella) & Kasankalaa et al. (2007) \\
\hline Brown backed toadfish (Lagocephalus gloveri) & Senaratne et al. (2006) \\
\hline Horse mackerel (Trachurus trachurus) & Badii and Howell (2006) \\
\hline Cod (Gadus morhua) & $\begin{array}{l}\text { Fernández-Díaz et al. (2001); Kołodziejska et al. } \\
\text { (2004); Gudmundsson and Hafsteinsson (1997) }\end{array}$ \\
\hline Catfish (Ictalurus punctatus) & Yang et al. (2007); Liu et al. (2008) \\
\hline $\begin{array}{l}\text { Sin croaker (Johnius dussumieri), shortfin } \\
\text { scad (Decapterus macrosoma) }\end{array}$ & Cheow et al. (2007) \\
\hline $\begin{array}{l}\text { Black tilapia (Oreochromis mossambicus), } \\
\text { red tilapia (Oreochromis nilotica) }\end{array}$ & Jamilah and Harvinder (2002) \\
\hline $\begin{array}{l}\text { Bigeye snapper (Priacanthus macracanthus), } \\
\text { brownstripe red snapper (Lutjanus vitta) }\end{array}$ & Jongjareonrak et al. (2006b); Jongjareonrak et al. (2006a) \\
\hline Yellowfin tuna (Thunnus albacares) & Cho et al. (2006) \\
\hline Blue shark (Prionace glauca) & Yoshimura et al. (2000) \\
\hline Nile perch (Lates niloticus) & Muyonga et al. (2004) \\
\hline Grass carp (Ctenopharyngodon idella) & Kasankala et al. (2007) \\
\hline Skate (Raja kenojei) & Cho, Jahncke, Chin and Eun (2006) \\
\hline Atlantic salmon (Salmo salar) & Arnesen and Gildberg (2007) \\
\hline Dover sole (Solea vulgaris) & Gómez-Guillén et al. (2005); Giménez et al. (2005) \\
\hline Harp seal & Arnesen and Gildberg (2007) \\
\hline
\end{tabular}

squid exhibit good filmogenic ability. Moreover, addition of sugar can influence the functional, physicochemical, and structural properties of gelatin (Hazaveh et al., 2015; Kamer et al., 2019). It has been found that low concentrations of sugar decrease gel strength, while higher levels of sugar increase the gelling and melting temperatures (Sow and Yang 2015; Cai et al., 2017). These structural features may improve the potential application of fish gelatin in confectionery products. For example, Kamer et al. (2019) reported that addition of sorbitol and sucrose to gelatin produced from rainbow trout (Oncorhynchus mykiss) skin showed enhanced properties of fish gel solutions with confectionery solutes that have the potential to be used in gummy-like candies.

Fish gelatin has also been used in the preparation of pharmaceutical and medical products as its low gelling temperatures offer new potential application areas (Wasswa et al., 2007). One of the major applications of fish gelatin is in microencapsulation of vitamins and other pharmaceutical additives such as azoxanthine (Karim and Bhat, 2008). The use of fish gelatin soft capsules is most common in nutrition supplements. Park et al. (2007) patented a process describing the preparation of a film-forming composition for hard capsules composed of fish gelatin. Another patent (Hansen et al., 2002) described the use of fish gelatin (Bloom value higher than 100) as an ingredient in drug tablets. Moreover, the use of gelatin is generally recommended for reducing the fat levels in food formulated for obese patients (Liu et al.,2015; Huang et al., 2018).

The skin and cartilage of the blue shark are rich in types I and II collagen, respectively (Nomura, 2001). Meanwhile, type I collagen is the most commonly used and researched collagen (Lin et al., 2019). Recent studies have shown that sharkskin collagen is useful as the material for cell culture matrix, a substrate for matrix metalloprotease involved in tissue metabolism (Nomura, 2001), wound dressings, vitreous implants and carrier for drug delivery (Senaratne, Park and Kim, 2006). According to the available database, including Web of Science, there are increasing number of collagen-based biomaterials that are being approved for regenerative medicine applications (Lin et al., 2019). Several studies have reported that collagen from fresh water fish has a lower cytotoxic effect and higher cell viability than bovine collagen (Song et al., 2006; Lin et al., 2019). Furthermore, it has been reported that shark gelatin improves bone mineral density in a rat model 
(Nomura, 2004; Koyama et al., 2001). These results indicate that shark gelatin would be useful as a food supplement for treating osteoporosis. Manikandan et al. (2018) reported that gelatin from fish scales modified with caffeic acid showed better mechanical, biodegradability, and cytocompatible properties compared to their native forms and was an effective material for tissue engineering research.

However, application of fish skin collagen in food and biomaterials is still limited due to its poor solubility, negative sensory properties (fishy smell) and weak gel strength (Lin and $\mathrm{Li}$, 2006; Yan, et al., 2008). Nonetheless, more recent studies have been concentrated on developing various methods for improving cross linking and thermal stability of marine collagen (Subhan et al., 2015). It has been reported that denaturation temperature of salmon atelocollagen (SC) can be increased by employing a modified extraction method and hybrid of marine collagen with chitosan and hydroxyapatite using a freeze-drying and lyophilization method can also improve the thermal stability of marine collagen hybrid (Pallela et al., 2012; Subhan et al., 2015).

The low gelling temperature $\left(8-10{ }^{\circ} \mathrm{C}\right)$ of gelatin from coldwater fish makes it useful as the base for light-sensitive coatings that are important to the electronics trade (Norland, 1990). Thus, gelatin produced from cold-water fish like Alaska pollock, salmon, and unicorn leatherjacket possesses relatively lower gel strength at $10{ }^{\circ} \mathrm{C}$ (Lin et al.,2017). Cold-water fish gelatin also provides a good medium for precipitating silver halide emulsions since this process can be carried out at a lower temperature than with warmblooded animal gelatin (Norland, 1990). Furthermore, aquatic gelatins with lower gel melting temperature have desirable functional properties and better sensory characteristics (Choi and Regenstein, 2000). Generally, the properties of gelatins are determined by two main factors, namely the characteristics of the source collagen and the specific production process employed (Lin et al., 2017). For example, cold-water fish gelatin films showed significantly lower water vapor permeability (WVP) than warm-water fish and mammalian gelatin films (Avena-Bustillos et al., 2006; Lin et al., 2017).

\subsection{Proteoglycan}

Cartilaginous tissues are by-products of the seafood industry. They contain valuable glycosaminoglycans which are mainly chondroitin-4-sulfate and chondroitin-6-sulfate, present in the form of proteoglycans (Garnjanagoonchorn et al., 2007). Glycosaminoglycans originating from marine sources are different from those of terrestrial organisms, mainly in terms of their molecular weight and sulfation (Valcarcel et al.,2017). For example, marine glycosaminoglycans have distinct sulfation with different interrelated aspects such as the relative abundance of sulfated units which varies between marine and terrestrial glycosaminoglycans. The rare disaccharide units, such as chondroitin sulfate-keratan, are only found in marine sources (Higashi et al., 2015; Valcarcel et al., 2017). Chondroitin sulfate is a typical mucopolysaccharide (Guérard, 2007). Chondroitin-4-sulfate and chondroitin-6-sulfate are composed of $N$-acetyl-D-galactosamine and D-glucuronic acid sulfated at positions 4 and 6, respectively (Garnjanagoonchorn et al., 2007). Skate cartilage is reported to be exclusively composed of chondroitin sulfate (Roy, 1998) covalently attached to a core protein and embedded in a network of collagen fibrils (Carney and Muir, 1988). Chondroitin sulfate has been widely used for its chondroprotective effect (Pipitone, 1991). It could be enzymatically extracted from cartilage (Nakano et al., 1996; Lignot, et al., 2003; Garnjanagoonchorn et al., 2007). However, chondroitin sulfates from marine origin are more varied (Novoa-Carballal et al.,
2017) and those from fish processing by-products could be used as alternative sources of chondroitin sulfate. For example, heads from Prionace glauca, and skeleton from Raja clavata and a common fish by-catch small-spotted catshark (Scyliorhinus canicula) were identified as potential sources of chondroitin sulfate due to the higher yields of production and higher availability in the fish processing industry (Blanco et al., 2015; Vázquez et al., 2016).

Clinical studies on osteoarthritis patients have confirmed the therapeutic effects of orally taken chondroitin sulfate in improvement of joint function and pain reduction (Morreale and Manopulo, 1996; Bucsi and Poor, 1998; Ronca and Palmieri, 1998). Furthermore, it has been found that many therapeutic functions such as anti-angiogenic and anti-metalloproteinase activities of shark cartilage are associated with the presence of chondroitin sulfate (Guérard, 2007). Solubilization of chondroitin sulfate requires degrading both the collagen matrix and the core protein. Shark cartilage is marketed by a number of different manufacturers; branded products include Cartilade (Solgar, BioTherapies, Source Naturals), Mega Shark Cartilage (Twinlab), Sharkilage (Futurebiotics), and BeneFin Shark Cartilage (Lane Labs). Moreover, as inflammatory agents, marine originated chondroitin sulfate plays an important role. Shrimp heparin, fucosylated chondroitin sulfate from sea cucumber, and chondroitin sulfate from shark cartilage are some of the marine originated anti-inflammatory agents that show in vitro and in vivo activities (Valcarcel et al., 2017). Apart from these therapeutic activities, chondroitin sulfate also appears as the most promising compound which promotes neurite outgrowth and can be utilized for nerve regeneration (Sugahara and Mikami, 2007; Serrano et al.,2014 ) Chondroitin sulfate extracted from shark, ascidians, sea urchin, squid and octopus showed neurite outgrowth promoting characteristics (Higashi et al.,2015; Li et al., 2007);) as well as potential for nerve regeneration (Valcarcel et al., 2017).

\subsection{Surimi}

Processing the protein by-products into surimi is a popular option for increasing utilization of the resource (Martín-Sánchez et al., 2009). Surimi is a Japanese term for deboned, minced, and washed fish flesh (Figure 3) and can also be defined as a commercial preparation of fish myofibrillar protein which may serve as a main ingredient in a variety of formulated seafood products (Jannat-Alipour et al.,2019). For example, it can be used for the manufacture of seafood imitation products such as crabsticks and chunks, and flakes, among others (Martín-Sánchez et al., 2009; Kristinsson, 2007). Since commercial demand for white-fleshed fish is higher than that for other fish species, surimi is typically made from white-fleshed fish such as pollock or hake (Lee 1994; Venugopal 1997; Venugopal and Shahidi 1998). Alaska pollock is the most valuable species because it has good gelation properties, desirable odor, white color, and good cooking tolerance. Thus, it is used for the production of higher-quality surimi. Moreover, surimi is considered as a convenient intermediate product used in the preparation of ready-to-eat seafood such as kamaboko, fish sausage, imitation crab legs and shrimp products (Gao et al., 2018). Pacific whiting (Merluccius productus), as such, is also one of the main species for surimi production (Velazquez et al., 2008). However, it has been reported that surimi products can also maximize the use of less popular and underutilized fish species in order to upgrade them as human food (Alipour et al., 2018). Lizard fishes of the genus Saurida, bluefin gurnard Chelidonichthys kumu and other by-catch of trawl fisheries are some of the examples that have progressively been considered in the production of surimi (Guenneugues and Ianelli 2014; Galal-Khallaf et al., 2016). 


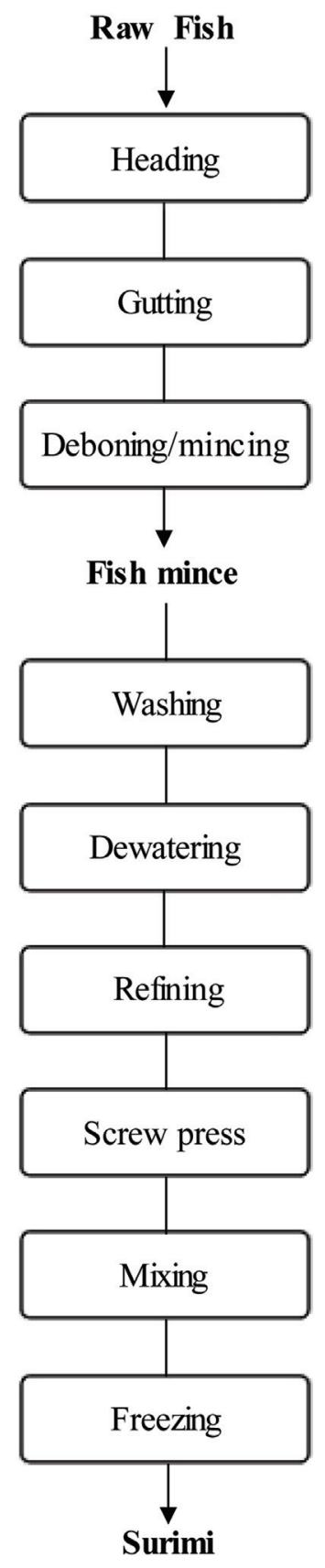

Figure 3. Processing steps in conventional surimi processing. Source: Adapted from Martin-Sanchez et al. (2009).

According to FAO (2008), the catch of pelagic fish as by-catch is increasing, whereas some of the most valuable species such as Alaska pollock (Theragra chalcogramma) are declining. Further, the demand for fish protein throughout the world is increasing faster than the available supply using traditional resources (Hultin and Kelleher 2000; Jaczynski, 2008). Hence, the manufacture of surimi can be an alternative to make use of by-catch and byproducts that are unwanted or unsuitable for other processes and to produce edible proteins from a range of other sources (Baxter and Skonberg 2008; Alvarez et al., 1992; Karayannakidis et al., 2008). However, this kind of surimi differs from conventional fish surimi in a number of characteristics (Martın-Sanchez et al., 2009). Generally, the quality of surimi products is mainly determined by gel-strength, water-holding capacity and sensory characteristics (Zhang et al., 2019). In case of cephalapodas, despite the fact that acceptable collagen-based edible gels can be obtained as reported by De La Fuente-Betancourt et al. (2009), the traditional method to make surimi cannot be applied to cephalopod muscle because its myofibrillar protein is much more water-soluble than fish muscle, with some odorous compounds, and its high protease activity that results in decreased functionality of the product so obtained (Martın-Sanchez et al., 2009).

Jonah crab (Cancer borealis), a by-catch of the lobster industry, has become popular due to increased consumer demand for crab claws due to their mild flavor and relatively low cost (Baxter and Skonberg, 2006, 2008). One concern is that while claws are the most popular part, much of the remaining crabmeat is wasted after their removal. Therefore, the low-value crab mince can be obtained from the neglected meat, a potential resource of protein for surimi-like gels, using mechanical deboning equipment (Baxter and Skonberg 2008; Galetti, 2010).

The principal purpose of catching shark is to obtain shark fin for high-class Chinese cuisine. Furthermore, the most abundant available blue sharks in Japan are landed as a secondary catch from long-line tuna fishing (Ishimura and Bailey, 2013; Nomura, 2001). After removal of the fin, shark meat is left as a by-product, which can also be used as a surimi material for making kamaboko (Nomura, 2001). However, reports about surimi from sharks and rays are scarce, but Turan and Sonmez (2007) found that thornback ray (Raja clavata) was a suitable resource for surimi production, and Kailasapathy and Salampessy (1999) made surimi from angel shark (Squatina spp.) with the addition of urease to remove the urea from shark mince without affecting its myofibrillar proteins.

\subsection{Fish protein hydrolysate and bioactive peptides}

One of the approaches for the effective protein recovery from the by-products is enzymatic hydrolysis, which is widely used to improve and upgrade the functional and nutritional properties of proteins (Je et al., 2005; Kristinsson, 2007; Kim and Mendis, 2006; Slizyte et al., 2009; Shahidi et al., 1995; Shahidi et al., 1994; Yu and Brooks, 2016). Fish protein hydrolysates have similar amino acid profiles to their original material except for the sensitive amino acids such as methionine and tryptophan that are affected to a relatively large extent during enzymatic hydrolysis (Shahidi et al., 1995). Figure 4 shows the outline of the main steps in the production of fish protein hydrolysate (FPH). Nowadays, fish protein hydrolysates are of significant interest, due to their potential application as a source of bioactive peptides in nutraceutical and pharmaceutical domains as well as in the preparation of high end pet feedstocks (Cudennec et al., 2008; Halim et al., 2016; Picot et al., 2010). One of the most important factors in producing protein hydrolysates with desired functional properties is their use in production of functional materials with desirable health effects (Deeslie and Cheryan, 1991).

Fish protein hydrolysates (FPH) have good solubility over a wide range of ionic strength and $\mathrm{pH}$ and usually tolerate high temperatures without precipitating (Skanderby, 1994). FPH have good functional properties and can contribute to water holding, texture, gelling, whipping, foaming, and emulsification properties when added to food (Kristinsson, 2007). Several reports have suggested that there is an optimum molecular size or chain length for peptides to provide good foaming and emulsifying properties, and 


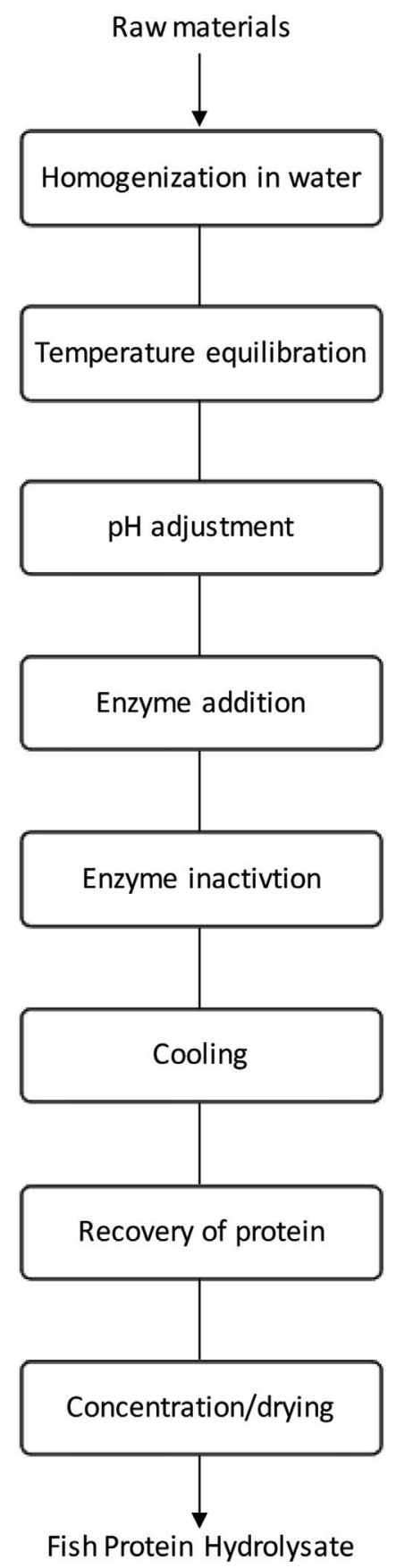

[FPH]

Figure 4. Flow diagram for the preparation of fish protein hydrolysate. Source: Adapted from Kristinsson (2007).

that limited hydrolysis resulting in larger peptides generally leads to improved emulsification and foaming properties, while extensive hydrolysis resulting in small peptides reduce these properties (Slizyte et al., 2009). In addition, except for the deficit of a few amino acids, hydrolysates have a high nutritional value (Chalamaiah et al. 2012; Halim et al., 2016; Slizyte et al., 2005; Shahidi et al., 1995). Furthermore, several studies have shown that protein hydrolysates containing mostly di- and tripeptides, are absorbed more rapidly than the free form amino acids and much more rapidly than intact proteins (Di Pasquale, 1997; Manninen, 2004).

The peptides produced from the action of enzymes (mixture or several) have proven to be good sources of antioxidants, such as capelin protein (Amarowicz and Shahidi, 1997; Najafian and Babji, 2012), mackerel protein (Morales-Medina et al., 2016; Wu et al., 2003), jumbo squid skin (Mendis et al., 2005; Suárez-Jiménez et al., 2015), hoki frame protein (Kim et al., 2007), yellowfin sole frame protein (Jun et al., 2004), yellow stripe trevally (Selaroides leptolepis) (Klompong et al., 2007), yellowfin sole skin gelatin hydrolysate (Kim et al., 1996), Alaska pollack skin gelatin hydrolysate (Kim et al., 2001), Alaska pollack frame protein hydrolysate (Je et al., 2005), and sardinelle (Sardinella aurita) by-products hydrolysate (Bougatefa et al., 2010). In these studies, peptides fractions from protein hydrolysates showed different antioxidant effectiveness. The antioxidative peptides commonly sourced from fish have been reported with molecular weights between 0.5 and $1.5 \mathrm{kDa}$ (Halim et al., 2016). According to Amarowicz and Shahidi (1997), among the four peptides isolated from the capelin protein hydrolysates, one fraction possessed a notable antioxidant activity and another two had a weak efficacy while the fourth fraction exerted a prooxidant effect in a $\beta$-carotene-linoleate model system. Meanwhile, protein hydrolysates prepared from seal meat were found to serve as phosphate alternatives in processed meat applications and reduced the cooking loss considerably (Shahidi and Synowiecki, 1991). Kim et al. (2001) prepared protein hydrolysate from Alaska pollack skin using a multienzyme system in a sequential manner. The enzymes used in the order of application were Alcalase, Pronase E, and collagenase. The fraction from the second step, which was hydrolyzed by Pronase E, was composed of peptides ranging in molecular weight from 1.5 to $4.5 \mathrm{kDa}$ and showed a high antioxidant activity. In this study, two peptides were isolated using a combination of chromatographic procedures, and these were composed of 13 and 16 amino acid residues. Both of these peptides contained a glycine residue at the C-terminus and the repeating motif Gly-Pro-Hyp. The peptide with the sequence Gly-Pro-HypGly-Pro-Hyp-Gly-Pro-Hyp-Gly-Pro-Hyp-Gly was more effective in inhibiting the formation of oxidation products (TBARS) in linoleic acid compared to the other peptide fraction with amino acid sequence Gly-Glu-Hyp-Gly-Pro-Hyp-Gly-Pro-Hyp-Gly-Pro-HypGly-Pro-Hyp-Gly. The difference in antioxidant activity between the two peptide isolates was thought to be attributed to the additional three amino acid residues (Gly-Glu-Hyp) at N-terminus of the latter peptide (Kim et al., 2001). He et al. (2006) demonstrated that protein hydrolysate prepared from Shrimp (Acetes chinensis) by a crude protease inhibited hydroxyl radical generation by about $42 \%$. Recently, Chi et al. (2015) extracted protein hydrolysate from heads of bluefin leatherjacket (Navodon septentrionalis) by using papain. Three antioxidant peptides were identified from this protein hydrolysate with specific sequences of Trp-Glu-Gly-Pro-Lys, Gly-Pro-Pro, and Gly-Val-Pro-Leu-Thr. They exhibited significant radical scavenging ability in DPPH, ABTS, hydroxyl radicals, and superoxide radical experiments. For more antioxidant data on fish protein by-product, the reader may refer to other reviews (Liu et al., 2016; Sila and Bougatef, 2016). Overall, high hydrolytic rate in hydrolysate preparation results in the formation of shorter and more active peptides that serve as electron donors and react with free radicals to stop chain reactions (Chi et al., 2015; Centenaro et al., 2014).

The antioxidant activity of proteins and peptides is due to specific scavenging of radicals formed during peroxidation, scavenging of oxygen-containing compounds, or chelation of prooxidant metal ions (Kristinsson, 2007). Therefore, production of fish protein hydrolysates with antioxidant properties will enable formula- 
tion of protein enriched and oxidatively stable seafood.

In addition to antioxidative effect, bioactive peptides isolated from various fish protein hydrolysates have shown antithrombotic (Rajapakse et al., 2005), immunomodulatory (Gildberg et al., 1996), antimicrobial (Zamora-Sillero et al., 2018), and antiproliferative (Umayaparvathi et al., 2014) activities. In another study, Kim et al. (2000) reported that some peptides derived from fish showed antihypertensive activity by inhibiting the action of angiotensin I converting enzyme (ACE) even stronger than that of many other natural peptides. The study of Cheung and Li-Chan (2010) demonstrated that protein hydrolysate produced from shrimp processing by-products also had ACE inhibitory activity. A more recent study by Nasri et al. (2013) found a high percentage of aliphatic amino acids such as isoleucine, leucine and methionine in goby protein hydrolysate which was positively associated with ACE inhibitory activity.

Kim et al. (1999) and Jung et al. (2005) reported that fish peptides are capable of accelerating calcium absorption. Furthermore, fish protein hydrolysates were found to possess hormone-like peptides and growth factors that accelerated calcium absorption (Fouchereau-Peron et al., 1999). Thus, recovery of fish protein from by-products may be of great value. In addition, fish protein hydrolysates commonly used as nutritional supplements (commercial names PC60 and Stabilium 200) were reported to reduce anxiety in humans and to improve memory and learning performances in animals and human patients (Dorman, et al., 1995; Landsberg et al., 2015; Le Poncin, 1996a, 1996b; Gevaert et al., 2016).

\subsection{Fish silage}

As mentioned earlier, virtually all fish contain $15-20 \%$ protein and some contain high amounts of oil. Fish silage or liquefied fish protein is a simple way to convert fish by-catch and fish processing by-products into a very nutritious feedstuff for productive and fur-bearing animals. The well- established markets for silage from fish waste can be found in Scandinavia, Poland, Denmark and the Netherlands (Mack et al., 2004). In addition, fish silage has been used extensively as a feed in aquaculture for a number of fish and shellfish with a promising potential in the field of larval nutrition (Martínez-Alvarez et al., 2015; Raghunath and Gopakumar, 2002; Rustad et al., 2011). It could be used as an ingredient in feed, supplying protein and water in the formulation to obtain the proper density and stability required for use in aquaculture farms (Gill, 2000). Silage made from whole fish or parts of fish, to which a mineral acid such as formic acid or lactic acid bacteria derived from fermentable carbohydrate substrates are added to lower the $\mathrm{pH}$ to below 4.5 (Guérard et al., 2005; Goddard and Perret, 2005; Vázquez et al., 2011). Liquefaction is carried out by endogenous enzymes naturally present in the fish (Guérard et al., 2005; Rai et al., 2011). Acid aids in accelerating the process by creating the right conditions for the enzymes to work and by helping to break down bone. This procedure efficiently prevents the growth of spoilage bacteria (Ghanbari et al., 2013; Tatterson, 1982). After liquefaction, it is convenient to remove the oil coming from the raw material and the protein in the aqueous layer may thereafter be dried or semi-dried (Goddard and Perret, 2005; Vázquez et al., 2011). The main advantages of fish silage are the recovery of fish offal and waste fish, low cost, good nutritional value of the resulting product is its long storage life. Besides, compared with fishmeal, silage generally has the advantage of no heat denaturation and the proteins are more available for the aquaculture species. The main inconvenience is the impossibility of regulating the degree of hydrolysis achieved
(Guérard et al., 2005).

\section{Marine lipid}

\subsection{Marine fish oils}

Fish oil is mainly derived from by-catch and by-products of fisheries (Rustad et al., 2011; Ambigaipalan and Shahidi, 2017; Shahidi and Ambigaipalan, 2015). Pelagic fish species, namely menhaden, capelin, herring, blue whiting, Norway pout, sand eel, sprat, sardine, jack mackerel, and anchovy, among others, caught in large quantities as by-catch are primarily used for the fish meal industry with oil being a by-product (Bimbo 2009; Shahidi 2007; Ziegler et al., 2016). A number of fish species including catfish, tuna, salmon, sardine/pilchard, white fish sp., dogfish, pollock, horse mackerel, Atlantic herring, mackerel $s p$., and hoki, among others, are caught or raised principally for food use and their by-products from gutting, filleting and other processing operations are then converted into fishmeal and oil or other products (Rustad et al., 2011; Shahidi, 2007).

In addition to being a major source of energy, marine oils are rich in polyunsaturated fatty acids (PUFA), especially the n-3 PUFA family of alpha-linolenic acid (C18:3n-3) and its elongated and desaturated products such as eicosapentaenoic acid (EPA, 20:5n-3), docosapentaenoic acid (DPA, 22:5n-3) and docosahexaenoic acid (DHA, 22:6n-3) which endows marine products especially oil-rich products the prevention capacity of various diseases such as blood pressure, coronary heart disease, inflammation, cancer and helps consumers in maintaining health (Brunborg et al., 2006; Kerry and Murphy, 2007; Sargent et al., 1989, 1999; Shahidi, 2007; Abraha et al., 2018). Besides fish oil, shellfish also has favorable lipid composition. As Phleger et al. (1998) reported, the lipid content in krill varies due to their specific diet, which could be either phytoplankton or zooplankton (Araujo et al., 2014). The Euphausia superba collected in the Elephant Island area of the Antarctic Peninsula contains $0.2 \%$ wax ester, $33.4 \%$ triacylglycerols (TAG), $12.8 \%$ free fatty acids (FFA), $2.2 \%$ sterol and $51.3 \%$ phospholipids (PL). However, a different lipid content was observed from male E. superba collected in Eastern Antarctica, with $0 \%$ wax ester, 1.7\% TAG, 0\% FFA, 5.4\% sterol, and 93.1\% PL. Similarly, Gigliotti et al. (2011) showed that the extracted oil from Antarctic krill (Euphausia superba) contained predominantly phospholipids (20 33\%), polar non-phospholipids (64 77\%), and minor amount of TAGs (1 3\%). Within phospholipids, there was a total of $47.4 \%$ n-3 fatty acids consisting of 18.0\% DHA, 28.2\% EPA, and 23.5\% saturated fatty acids. On the contrary, TAG contained significantly less amount of total n-3 (4.0\%), DHA (1.1\%), and EPA (2.3\%), but more saturated FA $(38.7 \%)$. On the other hand, marine fish oil from by-products showed a better market potential, particularly those prepared by molecular distillation and enzymatic processes as well as other specially developed methods in purification of the final products. Based on the recent market report, the increase of marine fish oil consumption and their price have been more dramatic than the case of fishmeal due to the awareness of heart function as well as brain, eye, and joint health, and for prenatal development through ingestion of fish oil products (Olsen et al., 2014; Ramírez, 2013). Figure 5 shows the structures of omega- 3 fatty acids in marine oils.

Many studies have reported the fatty acid composition of fish by-products. Tuna is found to be a particularly good source of DHA-rich oil and it is derived as a by-product from canning operations (Chantachum et al., 2000). The tuna oil supplements general- 


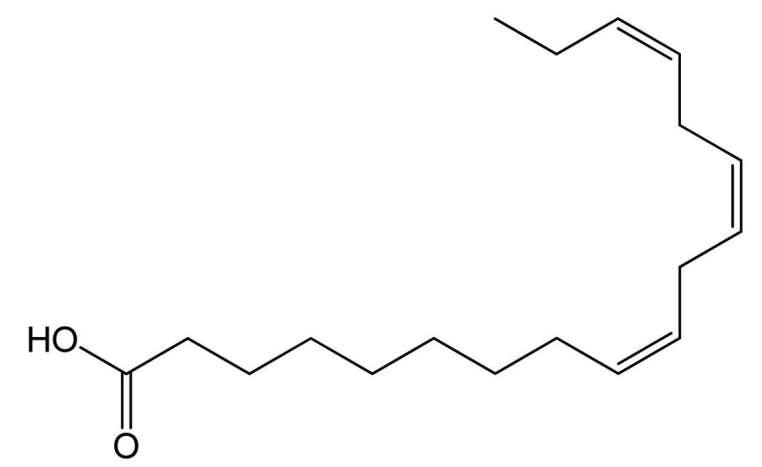

Alpha-linolenic acid (18:3n-3)<smiles>CC/C=C\C/C=C\C/C=C\C/C=C\C/C=C\CCCCCC(=O)O</smiles>

Eicosapentaenoic acid (20:5n-3)

Docosapentaenoic acid (22:5n-3)<smiles>CC/C=C\C/C=C\C/C=C\C/C=C\C/C=C\C/C=C\CCC(=O)O</smiles>

Docosahexaenoic acid (22:6n-3)

Figure 5. Structure of omega-3 fatty acid. Source: Adapted from Shahidi (2007).

ly contain higher levels of DHA than other supplements currently available (Mann et al., 2010; Nichols et al., 1998). Catfish viscera, a by-product of catfish processing, are industrially used to produce edible oils (Shahidi, 2007). According to Sathivel et al. (2003), the total unsaturated fatty acids in the purified oil from the catfish viscera was $67.7 \%$ and the combined omega-3 fatty acids was only $4.6 \mathrm{mg} / \mathrm{g}$ of oil. Significant differences have been reported in the fatty acid content and composition of salmon by-products (Shahidi 2007). Aursand et al. (1994) reported that EPA and DHA levels were higher in liver than in edible belly flap region of Atlantic salmon. Furthermore, pink salmon heads had the highest lipid content while viscera had the lowest (Wanasundara et al., 1998).

Herring oil is produced from different types of by-products, but only heads, mixed and headless by-products are of interest (Sathivel et al., 2003). In general, monounsaturated fatty acids are the major constituent class of fatty acids present in herring by-products, with docosenoic acid (C22:1) and eicosenoic acid (C20:1) being the most important among them (Aidos et al., 2003). However, no significant differences were found between the fatty acid composition of the mixed and the headless by-products or their correspond- ing oils (Aidos et al., 2002).

\subsection{Marine mammal oils}

Marine mammals are unique in that their lipids are mainly stored as subcutaneous fat, known as blubber (Holmer, 1989; Shahidi and Ambigaipalan, 2015). Blubber functions primarily as an energy source, body stream-liner, insulator and buoyancy adjuster during aerobic dives (Brunborg et al., 2006; Davis, 2014). The oils from marine mammals contain various lipid classes, including triacylglycerols (TAG), diacylglycerols (DAG), monoacylglycerols (MAG), free fatty acids (FFA), phospholipids (PL), wax esters, cholesterol, cholesterol esters, hydrocarbons, vitamins, and ether lipids (Shahidi, 2007; Beppu et al., 2017). Furthermore, fatty acid composition of marine mammal lipids varies significantly, but all contain a large proportion of long-chain highly unsaturated fatty acids (LC-HUFA), similar to fish oils (Shahidi, 1998; Shahidi and Ambigaipalan, 2015). Although EPA and DHA are found abundantly in different marine oils, DPA is present in significant 


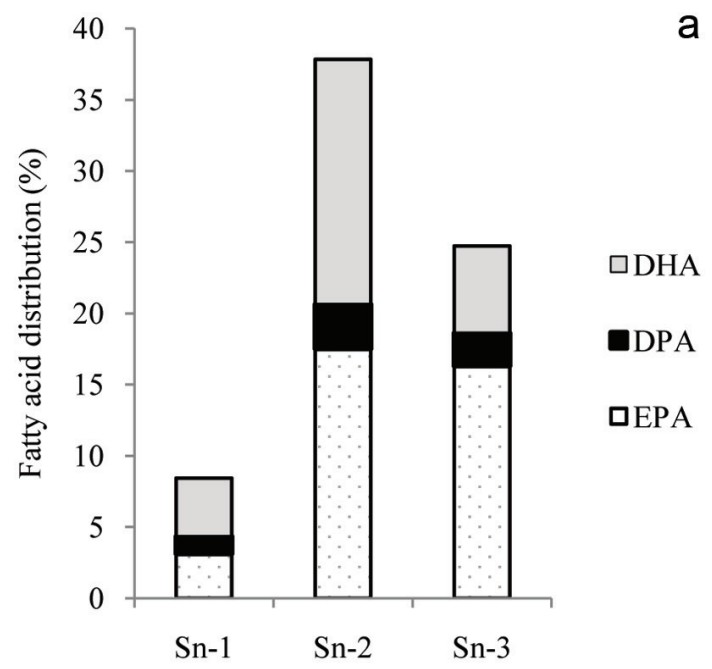

Positions of triacyglycerols

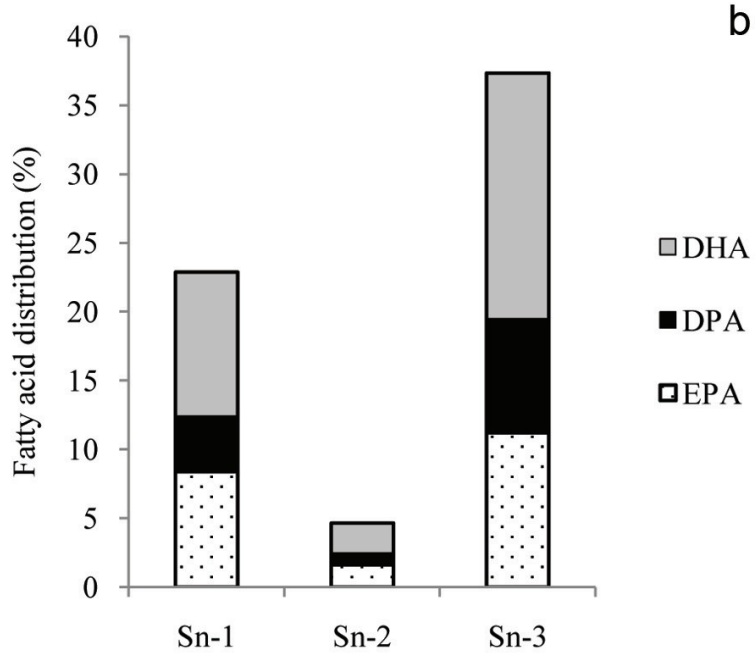

Positions of triacylglycerols

Figure 6. Fatty acid distribution (\%) in different positions of triacylglycerols of (a) menhaden oil and (b) harp seal blubber oil. Source: Adapted from Shahidi (1998) and Ackman et al. (1972).

amounts only in seal blubber oil (Broadwater et al., 2013; Wanasundara et al., 1998; Aidos et al., 2002; Sathivel et al., 2003; Jayasinghe et al., 2003). The ratio of DHA to EPA and presence of DPA in modest amount are most important (Shahidi, 2002).

The main components of seals are carcass $(44 \%)$, blubber $(29 \%)$, viscera (18\%) and skin (8\%) (Shahidi, 1998). Seal blubber and skin are widely used, but the utilization of blubber and meat for human food consumption is limited (Brunborg et al., 2006). Seal blubber oil is an important by-product of the sealing industry that is almost entirely triacylglycerols (Shahidi and Wanasundara, 1994) and is quite low in cholesterol (Schreiner et al., 2004). Many studies have shown that seal blubber is an excellent source of long chain and very long-chain $\omega 3$ polyunsaturated fatty acids (Broadwater et al., 2013; Brunborg et al., 2006; Wanasundara and Shahidi, 1998). Furthermore, the positional distribution of fatty acids on the glycerol backbone of triacylglycerols of seal blubber is fundamentally different from that of fish oil (Ackman and Ratnayake, 1989; Shahidi et al., 1996; Shahidi, 1998). While most of the PUFA of fish oil are located in the sn-2 position, they are found primarily in the sn- 1 and sn- 3 positions in marine mammal oils such as the seal blubber oil (Figure 6). The positional distribution of $\omega-3$ fatty acids in the TAG molecules of seal blubber oil is considered as being favorable because during digestion the fatty acids from sn- 1 and sn-3 positions of the TAG are liberated by position specific enzymes such as pancreatic lipase, while the fatty acids attached to the sn-2 position of the TAG are distributed in the body in the chylomicron form (Yang et al., 1990), these polyunsaturated fatty acids are in a liquid form within blood with low viscosity and high fluidity, which is believed to be a reason for the low incidence of cardiovascular disease among the Inuit of Greenland compared to that of the Danish population there, despite their high fat consumption (Ackman and Ratnayake, 1989; Bang et al., 1976; Jeppesen et al., 2014).

Since marine oils are complex mixtures of fatty acids with varying chain lengths and degrees of unsaturation, separation of individual fatty acids is difficult for production of highly concentrated individual $\omega 3$ components. However, this challenge may be overcome using a number of techniques such as adsorption chromatography, fractional or molecular distillation, enzymatic split- ting, low-temperature crystallization, supercritical fluid extraction, liquid-liquid extraction and urea complexation (Ratnayake et al., 1988; Schlenk and Holman, 1950; Shahidi et al., 1994; Shahidi and Wanasundara, 1998; Swern, 1964; Wanasundara and Shahidi, 1997, 1999). However, each of these methods has its own drawbacks and thus far only a few are suitable for large-scale production (Shahidi and Wanasundara, 1998). According to Shahidi and Wanasundara (1998), there is a growing industrial interest in the use of enzymatic methods due to the potential benefits of obtaining concentrated omega-3 oils in the acylglycerol form. A promising new $\mathrm{n}-3$ LC-PUFA extraction technique called the $\mathrm{pH}$-shift method may prove beneficial in the industry because no heat is required, thereby limiting decomposition reactions and oxidative damage that normally occurs during extraction (Okada and Morrissey, 2007).

\subsection{Application of fish and seal blubber oils}

The market for omega-3 fatty acids as supplements and nutraceuticals in Europe and Japan has long been established, and the US market is experiencing rapid growth (Olsen et al., 2014; Rasmussen and Morrissey, 2007). In general, marine oils are used in a variety of applications that include industrial, food, feed, aquaculture, and nutraceuticals use (Bimbo, 2009; Shahidi and Ambigaipalan, 2015).

\subsubsection{Application in food}

Fish oil may be used edible purposes and has historically been used in partially hydrogenated form in Europe in the baking industry. However, a wide variety of foods, including bread, baby food/ infant formula, maternal beverages, margarine and salad dressings containing marine oils have been prepared (Bimbo, 2009; Ferraro et al., 2010; Garcia-Carreno et al., 1994; Kadam and Prabhasankar, 2010; Shahidi, 2012; Suleria et al., 2015). Many of these marine oils, including fish oils have been marketed as omega-3 concentrates and have been GRAS approved by FDA, provided that the 
Table 2. Nutraceutical potentials of omega-3 fatty acids

\begin{tabular}{|c|c|}
\hline Health benefits & Reference \\
\hline Prevention of coronary artery disease & Schacky (2000) \\
\hline Protection against arrhythmias & Christensen et al. (1997) \\
\hline Reduce blood pressure & Appel et al. (1993) \\
\hline Beneficial for diabetic patients & Sheehan et al. (1997) \\
\hline Fight against manic-depressive illness & Severus et al. (1999) \\
\hline Reduce symptoms in asthma patients & Broughton et al. (1997) \\
\hline Protection against chronic obstructive pulmonary diseases & Shahar et al. (1994) \\
\hline Alleviate symptoms of cystic fibrosis & Lawrence and Sorrell (1993) \\
\hline Improve the survival of cancer patients & Gogos et al. (1998) \\
\hline Prevent relapses in patients with Crohn's disease & Belluzzi et al. (1996) \\
\hline Prevent autoimmune disorders & Kelley (2001) \\
\hline Influence on mental health and psychiatric illness & Sinn and Howe (2008) \\
\hline
\end{tabular}

combined intake of DHA and EPA does not exceed $3 \mathrm{~g} /$ person/day. Hoffmann La Roche company produces microencapsulated fish oil under the brand name of ROPUFA' for use in health food formulations. Such powdered products are used mainly in dry foods (e.g. bakery products and milk powders), low-fat products, milk-based drinks, orange drinks, salad dressings, soups, cakes and biscuits (Beindorff and Zuidam, 2010; Newton, 1996).

Barlow and Young (1988) reported novel uses of marine oils and suggested that the optimum level of their incorporation into meat products (e.g. salami, frankfurters) was 9 to $16.5 \%$ of the total lipid content. Incorporation of marine oils in highly flavored foods is preferred since any off-flavor development from the use of marine oils is masked. The refined and bleached seal blubber oil (SBO)/canola oil blends containing 0, 25, 50 and 100\% (w/w) SBO were used to prepare French salad dressings and mayonnaise without any added antioxidants. These products were stable for 2-4 weeks at room temperature without changes in their physical appearance or taste (Shahidi 1994). Ghorbanzade et al. (2017) used nano-liposome technology to nano-encapsulate fish oil which was utilized in fortifying yogurt. Through measuring various physicochemical properties such as $\mathrm{pH}$, acidity, syneresis, fatty acid composition, peroxide value as well as sensory tests of the yogurt so produced, they found that during three weeks storage at $4{ }^{\circ} \mathrm{C}$, addition of nano-encapsulated fish oil into yogurt provided longer shelf life in terms of sensory characteristics than yogurt fortified with free, unencapsulated fish oil. In an earlier study, Jafar et al. (1994) showed that mayonnaise prepared from menhaden oil had a shelf life of one day without any added antioxidants. However, added citric acid or sodium citrate and propyl gallate in the oil phase and ethylenediaminetetraacetic acid (EDTA) and ascorbic acid in the aqueous phase increased the shelf life of the product to an average of 49 days at room temperature. In addition to the direct use of marine oils in foods, concentrates or nanoliposome of EPA and DHA may be applied as PUFA supplements in a number of dairy products, mayonnaise, margarine, other table spreads, edible vegetable oils, emulsified or non-emulsified shortenings, hard fats, pastry fats, biscuits, icings and bread doughs (Bimbo, 2009; Rasti et al., 2017). A large proportion of marine oils used in food applications are hydrogenated to various degrees. Modem hydrogenation processes of high selectivity make it possible to produce a high proportion of mono- and polyunsaturates without increasing the level of saturated fatty acids, and the degree of hydrogenation can be controlled to produce oils with a wide range of physical and chemical properties in order to meet various application demands (Bimbo, 2009). However, concern about production of trans fatty acids may overshadow the use of such oils, if not fully hydrogenated.

\subsubsection{Application in nutraceuticals}

Marine oil is an excellent source of omega-3 fatty acids which are linked to the promotion of human health and to fight against numerous diseases (Shahidi, 2007; Shahidi and Ambigaipalan, 2015). Table 2 shows the nutraceutical potential of omega-3 fatty acids. Furthermore, several studies have shown the importance of n-3 and n-6 fatty acids supplementation to pregnant and lactating women (Coletta et al., 2010; Helland et al., 2001; Jensen, 2006; Mitmesser and Jensen, 2007; Swanson et al., 2012). The n-3 fatty acids, especially long-chain $n-3$ polyunsaturated fatty acids DHA and EPA exert important effects on eicosanoid metabolism, membrane fluidity, myelination, neurotransmission, ion channel, enzyme regulation and gene expression and therefore are biologically important nutrients (Calder, 2018; Calder, 2014; Jensen, 2006; Sinn and Howe, 2008; Swanson et al., 2012). Because of the biological activities of $n-3$ fatty acids, some investigators have hypothesized that maternal n-3 fatty acid intakes might have significant effects on several pregnancy outcomes, including duration of gestation and infant size at birth, preeclampsia, depression, cognition, and immunologic function (Jensen, 2006; Swanson et al., 2012). In addition, because DHA is present in high concentrations in the brain and retina, particularly in synaptic membranes and rod-cone outer membranes, adequate provision of DHA is thought to be essential for optimal visual and neurologic development during early life (Jensen, 2006; Swanson et al., 2012). Thus, maternal DHA supplementation may also affect infant visual function and neural development (Jensen, 2006; Swanson et al., 2012).

As discussed earlier, fish oil, particularly tuna oil, is a rich source of DHA. The use of fish oil supplements has gained considerable interest in a variety of applications, including additive in infant formulas. It has been reported that a deficiency of DHA exists in erythrocyte lipid, phosphatidylcholine and phosphatidylethanolamine in unsupplemented bottle-feeding compared to breastfed infants (Putnam et al., 1982; Simopoulos, 1989). DHA supple- 
mented from fish oil source formula-fed infants exhibited a more rapid rate of development of visual acuity compared to the control formula fed babies (Campoy et al., 2012; Tai et al., 2013; Uauy, 1990: Uauy, et al., 1990). However, the investigation of Nichols et al., 1998 showed that arachidonic acid (AA): EPA: DHA ratio $(0.2: 2.1: 1.0)$ in Northern hemisphere fish oil differs markedly from breast milk (2:0.2:1). Due to the presence of a high level of EPA in fish oil, which may inhibit the infant's own endogenous AA synthesis, fish oil supplemented infant formula milk may require AA co-supplementation (Hadley et al., 2017; Uauy et al., 1990). However, some analyses of flesh from selected Australian fish showed more similar ratios of these three essential fatty acids as observed in breast milk (Nicholos et al., 1998).

Among marine oils, cod liver oil is dominant in the market; it is rich in n-3 PUFA as well as containing high levels of vitamins A and D (Shahidi, 2007; Engelhardt and Walker, 1974). Similar to cod liver oil, halibut liver oil is also used as prime source of vitamins $\mathrm{A}$ and $\mathrm{D}$, as both of these fish species belong to the lean white fish category (Shahidi, 2007).

Fish oils and their $\omega-3$ fatty acid concentrates have also been used in pharmaceuticals such as tendon, skin and hair care products and EPA and DHA capsules due to their promoting effect on collagen synthesis. Chan et al. (2016) reported the positive healing effect of therapeutic ultrasound coupling with topical fish oil in treating soft tissue (Achilles tendon) injuries. Commercially, seal blubber oil (SBO) products are mostly prepared as nutritional supplements in the form of gelatin capsules (Bimbo 2009). Osterud et al. (1995) have reported that supplementation of a regular diet with a combination of SBO and cod liver oil would have beneficial effects with respect to cardiovascular and thrombotic diseases. Due to its high content of long-chain PUFA, SBO may provide an alternative starting material for the production of $\omega 3$ fatty acid concentrates.

\subsubsection{Application in animal feed formulations}

\section{Designer Eggs}

The consumption of eggs has been decreasing because of recommendations by health professionals to reduce dietary cholesterol. Eggs with modified fat content have become very popular and are available in many parts of the world. This involved the production of low cholesterol, high omega-3 fatty acid eggs now called designer eggs. However, the key aspect of designer eggs is the synergistic combination of n-3 fatty acids with major antioxidants, lutein and vitamin E (Rajasekaran and Kalaivani, 2013; Surai et al., 2001; Zaheer, 2015). The aim of such production is to obtain eggs with omega- 6 to omega- 3 fatty acid contents are at the optimal 1:1 ratio. One large egg obtained in this way can supply more than $600 \mathrm{mg}$ of total omega-3 fatty acids with a significantly elevated polyunsaturates to saturates ratio of 0.6 to 1.02 and lowering the omega-6:omega-3 fatty acid ratio from $10: 1$ to $1: 1$, which is able to satisfy the recommended regular fish consumption of WHO (1-2 servings per week, equivalent to 200 to $500 \mathrm{mg}$ /day EPA + DHA) as protective against coronary heart disease and ischaemic stroke. (Lesnierowski and Stangierski, 2018; Watson, 2002). While the cholesterol issue remains controversial, feeding $1.5-3 \%$ fish oil in layer hen diets will increase the $\omega 3$ levels in the egg yolk. Flavor is not affected until levels of $6 \%$ are used (Bimbo 2009). Shimizu et al. (2001) demonstrated that dietary LCn-3PUFA accumulate preferably in the phospholipid (PL) fraction of the egg by efficiently replacing AA, even after moderate supplementation of $n-3 F A$. This mechanism might possibly explain why, when fish oils are fed in excess of about $1.5 \%$ in the diet, the amount of LCn-3PUFA in the egg is not altered to any great extent (Schreiner et al., 2004).

Broiler

Infectious diseases cause serious economic losses to the poultry industry every year. Dietary lipids are one class of nutrients that offer tremendous opportunity for modifying immune responses in addition to supplying energy in animals (He et al., 2007; Hwang, 2000; Kouba and Mourot, 2011; Miles and Calder, 1998; Calder et al., 2002 and 2014). Fritsche et al. (1991) have reported that feeding chickens a diet rich in $\omega 3$ PUFAs ( $70 \mathrm{~g}$ fish oil $/ \mathrm{kg}$ of diet) significantly enhances their primary antibody response and alters lymphocyte proliferation. Research indicates that prostaglandin E2 is immunosuppressive and that the $\omega 3$ fatty acids in menhaden oil reduce its biosynthesis (Liu and Denbow 2001; Moison and Henegouwen, 2001). Fish oil also manipulates the fat composition of broiler meat (Hargis and Elswyk, 1993). The study of Konieczka et al. (2017) shows that one week of feeding fish-oil diets $(10 \mathrm{~g} / \mathrm{kg}$ diet) to 15-day-old female broilers Ross 308 was sufficient to enrich breast meat with LC-PUFA, whereas two weeks were needed to effectively enrich thigh meat with these FA. A $100 \mathrm{~g}$ portion of such breast or thigh meat would provide on average $33 \%$ and $15.5 \%$, respectively, of the recommended daily intake of EPA and DHA for humans, and therefore make enriched chicken meat a superior source of LC-PUFA than lean fish meat.

\section{Ruminant}

Because of the bad publicity about red meats, several researchers have been attempting to decrease the saturated fatty acids and incorporate omega-3 fatty acids into the flesh of red meat animals. Recently, there has been a great deal of interest in feeding fat to dairy cows in order to increase nutrient density of the diet and improve reproduction (Petit-Omega-dsm.com). In lactating dairy cows, it was reported that a product rich in omega-3 fatty acids sourced from fish oil played a role in reducing prostaglandin production which plays an important part in embryo implantation by preventing embryonic loss (Thatcher and Staples, 2000; PetitOmega-dsm.com). Meanwhile, modification of the diet of cows with fish oil significantly increased the concentration of bioactive whey proteins, fat-soluble vitamins (E, A), and carotene ( $\alpha$-retinol) with $\alpha$-tocopheroll, as well as EPA and DHA ratio in fatty acid composition of the milk. Therefore, introducing fish oil improved the antioxidant properties of milk at the same time (Puppel et al., 2016). Furthermore, the findings of Burke et al. (1997) showed that feeding lactating dairy cows of low fertility a source of EPA and DHA in menhaden fishmeal increased pregnancy rates from 31.9 to $41.3 \%$.

\section{Aquaculture}

The marine food chain consists of plankton, algae and other fish which offers some unique ways to increase $\omega 3$ 's in the diet by the feeding of fish. Therefore, lipids from wild fish particularly marine fish contain comparatively high levels of $\omega 3$ fatty acids (Shahidi 2007; Yongmanitchai and Ward 1989) compared to many farm-raised fish. This deficiency can be eliminated by adding fish oil containing high levels of $\omega 3$ to their diet. The composition of shrimp, crayfish, catfish, eel, trout and carp have been reviewed 

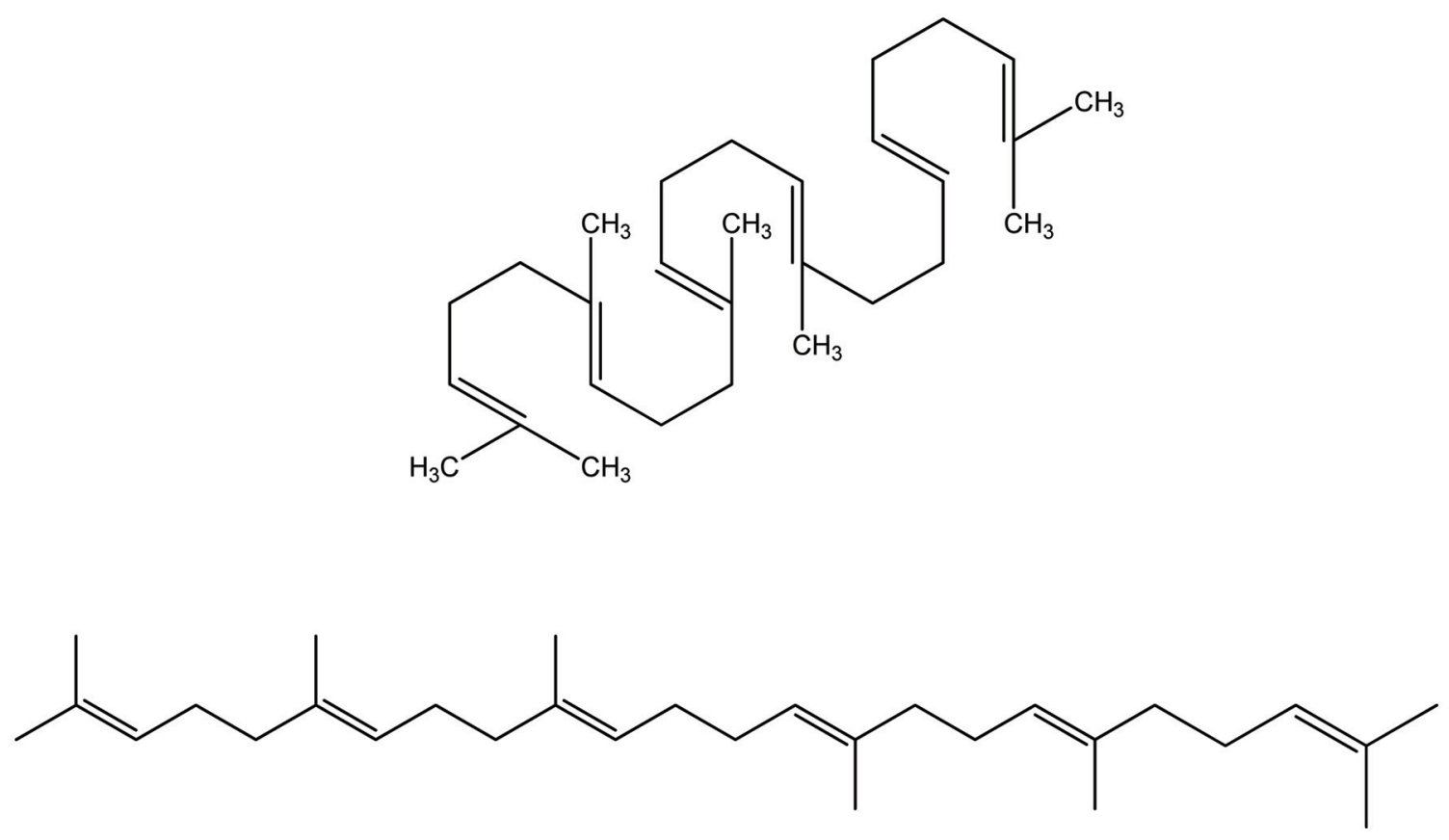

Figure 7. Chemical structure of squalene.

and it is apparent that $\omega 3$ 's are going to have an effect on the future of all commercial aquaculture (Bimbo 2009). However, to meet the increased demand for seafood products due to population increase and to control cost, a greater proportion of fish consumed would be farmed and with using terrestrial fats. From the report of Sprague et al. (2016), by constantly comparing the fatty acid composition of over 3,000 farmed Atlantic salmon for 10 years, in 2015, the $n-3$ fatty acid in flesh decreased to only $~ 50 \%$ of that in 2006 due to the tendency of replacement of marine oil by terrestrial fat in their feedstock. "Consequently, the nutritional value of the final product is compromised requiring double portion sizes, as compared to 2006, in order to satisfy recommended EPA+DHA intake levels endorsed by health advisory organisations" (Sprague et al., 2016).

\subsection{Squalene}

Squalene $\left(\mathrm{C}_{30} \mathrm{H}_{50}\right)$, a shark by product, is a highly unsaturated thermally unstable and light-sensitive triterpenoid aliphatic hydrocarbon with six unconjugated double bonds (Figure 7). It occurs in high levels in livers of certain deep-sea sharks, although plant sources are now used as well, such as rice bran, wheat germ, amaranth, pumpkin seeds, lotus pollen and olives (Blumer, 1967; Deprez et al., 1990; Hilditch and Williams, 1964; Nergiz and Çelikkale, 2011; Naziri et al., 2016; Pokkanta et al., 2019; Summers, 1987; Tsujimoto, 1932; Xu et al., 2011).

In the case of shark liver oil, squalene is a major component comprising up to $89 \%$ of the oil (Peyronel et al., 1984). In addition, shark liver oil serves as an excellent source of vitamin A and other bioactive compounds (Shahidi, 2007). Squalene was first discovered in shark liver oil in 1906 by a Japanese marine oil chemist, Mitsumaru Tsujimoto. Squalene is a metabolic precursor of cholesterol and other sterols. In humans, over $60 \%$ of ingested squalene is absorbed from the small intestine; from there it is carried in the lymph in the form of chylomicrons into the systemic circulation. In the blood, squalene is carried mainly in very-lowdensity lipoproteins and distributed to various tissues of the body with a large percentage being distributed to the skin. Therefore, the increase in cholesterol synthesis is not associated with consistent increase in serum cholesterol level (Boskou, 2008).

In a model study using methyl oleate and methyl linoleate as the substrates, Govind Rao and Achaya (1968) demonstrated that squalene has antioxidant activity. Boskou (1998) reported that squalene retarded the degradation of unsaturated fatty acids at high temperatures. The application of squalene for skin care has been studied for atopic dermatitis patients (Tanii et al., 1991). In body care products, squalene is used as an ingredient, which acts as an effective moisturizer, wrinkle remover and wound healer. This hydrocarbon appears to function on the surface of the skin as an antioxidant, capable of quenching singlet oxygen and protecting the skin from damage due to exposure to UV and other sources of ionizing radiation. Thus, squalene from shark liver is now sold as a high-grade cosmetic (Kaiya, 1990; Shinposya, 1989). Chemopreventive effect of squalene against certain cancers has been reported in animal models (Rao et al., 1998; Smith et al., 1998; Newmark, 1999), but a later investigation by Scolastici et al. (2004) questioned the chemopreventive effect of squalene. However, squalene supplements are considered a promising anti-cancer, anti-radiatory, and anti-oxidative agent, although human trials have yet to be performed that could verify its usefulness in cancer therapy (Boskou, 2008; Reddy and Couvreur, 2009). Squalene has also been used as an emulsifier in certain adjuvant formulations of seasonal influenza virus vaccine given to patients since 1997 though there was suspicion of incidence of anti-squalene antibodies in soldiers using anthrax vaccine during Gulf War, nonetheless, it was later verified to be unrelated with squalene injection (Del Giudice et al., 2006; Lippi et al., 2010). Nowadays, squalene is still used as an immunologic adjuvant, which is substance, administered in conjunction with a vaccine that stimulates the immune system and increases response to the vaccine (WHO 2008).

Squalane $\left(\mathrm{C}_{30} \mathrm{H}_{62}\right)$, an oily fully saturated material, is obtained 


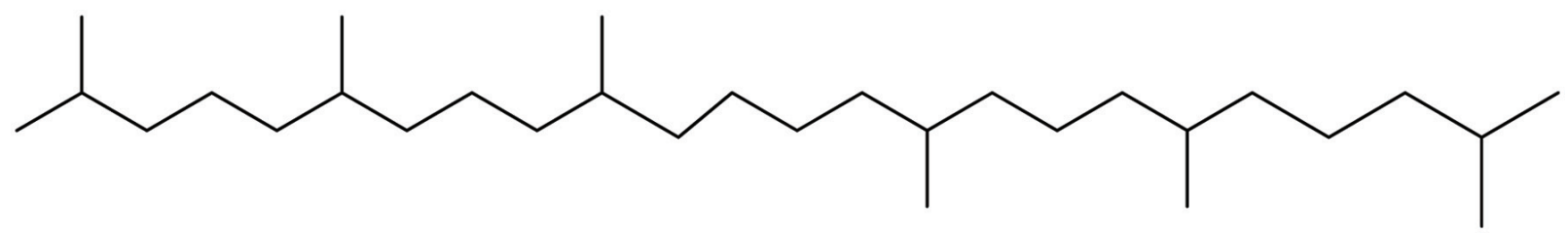

Figure 8. Chemical structure of squalane.

by catalytic hydrogenation of squalene (Figure 8) (Takamatsu, 1994). It is used as a skin lubricant and emollient in foundation creams, lipsticks, and hair lotions (Kaiya 1990).

\section{Chitin, chitosan and their oligomers and monomers}

\subsection{Chitin and chitosan}

Chitin is a major component of the exoskeleton of invertebrates, crustaceans, insects and the cell wall of fungi and yeast and acts as a supportive and protective component (Cohen, 2001; Hayes et al., 2008a; Knorr, 1991; Shahidi et al., 1999). Chitin is the second most abundant high molecular weight natural biopolymer on earth after cellulose, with an annual production of approximately $10^{10}-10^{11}$ tons by living organisms (Revathi et al., 2012; Hamed et al., 2016). Moreover, chitin is an odorless/tasteless nitrogenous polysaccharide which in its pure state is white or off-white in color (Cheba, 2011). It is composed of $\mathrm{N}$-acetyl- $\beta$-D-glucosamine units and its monomers are attached via $\beta(1 \rightarrow 4)$ linkages, which is similar to cellulose except that secondary hydroxyl groups on the alpha carbon atom of the cellulose molecule are substituted with acetamide groups (Figure 9) (Shahidi, 2007; Shahidi et al., 1999; White et al., 1968). Chitosan is derived from varying degrees of deacetylation of chitin. Therefore, chitosan is a copolymer consisting of $\beta$ - $(1 \rightarrow 4)$-2-acetamido-D-glucose and $\beta$ - $(1 \rightarrow 4)$ 2 -amino-D-glucose units with the latter exceeding $80 \%$ (Shahidi et al., 1999). These reactive amino groups may participate in the formation of a large variety of useful derivatives that are commercially available or can be made available via graft reactions and ionic interactions (Dutta et al., 2004). The degree of acetylation and degree of polymerization are the two most important structural parameters which dictate the use of chitosan for various applications (Agboh and Qin, 1996; Mourya and Inamdar, 2008; Shahidi 2007). According to Shahidi et al. (1999), most physiological activities and functional properties of chitin and chitosan oligomers clearly depend upon their molecular weights; a chain length of at least five residues is required. Chitin is insoluble in water and most organic solvents, but may be dissolved in a few solvents including hexafluoroisopropanol, hexafluoroacetone, and chloroalcohols in conjunction with aqueous solutions of mineral acids, and dimethylacetamide (DMAc) containing 5\% lithium chloride (LiCl) (Dutta et al., 2004). However, chitosan dissolves in nearly all aqueous acids, of which the most commonly used are formic acid and acetic acid. Its solubility, in methyl morpholine- $N$-oxide (NMMO) $/ \mathrm{H}_{2} \mathrm{O}$ has also been reported (Dutta et al., 2004; Shahidi, 2007; Mourya and Inamdar, 2008).

Shellfish waste consisting of crustacean exoskeletons is currently the main source of biomass for chitin production (Shahidi et al., 1999; Agullo et al., 2003). As already mentioned, 50-80\% of whole shellfish ends up as by-product and this creates a practical challenge and environmental hazard concern exist due to irregular dumping of this waste in-land and in the oceans (Hamed et al., 2016). Therefore, the extraction of chitin from crustaceans and its value-added processing maybe a way to minimize the concern while leading to the production of valuable compounds with remarkable biological properties. Crustacean shell consists primarily of protein (20-40\%), mineral salts (mainly carbonate and phosphate, 30-60\%) and chitin (13-42\%) along with others compounds such as carotenoid pigments (e.g., astaxanthin) and lipids (Tharanathan, 2002; Agullo et al., 2003; Vani and Stanley, 2013). These proportions vary with species and season (No and Meyers, 1997). Out of the different species of crustaceans, crab and shrimp shell wastes are the most exploited sources of chitin (Muzzarelli, 1977; Johnson and Peniston, 1982; Tweedie et al., 2004; Stepnowski, et al., 2004; Armenta-Lopez et al., 2002; Charoenvuttitham et al., 2006). According to Shahidi and Synowiecki (1991), on a dry weight basis 17.0 to $32.2 \%$ chitin is present in shrimp and crab section by-products.

Chitin and chitosan are also known to exhibit polymorphism and the polymorphic forms of chitin differ in the packing and polarities of adjacent chains in successive sheets. The chains can be arranged in microcrystalline structures of antiparallel sheets $(\alpha$-chitin), parallel sheets $(\beta$-chitin) and a combination of both $(\gamma$-chitin) (Figure 10) (Ramakrishnan and Prasad, 1972; Tharanathan, 2002; Kurita, 2001). However, the most common and stable allomorph of chitin exists in $\alpha$-conformation which is a resistant structure and is present in insect cuticles, sponges, shells of crabs, lobsters and shrimp, as well as fungal and yeast cell walls (Hamed et al., 2016; Mourya and Inamdar, 2008). Squid pen and extracellular fibers of diatoms are important sources of the polymorph $\beta$ chitin (Kurita et al., 1993).

Both chitin and chitosan find extensive applications in medicine and pharmacology, agriculture, food, biotechnology, nutraceuticals, bioremediation, gene therapy and cosmetics due to their unique chemical characteristics such as biodegradability, nontoxic, biocompatibility, physiological inertness and hydrophilicity (Shahidi, 2007; Hayes et al., 2008b; Agullo et al., 2003; Jeon et al., 2000; Shahidi et al., 1999). However, chitin has a limited application compared to chitosan because of its poor solubility and reactivity (Park et al., 2004). Schematic representations of chitin and chitosan manufacture by industrial methods are shown in Figure 11. Chitin biodegradability is due to chitinases that are widely distributed in nature and also found in bacteria, fungi and plants and in the digestive tract of numerous animals (Rinaudo, 2007). Furthermore, chitosan is metabolized by certain human enzymes, especially lysozyme (Prabaharan et al., 2007). It was shown that the rate of in vivo degradation is high for chitin but decreases when the degree of deacetylation increases (Tomihata and Ikada, 1997).

\subsection{Chitin and chitosan oligomers}

Although chitin and chitosan are known to have very interesting functional properties in many areas, there is doubt about their level of absorption in the human intestine; their high molecular weights and highly viscous nature may restrict their use in in-vivo systems 


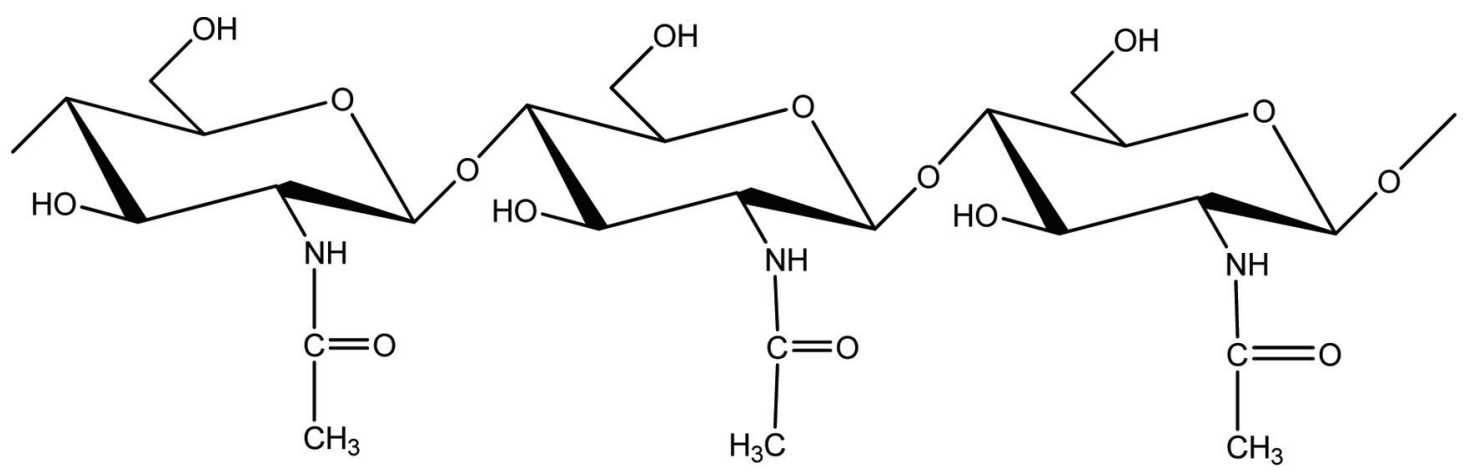

Chitin

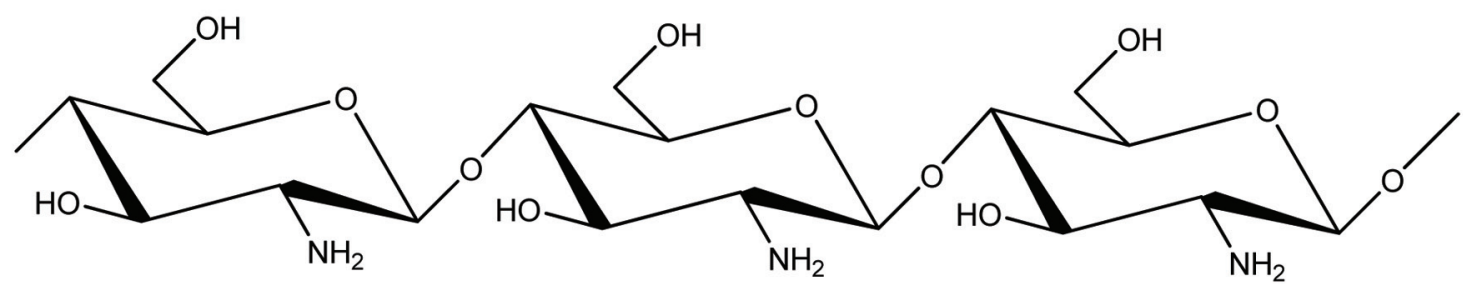

Chitosan

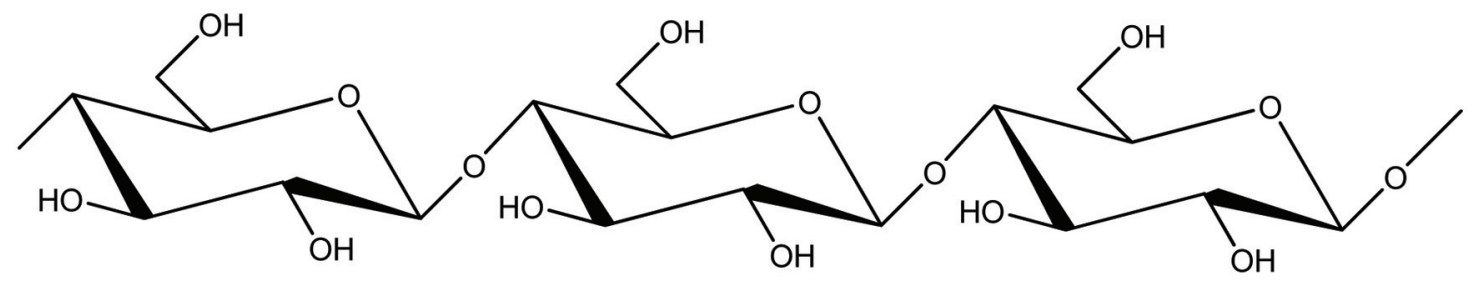

Cellulose

Figure 9. Structure of chitin, chitosan and cellulose.

(Jeon et al., 2000). Therefore, increasing attention has recently been paid to prepare its oligosaccharides. Chitin and chitosan oligomers are composed of $\beta$ - $(1 \rightarrow 4)$ linked $N$-acetylglucosamine and glucosamine units, respectively, with approximately ten residues or less (Jeon et al., 2000). The low-molecular-weight chitin and chitosan oligomers/oligosaccharides (COS) and hetero-chitosan oligosaccharides exhibit antitumor, immuno-enhancing, antibacterial, antifungal, free radical scavenging, ACE inhibitory and anticoagulant activities (Jeon and Kim, 2002; Jeon et al., 2001; Park et al., 2004; Tsukada et al., 1990). Oligomers from chitin and chitosan are prepared by two hydrolytic methods, chemical hydrolysis (acidic hydrolysis) and enzymatic hydrolysis (Jeon, et al., 2000). Acidic depolymerization is carried out by $\mathrm{HCl}$, hydrofluoric acid, nitrous acids, acetic acid, or sulfuric acid. However, most of the acidic hydrolyses procedures have reported production of oligomers with a low degree of polymerization (DP), mainly from monomer to tetramer with quantitative yields (Sakai et al., 1990; Kurita et al., 1993; Takahashi et al., 1995; Horowitz et al., 1957). However, removing these strong acids leads to environmental pollution and the yields of the oligomers with relatively higher DP, such as pentamer to heptamer, were low because physiologi- cal effects have been expressed mainly by high DP oligomers. In contrast to chemical hydrolysis, enzymatic hydrolysis of chitin and chitosan by chitinase, chitosanase, lysozyme, and cellulase has several benefits to produce more readily available oligomers with a high DP with milder reaction conditions (Aiba, 1994; Usui et al., 1987, 1990). Besides, some other non-specific enzymes such as lipases, papain, hemicellulases, pectinases, pepsin, and pronase were also used to produce chitosan oligosaccharides (COS) (Abdel-Aziz et al., 2014). These enzymes all catalyze the cleavage of $\beta$-1,4-glycosidic linkages in chitosan in a random manner to form oligomers. Compared with the specific enzymes (chitinase, chitosanase), the non-specific enzymes including cellulase and hemicellulase are cheaper, commercially available, and with the same efficiency (Hamed et al., 2016). Uchida et al. (1989) explained that the enzymatic hydrolysis was a useful method for preparation of oligomers from chitin and chitosan because the yield of high DP oligomers was greater in enzymatic hydrolysis than in acid hydrolysis. Furthermore, depending on the type of enzyme employed, chitosan oligomers with specific chain lengths may be produced for certain applications (Jeon et al., 2000). Due to their solubility in water, chitosan oligomers serve best in rendering their benefits 


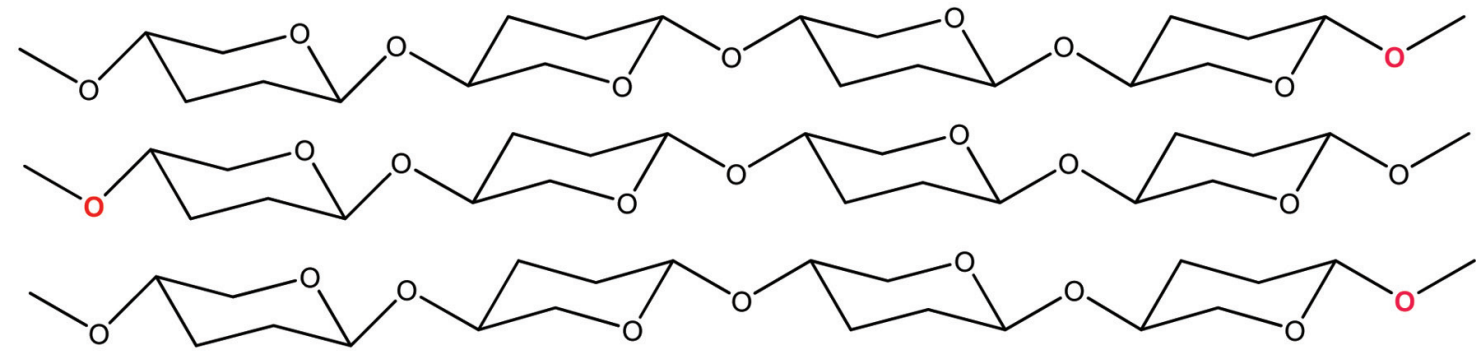

a

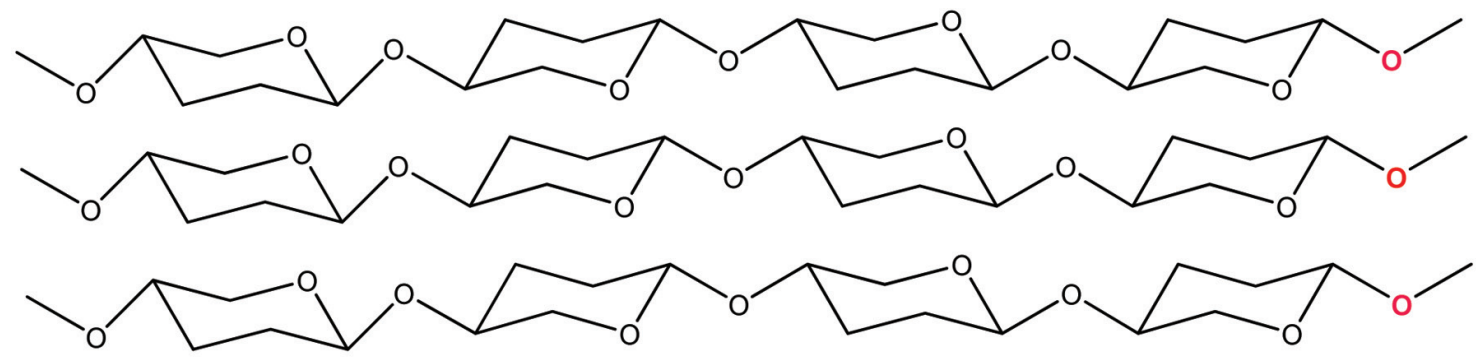

b

Figure 10. $\alpha$ (a) and 6 (b) polymorphic forms of Chitin. Source: Adapted from Mourya and Inamdar (2008).

under normal physiological conditions and in foods with neutral pH (Shahidi, 2007).

\subsection{Glucosamine and N-acetyl glucosamine}

Glucosamine (GlcN) and its acetylated form, $N$-acetyl glucosamine (GlcNAc), are the monomers of chitosan and chitin, respectively. GlcN is the dominating monomer in chitosan, while chitin contains more GlcNAc than GlcN (Zamani et al., 2008). Glucosamine represents one of the most commonly used drugs to treat osteoarthritis (Shikhman et al., 2001). GlcN and, to a higher degree, GlcNAc, possess a unique range of anti-inflammatory activities (Shikhman et al., 2001). Meanwhile, glucosamine in the human body participates in the structure of cartilage and works to stimulate joint function and repair. It has proven to be effective in numerous scientific trials for easing osteoarthritis pain, aiding in the rehabilitation of cartilage, renewing synovial fluid, and repairing joints that have been damaged from osteoarthritis (Mankin et al., 1986; Hauselman, 2001). Hochberg et al. (2016) conducted double-blind Multicentre Osteoarthritis intervention trial with 606 patients with Kellgren and Lawrence grades 2-3 knee osteoarthritis and moderate-to-severe pain from France, Germany, Poland, and Spain. The patients were randomized to receive $400 \mathrm{mg}$ chondroitin sulfate (CS) plus $500 \mathrm{mg}$ glucosamine hydrochloride $(\mathrm{GH})$ three times a day or $200 \mathrm{mg}$ celecoxib every day for 6 months. It was finally found that $\mathrm{CS}+\mathrm{GH}$ had comparable efficacy to celecoxib in reducing pain, stiffness, functional limitation and joint swelling/effusion after 6 months in patients with painful knee osteoarthritis, with a good safety profile. More recently, serum GlcNAc concentrations were used as a biomarker in diagnosing multiple sclerosis (MS) subtype in a patent; this could also be used in patients with various levels of neurodegenerative severity, including clinical severity of MS in MS subjects, as an indicator of MS progression (Demetriou et al., 2018). In another patent, glucosamine was also added as functional ingredient in the sweetener (Prakash and Dubois, 2015).

Glucosamine products for arthritis are usually formulated as the hydrochloride salt or glucosamine sulfate (Figure 12) and are often combined with chondroitin sulfate (Abdel-Fattah and Hammad, 2001). The preparation of glucosamine hydrochloride from chitin is a simple hydrolysis reaction. During this reaction, chitin is deacetylated and depolymerized to glucosamine hydrochloride in the presence of hydrochloric acid solution (Shahidi, 2007). However, it was later reported that glucosamine hydrochloride was ineffective in the treatment of osteoarthritis mainly because of its pharmacokinetics limitations (Reginster et al., 2018). Glucosamine sulfate which is made from glucosamine hydrochloride by adding either potassium or sodium sulfate and co-crystallizing the resulting mixture was approved as a prescription drug in Europe and elsewhere (Mojarrad et al., 2007).

\subsection{Application of chitin, chitosan and their oligomers and monomers}

\subsubsection{Application in the Food Industry}

Edible film

Current trends in consumer preferences toward mildly preserved, fresh, and healthy foodstuffs have triggered innovation in food, 


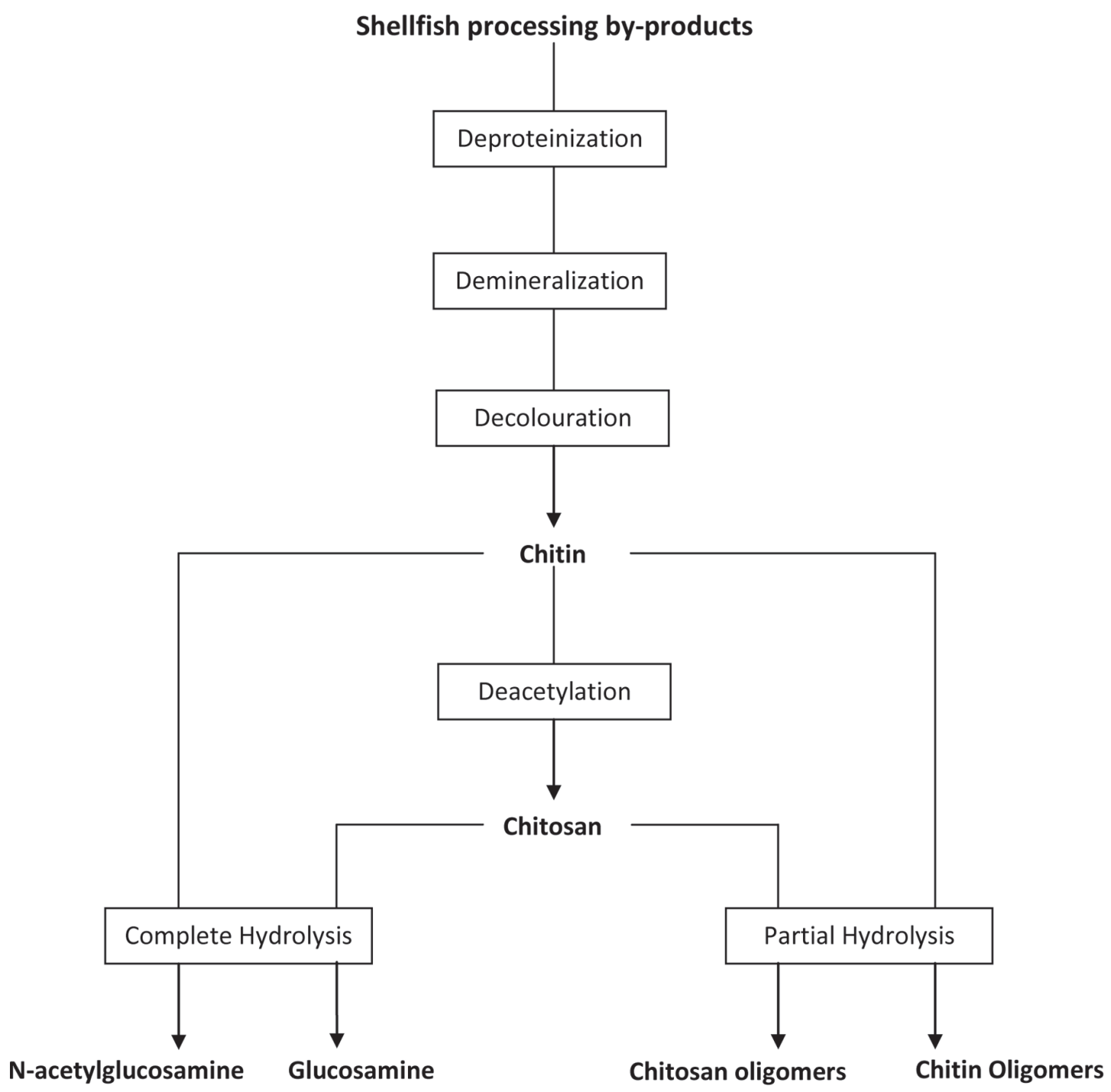

Figure 11. Schematic representation of industrial manufacture of chitin, chitosan and their oligomers and monomers from shellfish processing byproducts. Source: Adapted from Shahidi (2007).

food coating, and food packaging applications. As a result, in recent years much attention has been paid to the development of biocide edible films with antimicrobial properties, which improve food safety and extend shelf life. Antimicrobial packaging is one of the most promising active packaging systems (Fernandez-Saiz et al., 2009). Chitin/chitosan represents interesting properties such

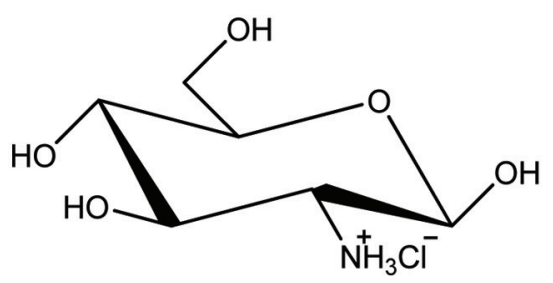

a

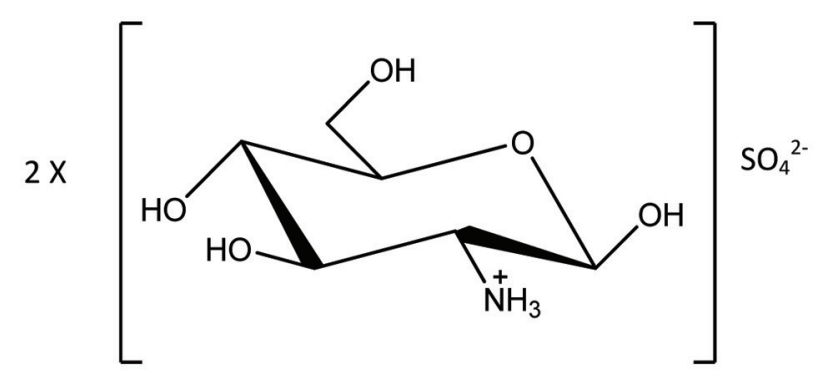

b

Figure 12. Chemical structures of (a) D-glucosamine hydrochloride, and (b) D-glucosamine sulphate. Source: Adapted from Abdel-Fattah and Hammad (2001). 
as excellent film-forming capacity and gas and aroma barrier properties under dry conditions, which makes it a suitable material for designing food coatings and packaging (Cancer, 2005; Shankar et al., 2015; Qin et al., 2016). In addition to this, chitosan is readily soluble in various acidic solvents and has high antimicrobial activity against many pathogenic and spoilage microorganism, including fungi, gram-positive and gram-negative bacteria (Coma, 2008). Due to its ability to form semi-permeable film, chitosan coating can be expected to modify the internal atmosphere as well as decreasing the transpiration loss and delay the ripening of fruits (Shahidi et al., 1999). Moreover, the FDA has approved the use of chitosan as an edible film to protect foods.

Films from aqueous chitosan are clear, tough, flexible, and provide good oxygen barrier properties (Sanford, 1989; Kaplan et al., 1993). Carbon dioxide permeability could be improved by methylation of polymers (Bourtoom, 2008). Butler et al. (1996) observed that films from chitosan were rather stable and their mechanical and barrier properties changing only slightly during storage. Chitosan coatings could be used on fruit and vegetable products such as strawberries, cucumbers, bell peppers as antimicrobial coating (El Ghaouth et al., 1991a, 1991b), and on apples, pears, peaches and plums as gas barrier (Elson and Hayes, 1985; Davies et al., 1989). Abugoch et al. (2016) used chitosan as an ingredient of edible film coating for fresh blueberries which was able to control the growth of molds and yeasts during 32 days of storage. Meanwhile, the tomatoes coated with quinoa protein/ chitosan edible film showed a significant decrease in $B$. cinerea growth after 7 days at $5{ }^{\circ} \mathrm{C}$ (Robledo et al., 2018). Besides chitosan, increasing the concentration of chitin nanoparticles from 0 to $5 \%$ also significantly enhanced the anti-fungal properties of the gelatin-based nanocomposite films against Aspergillus niger (Sahraee et al., 2017). Meanwhile the nanocomposite films with added chitin nano-whiskers/chitin nanofibrils exhibited strong antimicrobial activity against gram-positive Listeria monocytogenes and but not against gram-negative Escherichia coli (Qin et al., 2016; Shankar et al., 2015).

\section{Color Stabilization Agent}

Chitin and chitosan have the potential to act as color stabilizers in foods due to their unique molecular structures (Knorr, 1991). Chitosan has an extremely high affinity for many classes of dyes, including disperse, direct, reactive, acid, vat, sulfur, and naphthol dyes (Agullo et al., 2003). Georgantelis et al. (2007) reported that inclusion of chitosan combined with rosemary extract in fresh ground beef patties provided a synergistic effect in preventing lipid oxidation and stabilizing the deep red color of the product. The rate of diffusion of dyes on chitosan was similar to those on cellulose, which is already being used by the food processing industry to improve food functionality and quality in Korea and Japan for the past three decades (Kumar, 2000).

\section{Antimicrobial Agent}

Chitin, chitosan and their oligomers, have a broad antimicrobial spectrum for gram-negative, gram-positive bacteria, mold, and yeasts (Roller and Covill, 1999; Shahidi, 2007; Sudarshan et al., 1992; Wang, 1992; Yalpani, et al., 1992). However, their exact mechanism of antibacterial action remains illusive. Many of the physical and chemical properties of these compounds are related to the environmental conditions such as $\mathrm{pH}$, type of microorganism, and neighboring components; and its structural characteristics such as molecular weight (MW), degree of deacetylation, derivative form, its concentration, and original source (Hosseinnejad and Jafari, 2016). Herein, pH, MW, and degree of deacetylation together provide an excellent estimation of the charge density (Avila Rodríguez et al., 2018). Possible and accepted antimicrobial mechanisms for chitosan have been reported in the existing literature (Agullo et al., 2003; Goy et al., 2009).

The most acceptable mechanism of action is reported to be the interaction between positively charged chitin/chitosan molecules and negatively charged microbial cell membranes (Shahidi 2007; Goy et al., 2009). In this model, the interaction is mediated by the electrostatic forces between the protonated amino groups, the $\mathrm{NH}_{3}^{+}$ groups, and the negatively charged residues (Tsai and $\mathrm{Su}, 1999$ ), presumably by competing with $\mathrm{Ca}^{2+}$ for electronegative sites on the membrane surface (Young and Kauss, 1983). This electrostatic interaction promotes changes in the properties of membrane wall permeability, thus provoking internal osmotic imbalance and consequently inhibits the growth of microorganisms (Hadwiger et al., 1981; Shahidi et al., 1999). In addition to this the hydrolysis of the peptidoglycans in the microorganism wall, leading to the leakage of intracellular electrolytes such as potassium ions and other low molecular weight proteinaceous constituents (e.g. proteins, nucleic acids, glucose, and lactate dehydrogenase) (Chen et al., 1998; Papineau et al., 1991; Sudarshan et al., 1992; Devlieghere et al., 2004; Fang et al., 1994). Therefore, the greater the number of cationized amines, the higher will be the antimicrobial activity (Másson et al., 2008; Yalpani et al., 1992). This suggests that chitosan has a higher activity than that found for chitin and this has been confirmed experimentally (Tsai and $\mathrm{Su}, 1999$ ).

Effectiveness of chitosan on gram-positive or gram-negative bacteria is somewhat controversial. Some authors have stated that chitosan generally shows stronger effects for gram-positive than for gram-negative bacteria (Coma et al., 2003; Dutta et al., 2009; Jeon et al., 2001; No et al., 2002). Conversely, it has been demonstrated that hydrophilicity in gram-negative bacteria is significantly higher than that in gram-positive bacteria, making them more sensitive to chitosan (Chung et al., 2004). More recently, it was reported that antibacterial activity was further enhanced for gramnegative bacteria with decreasing MW, whereas the opposite effect was observed with gram-positive bacteria (Younes et al., 2014). The charge density on the cell surface is a determinant factor that establishes the amount of adsorbed chitosan. More adsorbed chitosan would evidently result in greater changes in the structure and in the permeability of the cell membrane (Goy et al., 2009).

Another possible mechanism is the binding of chitosan with microbial DNA, which leads to the inhibition of the mRNA and protein synthesis via the penetration of chitosan into the nuclei of the microorganisms (Sudarshan et al., 1992; Sebti et al., 2005; Hadwiger, et al., 1981). According to Raafat et al. (2008), chitosan acts essentially as an outer membrane disruptor rather than a penetrating material. A third mechanism that might be involved is by its action as a chelating agent. In this, chitosan has the ability to bind with essential nutrients required for microbial growth, hence making them unavailable (Cuero et al., 1991; Roller and Covill, 1999).

The correlation between the degree of acetylation and the antimicrobial activity of chitosan has been studied (Kendra and Hadwiger, 1984). Tsai et al. (2002) examined the effects of the degree of deacetylation and preparation methods on the antimicrobial properties of chitin and chitosans. It was found that chemically(CH-chitin) and microbiologically-prepared (MO-chitin) chitin could undergo further chemical deacetylation to produce chitosan with different degrees of deacetylation. The investigation of Tsai et al. (2002) showed that microbiologically-prepared chitin that was 
deacetylated by various proteases had no antimicrobial activity. However, for chitosan, the degree of deacetylation determines the content of free amino groups and hences the higher the degree of deacetylation, the higher is the solubility in acidic conditions; this makes the polymer having a higher positive charge, which would be expected to have stronger antibacterial activity, even to a greater extent than that on fungi (Shahidi 2007; Tolaimate et al., 2003). In the study of Byun et al. (2013), the chitosan prepared from ground shells of crab legs (degree of deacetylation $81.56 \%$ ) showed a superior antibacterial activity than that of chitosan from the entire shell (degree of deacetylation 62.71\%). However, Park et al. (2004) found that $25 \%$ acetylated chitosan had a higher inhibitory activity than that of $10 \%$ acetylated chitosans. Later on, studies of Hongpattarakere and Riyaphan (2008) and Takahashi et al. (2008) demonstrated that chitosan with a higher degree of deacetylation had a better inhibitory activity on both E.coli, S.aureus and Candida albicans. Martorana et al. (2002) have compared the antimicrobial effect of chitosan and water-soluble $\mathrm{N}$-acetylated chitosan on Salmonella typhimurium. The antimicrobial activity of acetic acid used as a diluent of the polymer was also investigated. Their results demonstrated that chitosan improves the performance of acetic acid as an antimicrobial agent. Chitosan and water-soluble $\mathrm{N}$-acetylated chitosan had similar antimicrobial activity on Salmonella typhimurium growth at $\mathrm{pH} 5.5$, although the degree of deacetylation of both products was very different.

Generally, chitosan has been reported as being very effective in inhibiting spore germination, germ tube elongation, and radial growth (El ghaouth et al., 1992; Sashai and Manocha, 1993). The chelating properties of chitosan makes it a potential antifungal agent (Hirano and Nagao, 1989). Mild hydrolysis of chitosan resulted in improved microbial inactivation in saline medium and greater inhibition of several spoilage yeast growth in laboratory media, but highly degraded products of chitosan exhibited no antimicrobial activity (Agullo et al., 2003). In pasteurized apple-elderflower juice stored at $7{ }^{\circ} \mathrm{C}$, addition of $0.3 \mathrm{~g}$ of chitosan per liter eliminated yeast entirely for the duration of the experiment while the total counts and the lactic acid bacterial increased at a slower rate than that in the control treatment (Agullo et al., 2003). Wang (1992) observed that a much higher concentration of chitosan (1$1.5 \%$ ) was required for a complete inactivation of Staphylococcus aureus after two days of incubation at pH 5.5 or 6.5 in the medium. Bacillus cereus required a chitosan concentration of $0.02 \%$ for a bactericidal effect, while Escherichia coli and Proteus vulgaris showed minimal growth at $0.005 \%$, and complete inhibition at $>0.0075 \%$ (Simpson et al., 1997). Wang (1992) observed complete E. coli inactivation after a two-day incubation period with concentrations of 0.5 or $1 \%$, at $\mathrm{pH} 5.5$. Meanwhile, other authors have reported different results. These variations were suggested to be due to the existing differences in the degree of acetylation of chitosan; a greater degree of acetylation was less effective (Darmadji and Izumimoto, 1994).

The antibacterial effect of three kinds of chitooligosaccharides such as relatively high molecular weights (HMWCOS-10 kDa), medium molecular weight (MMWCOS-5 kDa), and low molecular weight (LMWCOS-1 $\mathrm{kDa}$ ) have been evaluated by Jeon et al. (2000) against various microorganisms. They reported that the growth of most bacteria tested was inhibited by chitooligosaccharide treatments, in particular by HMWCOS, although chitosan treatment showed a better inhibitory effect. Furthermore, they observed that the molecular weight of chitooligosaccharides was critical for microorganism inhibition and required to be higher than $10 \mathrm{kDa}$. Similarly, Li et al. (2010) evaluated the inhibitory activity of chitosan solution $(3,50,1,000 \mathrm{kDa})$ against E. coli and found all of them inhibited the growth of E. coli, but chitosan of $50 \mathrm{kDa}$ was most effective. The main reason is that HMW chitosan cannot pass through the microbial membrane and hence stacks on the cell surface, which blocks nutrient transport into the microbial cell membrane, resulting in cell lysis. Generally, the chitooligosaccharides have more effective activity against pathogens than non-pathogens, except in the case of lactic acid bacteria (Jeon et al., 2000).

\subsubsection{Application in functional foods/nutraceutical}

Chitosan is well positioned as a functional food ingredient to lower cholesterol and to decrease fat absorption, and therefore decrease obesity (Preuss and Kaats, 2006; Shahidi 2007; Hayes et al., 2008b). Several studies have established that the ability of appropriately prepared chitosan decreases both cholesterol and fat absorption levels in both animals and humans when delivered at low dosages in supplements or in food (Yuji et al., 1993; Bokura and Kobayashi, 2003; Schiller et al., 2001; Kanauchi et al., 1995). Some studies have shown that chitosan can decrease blood cholesterol levels more than $50 \%$ and demonstrating it as an effective hypocholesterolemic agent (Jameela et al., 1994). Additionally, a preliminary human trial in which 3-6 g chitosan was consumed every day for 2 weeks resulted in a decrease in LDL-cholesterol by $6 \%$ and concurrently, a boost in high-density lipoprotein (HDL) cholesterol by 10\% (Maezake et al., 1993). Although the exact mechanism of action of chitosan and chitosan oligosaccharides (COS) in LDL cholesterol reduction is not clear, it has been claimed that these molecules act as fat scavengers in digestive tract and remove cholesterol via ionic binding of COS or chitosan with bile salts and acids, thus preventing the formation of lipid micelles during digestion, which results in excretion of fat and cholesterol (Muzzarelli et al., 2000; Ylitalo et al., 2002; Remunan-Lopez, et al., 1998). Another hypothesis suggests that chitosan and its oligomers can directly trap lipids and fatty acids (Tanaka et al., 1997). It has also been suggested that the lowering of cholesterol levels is a result of the ability of COS to increase neutral sterol excretion (Kim and Rajapakse, 2005). Despite animal studies demonstrating the involvement of COS in fat excretion, some studies have shown that certain low molecular weight COS, usually $<2,000 \mathrm{Da}$, fail to absorb dietary fats (Ikeda et al., 1993). Sugano et al. (1998) have investigated the relationship between intakes of different molecular weight COS and hypocholesterolemic effect in mice and have reported that almost all chitosan preparations could prevent increase in blood cholesterol levels at a $5 \%$ dietary level. In addition, earlier work of Sugano et al, (1992) showed that highly depolymerized chitosans are not effective in lowering cholesterol levels. Azuma et al. (2014) evaluated the anti-obesity effects of chitin nanofibers (CNFs) and surfacedeacetylated chitin nanofibrils (sda-CNFs) in a mouse model of high-fat-diet-induced obesity. The results showed that sda-CNFs suppressed the body weight gain produced by the high-fat diet as well as the serum levels of leptin and TNF- $\alpha$; however, CNFs did not suppress such weight gain.

Apart from anti-obesity, various monomers and oligomers of chitin and chitosan also possess superior anti-inflammatory effects. Earlier reports indicated that GlcN and GlcNAc produce different effects in vitro. For example, GlcN, but not GlcNAc, suppressed interleukin (IL)-8, prostaglandin E2, and nitric oxide synthesis in IL-1 $\beta$-treated synoviocytes (Hua et al., 2007). A recent report showed similar suppressive effects on the levels of serum tumor necrosis factor- $\alpha$ (TNF- $\alpha$ ), IL- 6 concentrations, serum IL-10, transforming growth factor- $\beta$, and IL-2 thorough oral administration of GlcNAc and GlcN in an experimental of rheumatoid arthritis model (Azuma et al., 2012). Moreover, oral administration of 
COS inhibited colon shortening and tissue injury by suppressing myeloperoxidase activation in inflammatory cells, reducing serum levels of pro-inflammatory cytokines tumor necrosis factor- $\alpha$ and IL-6, and prolonged survival time, as well as inhibiting the activation of nuclear factor-kappa B, cyclooxygenase-2, and inducible nitric oxide synthase, in mice IBD (inflammatory bowel disease) model (Azuma et al., 2015c).

Due to the inconsistent clinical data, difficulties in producing a structurally consistent product, the requirement of relatively high dosages for efficacy, and competition from fiber products with FDA health claims such as oats, bran, psyllium fiber, soy protein and phytosterol esters, chitosan is not widely used as a functional ingredient in North America (Hayes et al., 2008a). However, chitosan produced by Primex of Norway has GRAS approval and in Japan several foods including soybean paste, potato chips, and noodles are available with added chitosan, as cholesterol-lowering functional foods (Alasalvar et al., 2002).

\subsubsection{Enzyme immobilization on chitin- and chitosan-based materials}

The properties of immobilized enzymes are governed by the properties of both the enzyme and the support material (Kennedy and Cabral, 1983; Tischer and Wedekind, 1999). However, it is recognized that there is no universal support for all enzymes and their applications. The main goal of immobilization is the re-use of enzymes for an extended period (Shahidi et al., 1999). As functional materials, chitin and chitosan offer a unique set of characteristics, which are indispensable for for immobilizing enzymes. Mainly, these include high affinity to proteins, availability of reactive functional groups for direct reactions/interactions with enzymes and for chemical modifications, hydrophilicity, mechanical stability and rigidity, regenerability and ease of preparation in different geometrical configurations that provide the system with permeability and surface area suitable for a chosen biotransformation (Krajewska, 2004). Understandably, nontoxic and biocompatible materials are also required for food, pharmaceutical, medical and agricultural applications. Furthermore, to respond to the growing public health and environmental awareness, the materials should be biodegradable, and to be economical and inexpensive. Owing to these characteristics, chitin- and chitosan-based materials are widely exploited as enzyme immobilization support.

Chitin and chitosan-based materials, which are obtained at a relatively low cost from shells of shellfish (mainly crabs, shrimps, lobsters and krills) processing discards could be used in the form of powders, flakes and gels of different geometrical configurations as enzyme immobilization support (Krajewska, 2004; Shahidi et al., 1999). The immobilization of enzymes such as $\alpha$-amylase, $\beta$ amylase, glucose isomerase, and amyloglucosidase on krill chitin activated by formaldehyde was shown to be initiated by the generation of the hydrated form of formaldehyde that condenses with free amino groups of chitin, forming Schiff's bases and dihydroxymethyl derivatives of aldehyde (Synowiecki and Al-Khateeb, 2003). These Schiff's bases might be responsible for immobilization of enzymes by reacting with various functional groups of the enzymes, thus forming methylene bridges (Shahidi et al., 1999). A similar study by Han and Shahidi (1995) reported 20-29\% activity retention of crude seal gastric proteases after immobilization on glutaraldehyde-treated chitin. The characteristics of the immobilized crude native seal gastric proteases were similar to those of chymosin. Several other enzymes such as lipase, lysozyme, Lasparaginase, nitrite hydratase or amylase among others have been immobilized in these polymers (Cappannella et al., 2016; Gilani et al., 2016; Kudryashova et al., 2015; Pawar and Yadav, 2014). The immobilization of penicillin $\mathrm{G}$ acylase on different physical forms of chitosan, namely beads, particles and powder by Braun et al. (1989) demonstrated that magnetic beads are suitable supports for enzymes as they can be easily removed from the reaction medium. Another study by Siso et al. (1997) demonstrated that microencapsulation in chitosan beads was an effective enzyme immobilization method for invertase and $\alpha$-amylase. Use of immobilized enzymes in cheese production provides a promising approach for the rational use of rennin and the inherent advantages of continuous reactor systems (Agullo et al., 2003). Doğaç and Teke (2016) recently reported the effective removal of urea thorough natural polymer-conjugated magnetic featured urease systems. Bilal et al. (2017) immobilized horseradish peroxidase in chitosan beads by an entrapment method and used it for efficient degradation of textile dyes. In addition, enzyme stabilization against microbial degradation in real bioremediation processes was developed by encapsulation of laccase in chitosan nanoparticles (Bilal et al., 2017). Furthermore, Zhao et al. (2016) immobilized glucose isomerase using silica/chitosan hybrid microspheres via an easy approach through in situ encapsulation of glucose isomerase. The results demonstrated that the immobilized glucose isomerase had much wider stability range of $\mathrm{pH}$, temperature, ions, storage, and operation in comparison with the free glucose isomerase.

\subsubsection{Application in bioremediation technology}

Chitin and its derivatives from shellfish wastes could be utilized in bioremediation process as an electron donor (Hayes et al., 2008b). Bioremediation can be defined as any process that uses microorganisms or their enzymes to return the environment altered by contaminants to its original condition. In order for microbes to clean up harmful chemicals, the right temperature, nutrients, and amount of oxygen must be present (Hayes et al., 2008b). These conditions allow the microbes to grow and multiply-and eat more chemicals. When conditions are not right, microbes grow too slowly or may die or create more harmful chemicals. Furthermore, the microorganism injection process is both expensive and labor intensive and the injection process can often result in excessive microbial growth (Brennan et al., 2006b; Hayes et al., 2008b). The dry-cleaning chemical, perchloroethylene (PERC), and its daughter products trichloroethene (TCE), cis-1,2-dichloroethene (DCE), and vinyl chloride (VC) are common groundwater contaminants in industrialized nations (Neumann et al., 1994). The use of chitin from crab shell waste and mixture of chitin and corncobs as an electron donor source was investigated by Brennan et al. (2006a) to stimulate reductive dechlorination of the dry-cleaning contaminant by in situ bioremediation technology. In situ bioremediation of chlorinated ethenes using chitin from crab shell waste as an electron donor source and the anaerobic culture Desulfuromonas michiganensis strain BB1 demonstrated several advantages over the use of conventional liquid electron donor systems. Thus, a diverse number of chitin derivatives provided a wide range of electron donor and nutrients which could be used in general treatment of contaminants other than chlorinated ethenes and the buffering effect provided by residual calcium carbonate contained in the shell material had a positive effect on dechlorination (Brennan et al., 2006a and 2006b).

Other significant development in the new range of applications of chitosan is the removal of metal ions from wastewater. The contamination of heavy metals such as $\mathrm{As}, \mathrm{Cu}, \mathrm{Cd}, \mathrm{Pb}, \mathrm{Cr}$, $\mathrm{Ni}, \mathrm{Hg}$ and $\mathrm{Zn}$ in the environment is of great concern due to its potential impact on human and animal health. Non-biodegradable 

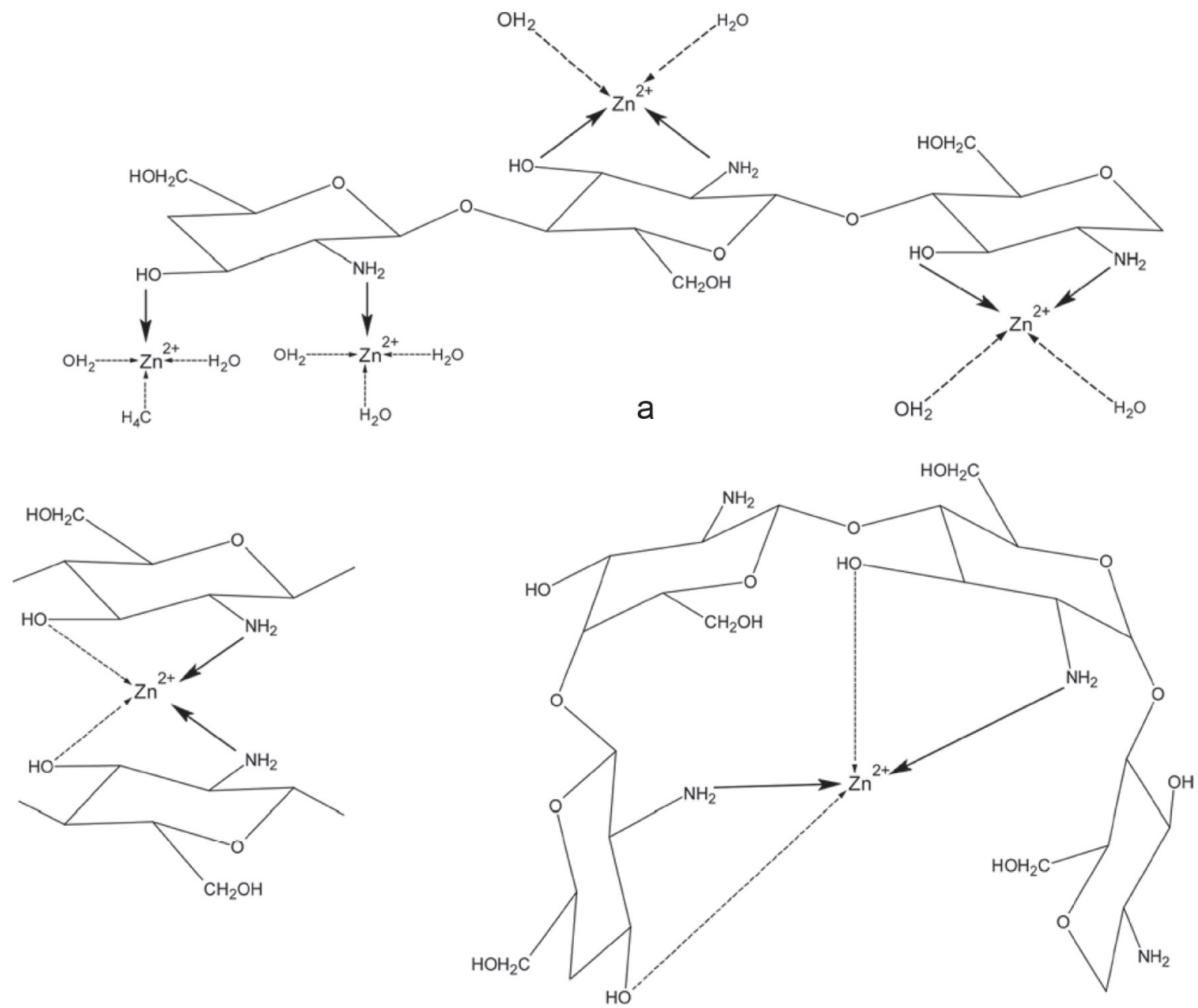

b

C

Figure 13. The possible structure of Chitosan-Zn complexes. Source: Adapted from Gerente et al. (2007).

heavy metals can accumulate in living tissues and subsequently become concentrated in the food chain with potentially detrimental effects on a variety of living species. They cannot be destroyed biologically but are only transformed from one oxidation state or organic complex to another (Garbisu and Alkorta, 2001; Gisbert et al., 2003). Therefore, heavy metal pollution poses a great potential threat to the environment and human health. Cheaper and effective technologies are needed to protect the natural environment and biological lives. Chitosan adsorbs significant amounts of metal ions and is valued for being readily available, cost effective, environmentally friendly, and biodegradability characteristics. Thus, it has generated considerable interest in assessing its feasibility to remove metal ions over a wide range of effluent systems and types (Gerente et al., 2007; Singh and Nagendran, 2016; Irawan et al., 2018). Chitin and chitosan derivatives have different functional groups $\left(-\mathrm{NH}_{2},-\mathrm{OH}\right)$ which interact with heavy metal ions, dissolved organic solids, colored dyes, and other contaminants. They particularly help remove targetted metal ions from aqueous solutions when the $\mathrm{pH}$ is close to neutral (Sarode et al., 2019). The flexibility of the polymer chain is also one of the reasons why chitin-/ chitosan-based biopolymer is an excellent biosorbent (Benavente et al., 2011). Furthermore, chitosan shows a cationic behavior in acidic media; the protonation of amine groups leads to adsorption by ion exchange (Guibal, 2004). Besides, the order of efficacy of chitosan for the removal of metal ions was $\mathrm{Cu}>\mathrm{Ni}>\mathrm{Co}>\mathrm{Mn}$ (Ishii et al., 1995). The metal sorption, however, might have different mechanisms including ion exchange, ion-pairing, chelation, and electrostatic attraction depending on the $\mathrm{pH}$ and solution composition, since such parameters influence the protonation of biopolymers like chitosan and the speciation of metal ions (Sarode et al., 2019). Figure 13 shows the possible structures of chitosan-metal complexes. It has been demonstrated that crab shell waste adsorbs heavy metals more effectively compared to other biological materials (Hayes et al., 2008b; Barriada et al., 2007). Furthermore, chitosan has been examined to treat wastewater from the milk processing plant, and the textile industry (Hayes et al., 2008b).

\subsubsection{Application in biomedical and pharmaceutical Industries}

Chitin and chitosan are the main polysaccharides currently used in the biomedical and pharmaceutical industries due to their interesting biopharmaceutical characteristics (Shahidi 2007; Prabaharan, 2008; Rinaudo, 2007). The main current development is in medical 
and pharmaceutical applications as orthopedic/periodontal material, wound-dressing material, tissue engineering or controlled drug/ gene delivery (Agboh and qin, 1996; Deepthi et al., 2016; Felse and Panda, 1999; Hirano and Midorikawa, 1998; Hu et al., 2013; Huang et al., 2012; Kumar, 2000; Liu et al., 2016; Manivasagan et al., 2016; Ohkawa et al., 1999; Senda et al., 2001).

\section{Wound healing and wound dressing}

Both chitin and chitosan possess biological properties such as biocompatibility, biodegradability, hemostatic activity, healing acceleration, non-toxicity, non-allergenicity, adsorption properties and anti-infection, among other advantages for wound healing (Shahidi 2007; Shelma et al., 2008; Rinaudo, 2007). Chitin and chitosan have both stimulatory and inhibitory effects on proliferation of human dermal fibroblasts and keratinocytes (Howling et al., 2001). The polymeric unit $N$-acetyl-D-glucosamine initiates fibroblast proliferation and helps in ordered collagen deposition and stimulates increased level of natural hyaluronic acid synthesis at the wound site that helps in faster wound healing and scar prevention (Shelma et al., 2008). Chatelet et al. (2001) showed that the degree of deacetylation of chitosan plays a key role in cell adhesion and proliferation. They observed that the higher the degree of deacetylation of chitosan, the lower its cell adhesion on films; fibroblasts appear to adhere twice as much as keratinocytes on these materials. Further, they found that keratinocyte proliferation increases when the degree of deacetylation of chitosan films decreases. Both chitin and chitosan have a great influence on different stages of wound healing in experimental animal models (Howling et al., 2001). The novel non-woven microfiber mats fabricated with chitin/chitosan-glucan complex of Schizophyllum commune exhibited not only excellent surgical wound healing ability in rat models but also antibacterial activity against various bacteria including Escherichia coli (E. coli; $-\mathrm{G})$; Klebsiella pneumoniae (K. pneumoniae; $-\mathrm{G}$ ), Basillus subtilis (B. Subtilis; $+\mathrm{G}$ ) and Staphylococcus aureus (S. aureus; $+\mathrm{G}$ ) (Abdel-Mohsen et al., 2016). In addition to wound healing, chitosan has been demonstrated as an excellent wound dressing film which provides optimum microenvironment for healing by removing excess wound exudates and allowing continuous tissue reconstruction (Shelma et al., 2008). It has been demonstrated that the use of chitosancontained bandages called 'shrimp' bandage can stop capillary bleeding and stanch severe arterial hemorrhaging (Taylor, 2009). In order to create a moist environment for rapid wound healing, a hydrogel sheet composed of a blended powder of alginate, chitin, chitosan, and fucoidan $(60: 20: 2: 4, \mathrm{w} / \mathrm{w} / \mathrm{w} / \mathrm{w})$ has been developed as a functional wound dressing which was expected to effectively interact with and protect the wound in rats, providing a good moist healing environment with exudates (Azuma et al., 2015a).

\section{Controlled drug delivery systems}

Drug release is also an important application for chitin and chitosan, which exhibit the required physiological characteristics such as biocompatibility blood biocompatibility, bioadhesivity, biodegradability, and low toxicity, among others (Rinaudo 2007; Hirano and Noishiki, 1985). Polymers used as drug carriers are required to be biodegradable to prevent polymer accumulation in the body. However, since the rate of biodegradation affects drug action, it must be carefully controlled. Chitin and chitosans have recently been examined in drug delivery systems in pharmaceutical industry due to their ability to promote long-term release of in- corporated drugs compared to other polysaccharides (Masotti and Ortaggi, 2009; Je and Kim 2006; Hon, 1996). Chitin can be used alone or mixed with other polymers (Mi et al., 2002). It has been demonstrated that carboxymethylchitin microspheres were preferentially located and retained for long in liver and spleen after i.v. injection. It indicates that these microspheres can be used to target drug delivery to the liver (Hata et al., 2000).

Chitosan has also been tested for the development of conventional and novel drug delivery systems (Rinaudo, 2007; Prabaharan, 2008). It has the special feature of adhering to mucosal surfaces, a fact that makes it a useful polymer for mucosal drug delivery. However, practical use of chitosan has mainly been confined to the unmodified forms due to its low solubility at the physiological $\mathrm{pH}$ of 7.4, limiting its use as absorption enhancer in, for example, nasal or peroral delivery systems. Another limitation arises from its rapidly adsorbing water and a higher swelling degree in aqueous environments leading to fast drug release (Prabaharan and Mano, 2007). Therefore, chemically modified chitosan has great utility in controlled release and targeting studies of almost all classes of bioactive molecules. Among the possible modifications, graft copolymerization on chitosan introduces desired properties and expands the field of the potential applications of chitosan by choosing various types of side chains (Prabaharan, 2008). Besides, the stearic acid-g-chitosan oligosaccharide micelles (CSO-SA) have been presented as potential candidates as intracellular antitumor agent delivery carrier of podophyllotoxin (PPT) (Huang et al., 2012). The cytotoxicity of CSO-SA/PPT micelles against human breast carcinoma (MCF-7) cells, human lung cancer cells (A549) and human hepatoma cell line (Bel-7402) were higher than that of free PPT formulation due to the faster PPT transport into tumor cells mediated by CSO-SA micelles. Compared with chitin and chitosan, COS has outstanding advantages because of its accessibility for coupling with the primary amino groups and hydroxyl groups of each polymer subunit and the cationic nature that allows ionic crosslinking, thus it is mainly suitable for polymer-drug conjugates. COS has been improved via modification with hydrophobic residues, including cholesterol, deoxycholic acid, tocopherol, and alkyl groups. This hydrophobically improved COS can form self-assembled nanoparticles, which can be used as carriers for gene and tumor-targeted drug delivery (Manivasagan et al., 2016).

\section{Gene therapy as a non-viral DNA carrier}

Gene therapy is now seen as a promising approach for the treatment of a broad spectrum of health problems. Chitin and chitosan have been extensively studied as non-viral DNA carriers for gene delivery and therapy (Masotti and Ortaggi, 2009). Due to their cationic nature, chitin and chitosan can interact electrostatically with the negatively charged plasmids to form self-assembling polyelectrolyte polymers (Lee et al., 2002; Garnett, 1999). Although chitosan carries a strong positive surface charge at acidic $\mathrm{pH}$ due to its primary amino groups which could bind to negatively charged DNA, waterinsolubility and low transfection efficiency are the major disadvantages (Je, Cho and Kim, 2006). Je et al. (2006) modified chitin at C6 position to produce a water-soluble aminoethylchitin (AEC), which was able to complex with DNA and to form AEC/DNA nanoparticles. In addition to good DNA binding ability, modified chitin showed high protection of DNA from nuclease and serum, and also exhibited low cytotoxicity. In vivo antitumor activity study of $\mathrm{Hu}$ et al. (2013) showed that low-molecular-weight polyethylenimineconjugated stearic acid-g-chitosan oligosaccharide (CSOSA-g-PEI)/ plasmid pigment epithelium-derived factor formulation could effectively suppress the tumor growth (above 60\% tumor inhibition) 
without systematic toxicity against animal body after intravenous injection. In this study, the synthesized CSOSA-g-PEI was found to have a good ion-buffer capability and DNA-binding capacity, which could form positively charged nano-sized particles $(100-150 \mathrm{~nm})$ with plasmid DNACSOSA-g-PEI. In addition, it also had a much lower cytotoxicity and corresponding transfection efficiency in comparison with Lipofectamine 2,000 in in vitro gene transfection tests of human cancer cells (Hela and MCF-7). Moreover, the presence of serum or addition of arginine during incubation of CSOSA-g-PEI micelles with plasmid DNA could further enhance gene transfection of CSOSA-g-PEI/pDNA. Besides, CSOSA-g-PEI conjugate highly localized in the tumor tissue and indicated a persistently increased accumulation (Hu et al., 2013).

\section{Tissue engineering}

Tissue engineering needs good biomaterials to serve as a temporary matrix and they must be able to afford porous forms providing channels for the migration of host cells into the matrix allowing complete tissue formation (Khor and Lim, 2003). Attempts have been made to use chitin as scaffolds in tissue engineering due to its wound healing, antibacterial, and anti-inflammatory properties (Maeda et al., 2008). However, due to its poor mechanical properties, biomaterials like hydroxyapatite (HAp) (Wang et al., 1997), bioactive glass ceramic (BGC) (Peter et al., 2009), silicon dioxide $\left(\mathrm{SiO}_{2}\right)$, titanium dioxide $\left(\mathrm{TiO}_{2}\right)$ and zirconium oxide $\left(\mathrm{ZrO}_{2}\right)$ (Deepthi et al., 2016) are used as hard tissue substitutes. Another interesting application is the hydroxyapatite-chitin-chitosan composite as bone-filling material for guided tissue regeneration for the treatment of periodontal bony defects, forming a self-hardening paste (Ito et al., 1998). Scaffolding strategies dealing with the osteochondral repair is a challenge for present-day research since it necessitates the combining of both bone and cartilage tissue engineering principles (Mano and Reis, 2007). A more recent study by Malafaya and Reis (2009) demonstrated that chitosan-based bilayer scaffolds could serve as alternative, biocompatible and safe biodegradable scaffolds for osteochondral (bone-cartilage) tissue engineering applications. In addition, more detailed applications of chitosan-chitin, chitin or chitosan/nHAp, chitin or chitosan/nBGC, chitin or chitosan $/ \mathrm{nSiO}_{2}$, chitin or chitosan $/ \mathrm{nTiO}_{2}$ and chitin or chitosan $/ \mathrm{nZrO}_{2}$ in bone regeneration could be found in articles by Deepthi et al. (2016), Liu et al. (2016) and Pangon et al. (2016).

\section{Anti-tumor activity}

Chitin and chitosan oligomers (COS) are known to have antitumor activities (Suzuki et al., 1986; Zhang et al., 2006; Azuma et al., $2015 b)$. COS ( $3 \mathrm{kDa}<\mathrm{MW}<5 \mathrm{kDa}$ ) was suggested to have chemopreventive activity in colon cancer by increasing the activities of quinone reductase (QR), and glutathione-S-transferase (GST), enhancing glutathione (GSH) levels, and inhibiting ornithine decarboxylase (ODC) activity and cyclooxygenase (COX)-2 expression in HT-29 cells (Nam et al., 2007). Shen et al. (2009) discovered that in vitro COS significantly inhibited cell proliferation, reduced the percentage of cells in S-phase, and decreased the rate of DNA synthesis in the HepG2 cells. Chitin and 6-O-carboxymethylchitin activate peritoneal macrophages in vivo, suppress the growth of tumor cells in mice and stimulate non-specific host resistance against Escherichia coli infection (Nishimura et al., 1984). Wang et al. (2003a, 2003b) reported that $\mathrm{N}$-acetyl glucosamine (NAG), D-glucosamine hydrochloride $\left(\mathrm{GlcNH}_{2} \cdot \mathrm{HCl}\right)$ and D-glucosamine $\left(\mathrm{GlcNH}_{2}\right)$ could induce proliferation of leukemia K562 cells and make them differentiate toward macrophage. $\mathrm{GlcNH}_{2}$ at a certain range concentration could kill tumor cells without influencing normal cells (Friedman and Skehan, 1980). It is therefore postulated that a combination of $\mathrm{GlcNH}_{2}$ with membrane-active drugs may have the potential to kill tumor cells, especially for neurooncology (Zhang et al., 2006). However, much controversy on the mechanism of antitumor activity of D-glucosamine and its derivatives still exists. Park et al. (2011) separated COS into different fractions and found that high molecular weight chitosan was approximately $50 \%$ less effective against cancer cell lines-PC3 (human prostate), A549 (human lung), and HepG2 (human hepatoma) compared to both COS.

\section{Fish bone minerals (calcium and phosphorus)}

Among fish processing by-products, fish bone serves as a potential source of calcium which is an essential element for human health (Kim and Mendis, 2006; Shahidi 2007). Bone, a composite tissue basically made up of two phases, an organic part, which comprises of collagen and non-collagenous protein, namely osteocalcin, osteopontin, osteonectin, fibronectin, thrombospondin, proteoglycan I, II and growth factors (IGF-1, PDGF and TGF- $\beta$ ) and an inorganic component (Garner et al., 1996; Jung et al., 2005). Despite the fact that fish bone contains bioactive and nutraceutical molecules, few efforts have been made to utilize fish bones in functional materials (Larsen et al., 2000; Kim et al., 2003; Jung et al., 2005; Jung and Kim, 2007). Figure 14 shows the calcium and phosphorus content of various fish bones. They were calculated according to the ratio of a mineral/total ash.

It is well documented that consumption of whole small fish is nutritionally beneficial by providing a rich source of readily absorbable calcium (Larsen et al., 2000). In Chinese and Korean markets, fish frame and fish bone have been developed as leisure snacks through various processing and formulation for more than 10 years (Kang et al., 2007, Li et al., 2008). However, very few information is available on the beneficial effects of larger fish bone and few attempts have been made to test their usage for benefiting human health. In order to incorporate fish bone into calcium-fortified foods, it is necessary to first convert it into an edible form by softening its structure. This could be achieved by hot water treatment and heat treatment in an acetic acid solution (Shahidi, 2007; Kim and Mendis, 2006), or by using enzymes (Liaset et al., 2003). Pepsin-assisted degradation of Alaska pollock bone in acetic acid solution led to highest degree of hydrolysis and dissolution of both mineral and organic parts of fish bone (Jung et al., 2005). Most recently, Tang et al. (2018) used grass fish bones as the substrate for treatment with Flavourzyme which was then fermented with Leuconostoc mesenteroides. The fermented solution so obtained had a high content of soluble calcium which was used to feed calcium-deficient rats. This calcium solution exhibited a high calcium bioavailability so that serum calcium, alkaline phosphatase levels, femur weight and other indicators in calcium-deficient rats could be returned to the normal levels.

\subsection{Application of fish bone minerals}

\subsubsection{Application of fish bone minerals in functional food/nutra- ceutical industry}

Calcium is required for numerous functions including strengthening of teeth and bones, nerve function and many enzymatic reactions 


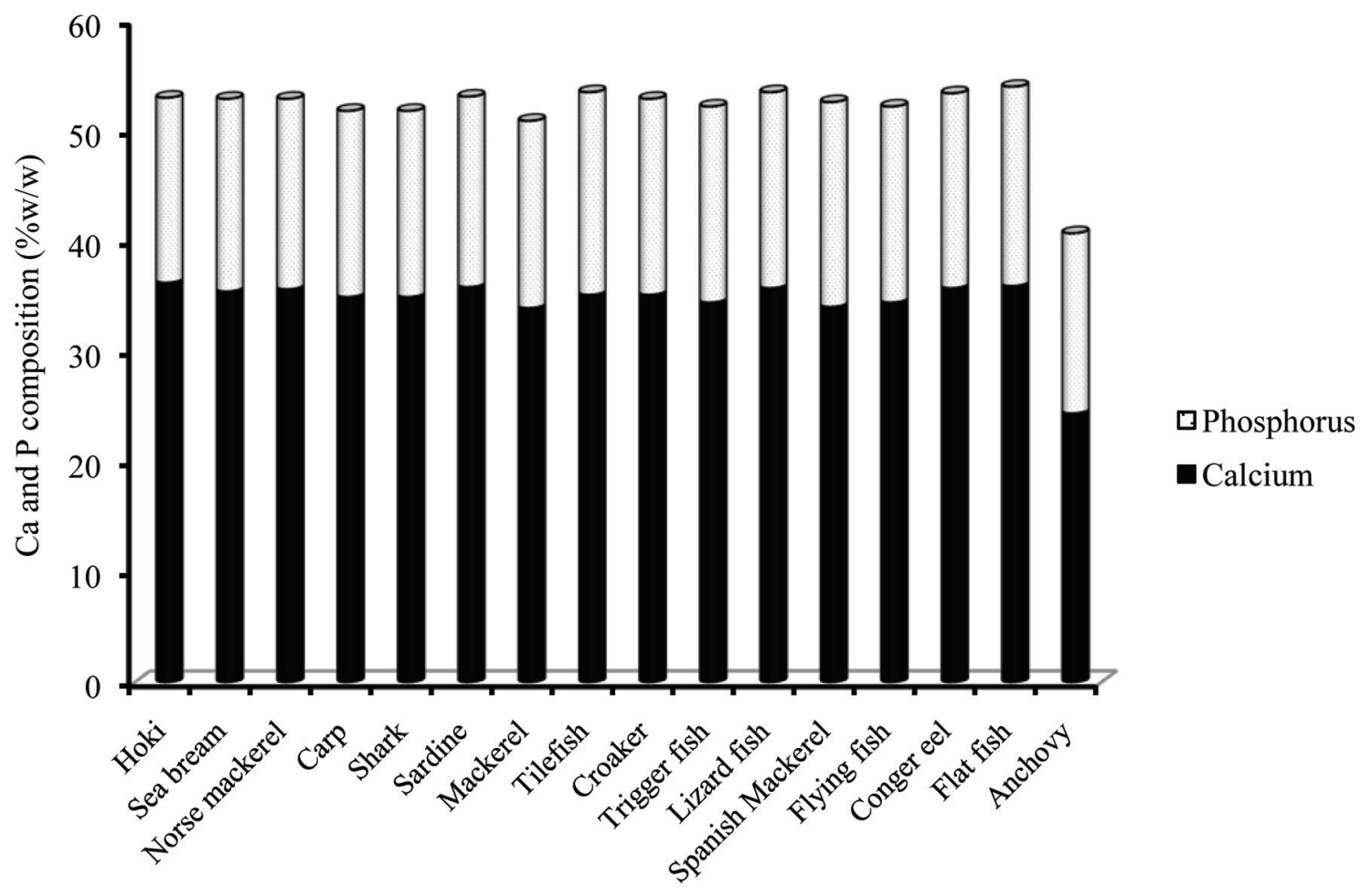

Figure 14. Ca and P composition in various fish bones. Source: Adapted from Hamada et al. (1995).

that require calcium as a co-factor (Kim and Jung, 2007). Generally, the most common and trusted source of calcium is milk (1cup serves $300-450 \mathrm{mg}$ ) and other dairy products (Garner and Anderson, 1996). However, alternative sources of $\mathrm{Ca}$ are needed, especially for population groups with a low intake of milk and dairy products and for those who do not drink milk due to lactose indigestion and intolerance. In milk and dairy products, casein phosphopeptides have the capacity to chelate $\mathrm{Ca}$ and to prevent the precipitation of $\mathrm{Ca}$ phosphate salts (Berrocal et al., 1989), thereby increasing the bioavailability (Yuan and Kitts, 1991, 1994). Similar to casein phosphopeptides, fishbone phosphopeptides could inhibit the formation of insoluble calcium phosphate and increase the bioavailability. In fish bone, calcium-binding phosphoproteins derived from non-collagenous material have a high affinity for $\mathrm{Ca}^{2+}$ on the surface hydroxyapatite (Hoang et al., 2003). Furthermore, a study by Jung et al. (2006) in rat models showed that fishmeal prevented $\mathrm{Ca}$ deficiency due to increased $\mathrm{Ca}$ bioavailability by the intake of fishbone phosphopeptides. Malde et al. (2010) later conducted a double-blind randomized crossover trial and found that calcium from enzyme-treated salmon and cod bones was well absorbed by healthy young men. Besides, it was also reported that fortified tuna bone powder supplementation increased bone mineral density and improved the maternal bone microstructure of lactating rats and their offsprings (Suntornsaratoon et al., 2018). Therefore, it is possible to provide a novel nutraceutical with high bioavailability for $\mathrm{Ca}$ and $\mathrm{Ca}$-fortified food supplements, such as fruit juice or $\mathrm{Ca}$-rich foods, and as alternatives to dairy products (Jung et al., 2006; Nemati et al., 2016).

\subsubsection{Application of fish bone minerals in biomedical industry}

Fish bone contains hydroxyapatite which unlike other calcium phosphates does not break under physiological conditions and takes part in bone bonding (Ozawa and Suguru, 2002). Hydroxyapatite $\left[\mathrm{Ca}_{10}\left(\mathrm{PO}_{4}\right)_{6}(\mathrm{OH})_{2}\right]$ has been introduced as a bone graft material in a range of medical and dental products due to similar chemical composition, biocompatibility and the ability to act as an osteoconductive/osteoinductive material (Gatti et al., 1990; Kitsugi et al., 1993; Zambonin and Grano, 1995; van Haaren et al., 2005; Best et al., 2008). Attempts have been made to isolate fish bone derived hydroxyapatite and use it as an alternate for synthetic hydroxyapatite (Kim et al., 1997; Ozawa and Suguru, 2002). For example, Shi et al. (2018) reported that among natural hydroxyapatite derived from rainbow trout, cod, and salmon bones, the ones from rainbow trout and salmon bones led to a faster growth, adhesion, differentiation, and proliferation of osteoblast and formation of mineralized tissue which is most likely attributed to the presence of $\mathrm{CO}_{3}{ }^{2-}$ and $\mathrm{Mg}^{2+}$. Generally, heat treatment $\left(800 \sim 1,300{ }^{\circ} \mathrm{C}\right)$ is used for the isolation of hydroxyapatite from fish bone and high temperature gives high strength and higher degree of crystallinity and larger crystallite size to hydroxyapatite structure (Choi et al., 1999; Pal et al., 2017; Sunil and Jagannatham, 2016) and provides an excellent biocompatible inorganic substance (Kim et al., 1998, 2001; Kim and Park, 2000; Venkatesan et al., 2015).

\section{Carotenoids}

Color plays a major role in the overall acceptability of food products (Shahidi and Brown, 1998). Crustacean processing discards provide a potential sources of carotenoids mainly astaxanthin (Shahidi and Brown, 1998). Increasing interest is being shown in astaxanthin and astaxanthin-containing raw materials for use in dietary formulation where integument and flesh coloration is of 
<smiles>CC1=CCCC(C)(C)C1/C=C/C(C)=C/C=C/C(C)=C/C=C/C=C(C)/C=C/C=C(C)/C=C/C1=C(C)CCCC1(C)C</smiles>

a<smiles>CC1=C(/C=C/C(C)=C/C=C/C(C)=C/C=C/C=C(C)/C=C/C=C(C)/C=C/C2=C(C)CCCC2(C)C)C(C)(C)CCC1</smiles>

b<smiles>CC1=CCCC(C)C1/C=C/C(C)=C/C=C/C(C)=C/C=C/C=C(C)/C=C/C=C(C)/C=C/C1C(C)=CCCC1(C)C</smiles>

C<smiles>CC(C)=CCC/C(C)=C/C=C/C(C)=C/C=C/C(C)=C/C=C/C=C(C)/C=C/C=C(C)/C=C/C=C(\C)CCC=C(C)C</smiles>

Figure 15. The chemical structures of $\alpha$-carotene (a), b-carotene (b), $\varepsilon$-carotene (c), and lycopene (d).

economic importance (Shahidi and Brown, 1998). Carotenoids are among the main natural food colorants with widespread use. The extracted carotenoids from shrimp waste would be a cheaper alternative than synthetic carotenoids in aquaculture feed formulations and in surimi-based products (Sachindra et al., 2006).

Carotenoids are red, yellow, and orange pigments and are synthesized only in plants. However, they are widely distributed in nature, almost in all living matters (Buchecker, 1982). More than seven hundred naturally occurring carotenoids have been identified, of which as many as fifty may be absorbed and metabolized by the human body (Goodwin, 1994). Although it is generally accepted that animals are unable to synthesize carotenoids de novo, they apparently are able to modify dietary plant carotenoids $(\mathrm{Bu}-$ checker, 1982).

Chemically, carotenoids can be divided into two major classes. The first class contains highly unsaturated hydrocarbon carotene $\left(\mathrm{C}_{40} \mathrm{H}_{56}\right)$ such as lycopene, $\alpha$-carotene, $\beta$-carotene, and $\varepsilon$-carotene, which have no oxygen and are usually orange and red in color and are soluble in organic solvents such as petroleum ether (Figure 15) (Krinsky, 1994). Because they are highly unsaturated, they are particularly susceptible to oxidation. The second class contain oxygen and are known as xanthophylls; in these, the oxygenated group substituents occur at particular sites on the terminal rings (Krinsky, 1994; Shi and Maguer, 2000; Young and Lowe, 2001). The most frequently found groups are hydroxy- (monols, diols, and polyols), epoxy- (5,6- and 5,8- epoxides), methoxy, aldehyde, oxo, carboxy, and ester moieties (Figures 16 and 17) (Gross, 1991).

Carotenoids are among the most important natural marine pig- ment groups (Matsuno and Hirao, 1989). The ovaries and eggs of fish and shellfish are almost always pigmented. The colors range from yellow, orange and red to green, blue and purple, mainly due to the presence of carotenoids and/or carotenoproteins (Miki et al., 1982). Carotenoproteins are the complex formed by the binding of carotenoids with hydrophobic sites of proteins. They may cause the pigment to become water-soluble, and it may change the color of the pigment (Shahidi and Brown, 1998). Crustacean possesses three main types of pigments namely, carotenes, xanthophylls, and conjugated carotenoids, which are mainly carotenoproteins, carotenolipoproteins, and the chitinocarotenoids. Carotenolipoproteins such as lipovitellins, mainly found in the ovaries and eggs, are responsible for the blue, green, or purple color, and chitinocarotenoids occur mainly in the exoskeleton (Ghidalia, 1985). The real carotenoproteins such as crustacyanin are usually present on the external surfaces of crustacea and account for their vivid coloration. Crustacyanin is almost always astaxanthin, which is attached at specific sites to a (glyco) protein (Shahidi and Brown, 1998). Cleavage of the complex results in a color change caused by the liberation of the free carotenoids (Nelis et al., 1989). The principal carotenoids in crustacea include beta-carotene, echinenone, canthaxanthin, two stereoisomers of phoenicoxanthin, three stereoisomers of astaxanthin, 4-ketozeaxanthin, fritschiellaxanthin, and papilioerythrinone (Figure 18) (Shahidi and Brown, 1998).

Carotenoids can be recovered from shellfish processing byproducts and used in a variety of applications (Shahidi and Brown, 1998). Shrimp processing industries generate large quantities of shrimp waste in the form of head and body carapace, which 
<smiles>CC1=C(/C=C/C(C)=C/C=C/C(C)=C/C=C/C=C(C)/C=C/C=C(C)/C=C/C2=C(C)C[C@@H](O)CC2(C)C)C(C)(C)CCC1</smiles>

a<smiles>CC1=C[C@@H](O)CC(C)(C)[C@H]1/C=C/C(C)=C/C=C/C(C)=C/C=C/C=C(C)/C=C/C=C(C)/C=C/C1=C(C)C[C@@H](O)CC1(C)C</smiles>

b<smiles>CC1=C(/C=C/C(C)=C/C=C/C(C)=C/C=C/C=C(C)/C=C/C=C(C)/C=C/C2=C(C)CC(O)CC2(C)C)C(C)(C)CC(O)C1</smiles>

C<smiles>CC1=CC(=O)CC(C)(C)/C1=C/C=C(C)/C=C/C=C(C)/C=C/C=C/C(C)=C/C=C/C=C(C)/C=C/C1=C(C)CC(=O)CC1(C)C</smiles>

d<smiles>COC(C)(C)C/C=C/C(C)=C/C=C/C=C/C(C)=C/C=C/C(C)=C/C=C/C=C(C)/C=C/C=C(C)/C=C/C=C(\C)CCCC(C)(C)O</smiles>

e

Figure 16. The chemical structures of cryptoxanthin (a), lutein (b), zeaxanthin (c), rhodoxanthin (d) and (e) rhodovibrin.

comprise $45-60 \%$ of the whole shrimp (Barratt and Montano, 1986). The carapace as well as the ecdysial exoskeleton contain mainly free astaxanthin and lutein (Castillo et al., 1982). The oil solubilization characteristics of carotenoids have led to studies on recovery of these pigments in oils from crustacean wastes (Anderson, 1975, Spinelli and Mahnken, 1978, Chen and Meyers, 1982, Shahidi and Synowiecki, 1991 and Sachindra and Mahendrakar, 2005). Sachindra et al. (2005) extracted the carotenoids from Indian shrimp waste with contents varying from 35 to 153 $\mu \mathrm{g} / \mathrm{g}$ depending on the species; the major pigment being astaxanthin and its esters. Similarly, Shahidi and Synowiecki (1991) isolated astaxanthin and astaxanthin esters and zeaxanthin from shrimp Pandalus borealis and astacene, canthaxanthin, and lutein from processing discards of snow crab Chinoecetes opilio. More recently, Takeungwongtrakul et al. (2015) extracted carotenoids accompanied with lipid from hepatopancreas of Pacific white shrimp (Litopenaeus vannamei). Among single solvents (acetone, isopropanol and hexane) and their mixtures, a mixture of hexane and isopropanol $(50: 50, \mathrm{v} / \mathrm{v})$ rendered lipids with the highest carotenoid yield $(336.40 \mathrm{mg} / \mathrm{kg}$ hepatopancreas) with the extraction yield of $18.22 \%$ (w/w hepatopancreas). The addition of astaxanthin $(2 \mathrm{mg} / \mathrm{g}$ lipid) into the lipid could lower lipid oxida- tion, in comparison with the control during storage at $30{ }^{\circ} \mathrm{C}$ for 10 days as indicated by a lower $p$-anisidine value. Other organic solvents such as acetone (Mandeville et al., 1991; Masatoshi and Junji, 1999; Sachindra et al., 2005b) and alcohol (Kozo, 1997) were also examined for the recovery of carotenoid pigments. $\mathrm{Li}$ et al. (2005) extracted carotenoids from fish eggs using acetone, and the carotenoids in acetone extract were phase separated by using methyl tertiary-butyl ether. Charest et al. (2001) used alcohol as co-solvent in supercritical $\mathrm{CO}_{2}$ extraction of astaxanthin from crawfish shells. However, along with organic solvents, different auxiliary techniques including microwave-, ultrasonic- and enzyme-assisted extractions were commonly used to increase the extraction efficiency and carotenoids recovery (Martins and Ferreira, 2017). Selective extraction of astaxanthin from crustacean waste has been attempted by supercritical carbon dioxide extraction technique (Lopez et al., 2004). Although the use of organic solvents for recovery of carotenoids from shrimp waste has been limited to analytical purposes only (Britton, 1985; Meyers and Bligh, 1981), some of the organic solvents permitted for use in food industries include acetone, benzyl alcohol, ethyl acetate, hexane, isopropanol, methanol, methyl ethyl ketone, and ethanol, However, levels of use depends on the type of foods in which they 


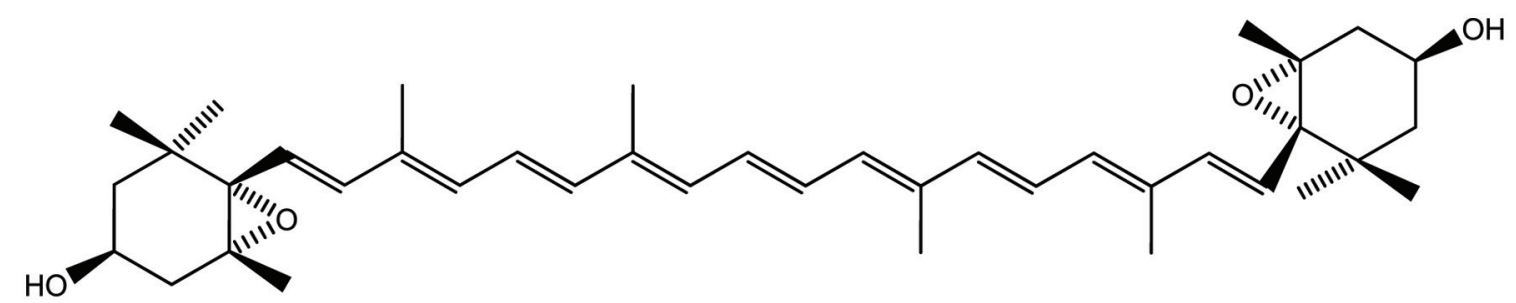

a<smiles>COC(=O)/C=C/C(C)=C\C=C\C(C)=C\C=C\C=C(C)\C=C\C=C(C)\C=C\C(=O)O</smiles><smiles>CC(/C=C/C=C(C)/C=C/C(=O)O)=C\C=C\C=C(C)\C=C\C=C(/C)C(=O)O</smiles>

c

Figure 17. The chemical structures of violaxanthin (a), bixin (b), crocetin (c).

are used (Food and Drug Regulation, 2005).

It has been reported that the stability of carotenoids in shrimp by-products is affected by the method of preservation (Sachindra et al., 2007). Tanaka et al. (1981) observed that carotenoid content in Antarctic krill meal was decreased during storage at room temperature. Therefore, the addition of antioxidants has been suggested to prevent pigment degradation during storage of crawfish meal (Meyers and Bligh, 1981 and Chen and Meyers, 1982). In addition to this, ensiling in organic/inorganic acids or by fermentation is used as one of the methods to preserve the shrimp waste (Ariyani and Buckle, 1991; Sachindra et al., 2007).

\subsection{Application of carotenoid pigments}

The most important factor affecting the consumer acceptance of cultivated fish (salmonids) is the color of its flesh, and in the case of crustaceans the color of the carapace is a determining factor (Shahidi and Brown, 1998). Marine fish flesh and shellfish appear rich in color due to carotenoids, which are obtained through the diet by mainly consuming zooplankton and nekton. Therefore, cultivated fish species require adequate pigmentation to respond to consumer's demands. Moreover, adequate pigmentation is also important to meet the customer's expectation (Shahidi and Brown, 1998). In salmon and crustacean culture systems, it is necessary to use dietary astaxanthin in order to achieve flesh pigmentation because natural pink color of the salmon fish and the red color of boiled crustacean shells are due to the carotenoid astaxanthin (Shahidi and Brown, 1998). Meanwhile, Wang et al. (2018) found that dietary carotenoids could not only improve body color of farmed crab, but also protect them from oxidative damage caused by high $\mathrm{pH}$ environment. Therefore, the by-products from shrimp and crab have been established as a good source of astaxanthin for salmon and crustacean culture systems (Simpson, 1982; Torrissen et al., 1982; Meyers, 1977). In addition to the application in aquaculture, carotenoids have also been incorporated in poultry feed to impart desirable coloration to egg yolks of various poultry animals (Suresh et al., 2018). The global market for carotenoid pigments has increased from 766 million US dollar (2007) to 1.5 billion US dollar (2017) in 10 years (McWilliams, 2018; Kirti et al., 2014). Therefore, increasing interest is being shown in astaxanthin and astaxanthin-containing raw materials for use in dietary formulation where integument and flesh coloration is of economic importance. Furthermore, astaxanthin can be used as a colorant in food and in medicine due to its attractive orange color, its biological functions as a vitamin A precursor and antioxidative activity (Cremades et al., 2001; Stepnowski et al., 2004; Shahidi and Brown, 1998). Mention should also be made that canthaxanthin has also been used for coloration of slamonif fish flesh.

\section{Seafood flavor}

The production of natural seafood flavor extracts from processing by-products has been an industrial practice in France and Japan. As a common industrial practice, the natural seafood flavors are reformulated by adding other ingredients and artificial flavors for characteristics desired (Lee, 2007). Seafood flavors are being used in seafood sauces, chowders, soups, bisques, instant noodles, snacks, 
and surimi (Lee, 2007). Generally, three basic process routes exist for making seafood flavors from finfish and shellfish processing by-products. These include aqueous extraction, fermentation and enzymatic hydrolysis (Lee, 2007). However, enzymatic hydrolysis is mostly employed in commercial seafood flavor extract preparations because it has a significant impact on the volatile compound profile (Lee, 2007). Free amino acids and peptides act as precursors of the volatile compounds, which are formed through Maillard reaction. According to Hayashi et al. (1981) and Izzo and Ho (1992), each amino acid and peptide makes its specific contribution to the volatile compound profile. Konosu (1979) and Hayashi et al. (1981) found that Glu, Gly, Arg, AMP and GMP are the primary taste-active components in snow crab. The taste-active components in clam were Glu, Gly, Arg, Tau, AMP, and succinic acid (Fuke et al., 1989). Kim et al. (2003) demonstrated that shrimp by-products such as heads, shells, and tails may serve as a potential source of flavorants such as shrimp sauce. Nowadays most successful products in the market are clam, crab, shrimp, and lobster extracts which could be produced from shellfish processing byproducts (Lee, 2007). In addition, whole or process by-products of finfish can be utilized for production of fish flavor extracts (Lee 2007). Figure 19 shows the flow chart of clam flavor production from sea clam processing by-products.

Imm and Lee (1999) showed that protein hydrolysate prepared from red hake had a great potential as a fish flavorant that could be used as a flavor supplement and sauce for various seafood products. Furthermore, they suggested that a good quality fish flavor can be produced from unutilized frame waste. The production of seafood flavors from underutilized fish species through protein hydrolysis is somewhat challenging due to the difficulty of ensuring high organoleptic quality (Imm and lee, 1999). The hydrolysis of protein often accompanies flavor defects such as bitterness and off-flavor along with the formation of desirable flavor (Lee, 2007; Kilara, 1985). To be effective in hydrolyzing and debittering, the enzyme should contain both endoprotease for hydrolysis of protein and exopeptidase for breaking down bitter peptides (Lee, 2007). For example, in the seafood flavor formulations, Peinado et al. (2016) used a combination of endo- and exo-peptidases to increase the concentration of free amino acids, particularly leucine, lysine, and glutamic acid in the final protein hydrolysates of fish by-products. Meanwhile, an acidic serine carboxypeptidase isolated from the hepatopancreas of squid was able to eliminate the bitterness by removing specific amino acids in the carboxyl terminal (Komai et al., 2007). In addition, flavor quality of hydrolysate depends on the quality of raw material, which plays a critical role in determining flavor quality (Imm and Lee, 1999). Fatty fish species are not desirable because of their high susceptibility to lipid oxidation and the high cost of removing excess fat (Ritchie and Mackie, 1982). The extent of hydrolysis determines sensory quality and is dependent upon the specificity of protease, level of the enzyme, water-to-substrate ratio, $\mathrm{pH}$, and temperature (Imm and Lee, 1999). In a recent article, Ambigaipalan and Shahidi (2017) reported the preparation of hydrolysates from shrimp processing by-products but did not comment on the flavor profile of the product so prepared.

\section{Enzymes}

Enzyme technologies have become an important part of the processes used by the modern food and feed industry to produce a large and diversified range of products (Shahidi and Kamil, 2001). In addition, enzyme-catalyzed processes have been successfully introduced to other industries such as pharmaceuticals and agrochemicals, but now also increasingly used in organic chemical synthesis because they exhibit a number of features that make their use advantageous as compared to conventional chemical catalysts (Krajewska, 2004). Foremost among them are a high level of catalytic efficiency, often far superior to chemical catalysts, and a high degree of specificity that allows them to discriminate not only between reactions but also between substrates (substrate specificity), similar parts of molecules (regiospecificity) and between optical isomers (stereospecificity) (Krajewska, 2004).

Marine enzymes have drawn the attention of numerous researchers and there are several excellent reviews on the utilization of enzymes from aquatic organisms (Morrissey and Okada, 2007; Shahidi and Kamil, 2001; Gildberg et al., 2000; Haard, 1998). Aquatic organisms include a wide and extensive taxonomic diversity and many organisms occupy unusual environmental habitats, thus conferring to enzymes some unique characteristics (Shahidi and Kamil, 2001; Guérard et al., 2005). The glands and digestive organs such as stomach, pyloric cecum, pancreas, and intestines are the most important by-products of fish and shellfish processing in terms of the quantity of enzymes present (Kurtovic et al., 2009; Nayak et al., 2003).

The most extensively studied enzymes from the marine environment include pepsin, trypsin, chymotrypsin, elastase, collagenase and alkaline phosphatase isolated from Atlantic cod (Gadus morhua) viscera (Simpson and Haard, 1984a; Simpson and Haard, 1984b; Gildberg 1992), polar cod (Boreogadus saida) (Arunchalam and Haard, 1985), dogfish (Guérard and Gal 1987 and 1989; Hajjou et al., 1995), salmon (Haard et al., 1996; Kristinsson and Rasco, 2000) and tropical tuna (Smine et al., 1993). Several of these enzymes from poikilothermal organisms are cold active and have a catalytic activity equal to or higher than mammalian enzymes (Krajewska, 2004).

Among naturally available enzymes from fish and aquatic invertebrates, proteases constitute a major group; these have been referred to as proteinases (endo-) and peptidases (exo-). Exopeptidases cleave peptide bonds at the amino or carboxyl ends of the polypeptide chain, whereas endopeptidases cleave internal peptide bonds. These terms refer to all enzymes, which catalyze the hydrolysis of peptide bonds in polypeptides and proteins (Shahidi and Kamil, 2001). Proteases from various sources differ greatly in their catalytic and physical properties (Han and Shahidi, 1995). Digestive proteolytic enzymes include pepsin, trypsin, chymotrypsin, elastase, gastricin and others (Morrissey and Okada, 2007). Regardless of the source, proteases can be classified based on the well-known characterized proteases, such as trypsin-like, chymotrypsin-like, chymosin-like, or cathepsin-like (Sriket, 2014). Besides, they may also be classified depending on their substrate specificity, including serine/cysteine/aspartic proteases, and metalloproteases or sensitivity of $\mathrm{pH}$, including acid, neutral or alkaline proteases (Sriket, 2014). Fish intestines are known to be the richest source of various enzymes including chitinase, alkaline phosphatase, and hyaluronidase, followed by pyloric ceca, pancreatic tissues, hepatopancreas, shell, and other waste components (Shahidi and Kamil 2001; Venugopal, 2016). Some isolation processes for enzymes include precipitation by salts and polyacrylic acids, isoelectric solubilization/precipitation, ultrafiltration, $\mathrm{pH}$ shift, flocculation and membrane filtration as well as overcooled acetone extraction (Venugopal, 2016). Reece (1988) described a suitable method for large-scale recovery of proteolytic enzymes from fish viscera silage. Balti et al. (2009) reported a five-step method including fractionation with ammonium sulfate, Sephadex G-100 gel filtration, DEAE-cellulose an ion-exchange chromatography, Sephadex G-75 gel filtration, and Q-Sepharose anion-exchange 
<smiles>CC1=C(/C=C/C(C)=C/C=C/C(C)=C/C=C/C=C(C)/C=C/C=C(C)/C=C/C2=C(C)C(=O)C(O)CC2(C)C)C(C)(C)CCC1=O</smiles>

\section{Phoenicoxanthin}<smiles>CC1=C(/C=C/C(C)=C/C=C/C(C)=C/C=C/C=C(C)/C=C/C=C(C)/C=C/C2=C(C)C(=O)[C@@H](O)CC2(C)C)C(C)(C)C[C@H](O)C1</smiles>

\section{4-Ketozeaxanthin}<smiles>CC1=C[C@@H](O)CC(C)(C)[C@H]1/C=C/C(C)=C/C=C/C(C)=C/C=C/C=C(C)/C=C/C=C(C)/C=C/C1=C(C)C(=O)[C@@H](O)CC1(C)C</smiles>

Frits-chiellaxanthin<smiles>CC1=CC(=O)CC(C)(C)C1/C=C/C(C)=C/C=C/C(C)=C/C=C/C=C(C)/C=C/C=C(C)/C=C/C1=C(C)C(=O)C(O)CC1(C)C</smiles>

\section{Papilioerythrinone}<smiles>CC1=C(/C=C/C(C)=C/C=C/C(C)=C/C=C/C=C(C)/C=C/C=C(C)/C=C/C2=C(C)C(=O)[C@@H](O)CC2(C)C)C(C)(C)C[C@H](O)C1=O</smiles>

Astaxanthin

Figure 18. The chemical structures of principal carotenoids in crustaceans.

chromatography to purify thermostable trypsin from the hepatopancreas of Sepia officinalis.

Fish pepsins belong to the aspartic endopeptidase family and are digestive proteases, which are normally located within the fish stomach and have peak activity under acidic conditions (Morrissey and Okada, 2007). However, it has been found that many fish species secrete at least two different pepsins with different $\mathrm{pH}$ optima
(Gildberg, 1988; Gildberg and Raa, 1983); these are generally recognized as pepsin I and pepsin II (Gildberg, 1988). Fish pepsins have been isolated from the gastric mucosa of various marine and freshwater fish species, including those from cold- and warm-water species (Shahidi and Kamil 2001). Trypsin can be categorized as serine proteinase and it has a dual role in that it cleaves ingested proteins and activates the precursor forms of several other diges- 


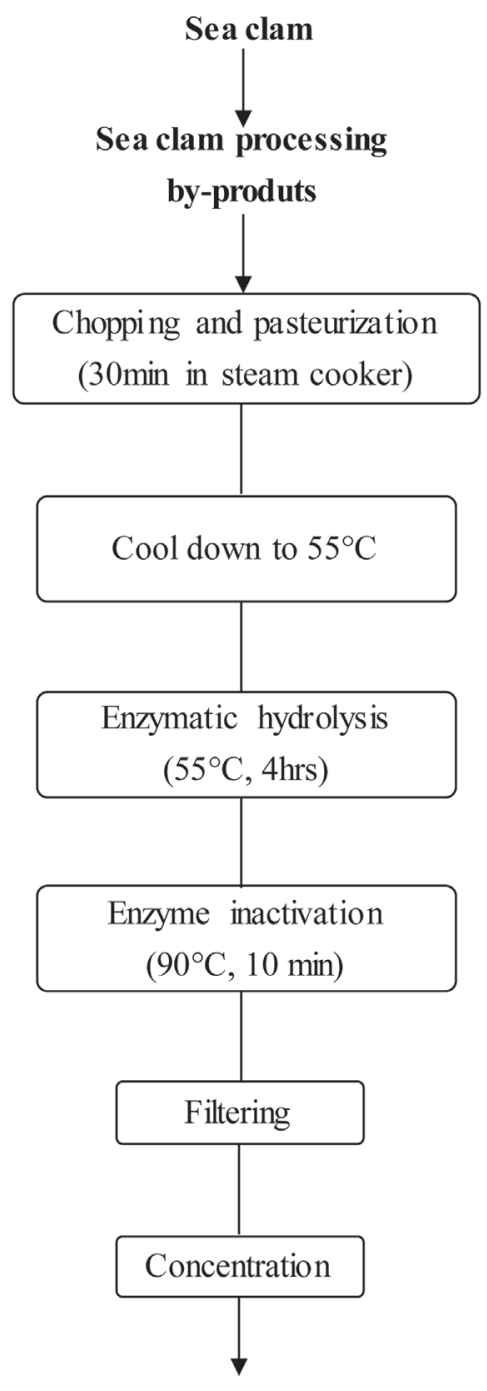

Clam flavor extracts

Figure 19. Preparation of clam flavour from clam processing by-product. Source: Adapted from Lee (2007).

tive proteinases including chymotrypsin (Morrissey and Okada, 2007). Trypsin is normally located in pyloric cecum and shows its activity at neutral and alkaline conditions (Morrissey and Okada, 2007). Trypsin and chymotrypsin have been isolated and identified in a wide array of cold water as well as warm water fish species (Shahidi and Kamil 2001).

Chymosin (EC 3.4.23.4), commonly known as 'rennin', is an acidic protease (Wong, 1995) which shows stability near $\mathrm{pH} 7.0$ for proteolysis and has a narrow substrate specificity. Chymosin and chymosin-like enzymatic activities have been reported in carp (Cohen and Gertler, 1981), and harp seal stomach (Han and Shahidi, 1995; Shamsuzzaman and Haard 1983; Shamsuzzaman and Haard, 1984). Gastricsins are aspartyl proteases that possess similar enzymatic and chemical properties to pepsin (De-Vecchi and Coppes, 1996; Sanchez-Chiang and Ponce, 1981). Two zymogens of gastricsin from the stomach of hake (Merluccius gayi) have been extracted by Sanchez-Chiang and Ponce, (1981) who reported that both zymogens were active up to $\mathrm{pH} 10$ with optimal activity at $\mathrm{pH}$ 3.0.

\subsection{Collagenases and elastases}

Collagenases are generally defined as enzymes that are capable of degrading the polypeptide backbone of native collagen under conditions that do not denature the protein (Kim et al., 2002). They could be divided into serine collagenases and metallocollagenases and they are thought to play different physiological functions (Kim et al., 2002).

Collagenolytic serine proteases have been isolated and characterized from digestive tracts of various fish and aquatic invertebrates, including fiddler crab (Elsen and Jeffrey, 1969; Grant et al., 1983), fresh water prawn (Baranowski et al., 1984), crayfish (Garcia-Carreno et al., 1994), king crab (Gerasimova and Kupina, 1996; Zefirova et al., 1996) and Atlantic cod (Kristjansson et al., 1995). These enzymes are most active in the $\mathrm{pH}$ range of $6.5-8.0$ and are inactivated at $\mathrm{pH}$ values lower than 6.0 (Haard and Simpson, 1994). On the other hand, the collagenase fraction from mackerel (Scomber japonicus) was shown to be optimally activated at $\mathrm{pH} 7.5$ and $55^{\circ} \mathrm{C}$, while the metalloproteases with molecular masses of 64,67 and $75 \mathrm{kDa}$ were found in the dark muscle of common carp with optimal $\mathrm{pH}$ at 7-9 and in the presence of calcium (Park et al., 2002; Sriket, 2014; Wu et al., 2008). Furthermore, Zefirova et al. (1996) reported that practical use of these enzymes is restricted due to their low heat stability; it has also been shown that such collagenases lose their activity even at $40^{\circ} \mathrm{C}$.

Pancreatic elastase belongs to the serine protease family (Asgeirsson and Bjarnason, 1993 and De-Vecchi and Coppes, 1996) and has the ability to digest the elastic, fibrous protein of the connective tissue known as elastin (Asgeirsson and Bjarnason, 1993). However, Shotton (1970) reported that elastase can digest a wide variety of other proteins in addition to native elastin. Gastric elastase enzymes have been recovered from marine and fresh water fish species such as carp (Cohen and Gertler, 1981), catfish (Clark, et al., 1985), and Atlantic cod (Asgeirsson and Bjarnason, 1993; Asgeirsson, et al., 1998; Gildberg and Overbo, 1990; Raa and Walther, 1989). Apart from the collagenases distributed in digestive tract, they were also found in fish muscle tissues including skeletal muscle of mackerel, Scomber japonicus, Japanese flounder, Paralichthys olivaceus, rainbow trout and common carp (Sriket, 2014). Additionally, gelatinolytic serine proteases possess similar properties to collagenase which have been proposed to participate in the metabolism of collagens and in the post mortem degradation of fish muscle during cold storage in species like red sea bream (Sriket, 2014, Wu et al., 2010). Felberg et al. (2010) partially characterized the peptide sequencing of gelatinolytic trypsin-like serine proteases, as well as other enzymes including elastase, trypsin, metalloproteases and aspartyl aminopeptidase. These endogenous proteases play a critical role in the degradation of fish and shellfish proteins, resulting in their tissue softening and compromising their shelf-life.

\subsection{Chitinolytic enzymes}

Chitinolytic enzymes, (true chitinases) are widely found in almost all living forms including bacteria, fungi, plants, insects, mammals and viruses, as well as being distributed in crustaceans (Islam and Datta, 2015). They play an important role in the degradation of chitin (Morrissey and Okada, 2007). Hydrolysis of chitin by chitinolytic enzymes leads to the production of monomers which are of tremendous industrial use such as in medicine (Islam and Datta, 2015). In addition, lysozymes exhibit chitinolytic activity (Clark et al., 1988). Chitinases can be divided into endo-type (chitinase) and 
Table 3. Digestive Lipases extracted from different fish species

\begin{tabular}{lll}
\hline Fish sp & Digestive organs/glands & Reference \\
\hline Atlantic cod (Gadus morhua) & pyloric ceca/pancreas & Gjellesvik et al. (1994) \\
Atlantic salmon (Salmo salar) & pancreas & Gjellesvik et al. (1994) \\
Red sea bream (Pagrus major) & hepatopancreas & lijima et al. (1998) \\
Rainbow Trout (Oncorhynchus mykiss) & Inter-cecal pancreatic tissue & Leger et al. (1977) \\
& Pyloric ceca & Tocher and Sargent (1984) \\
Oil sardine (Sardinella longiceps) & hepatopancreas & Mukundan et al. (1985) \\
Spiny dogfish (Squalus acanthius) & Pancreas & Rasco and Hultin (1988) \\
Nile Tilapia (Oreochromis niloticus) & Stomach, intestine & Taniguchi et al. (2001) \\
Grey Mullet (Mugil cephalus) & Viscera (pyloric ceca, intestines, and associated mesenteries) & Aryee et al. (2007) \\
\hline
\end{tabular}

exo-type ( $\beta$-N-acetylhexosaminidase) and are involved in molting in several crustaceans (Kono et al., 1995). These enzymes have been purified mainly from processing by-products such as squid liver (Matsumiya, 2007), shrimp waste silage (Matsumoto et al., 2004), and shrimp by-products (Olsen et al., 2014). Chitinolytic enzymes are a prime tool for converting chitin into oligomeric units without the use of the chemical depolymerization (Shahidi and Kamil, 2001). Furthermore, these enzymes may be used as bacteriolytic enzymes mainly due to their ability to degrade peptidoglycan components of cell walls of bacteria (Shahidi and Kamil, 2001; Stoykov et al., 2015).

\subsection{Lipases}

Lipases are a broad family of enzymes that catalyze the hydrolysis of ester bonds in substrates such as triacylglycerols, phospholipids, cholesteryl esters, and vitamin esters (Wong and Schotz, 2002). Within the above classification, the so-called "true lipase" or acylglycerol acyl hydrolase (E.C. 3.1.1.3) is defined as the enzyme which hydrolyzes esters of long-chain aliphatic acids from glycerol at the oil/water interface (Jensen, 1983). Lipases share the common characteristics of the optimum temperature of less than $35^{\circ} \mathrm{C}$, optimum $\mathrm{pH}$ ranging from neutral to slightly alkaline and low thermal stability (Kurtovic and Marshall, 2013). In the study of Cao et al. (2018), positive correlations existed between the lipolytic activity of endogenous lipase and lipid oxidation on the preservation of both traditionally salted fish and low-salt lactic acid-fermented fish. Therefore, lipases are receiving an increasing interest due to their effects on the quality of food products and their actual and potential applications in modified foods and industrial processes (Kurtovic et al., 2009). Particularly it is important to investigate the lipolytic enzymes originating from aquatic organisms for use in catalyzing interesterification of fish oils to produce triacylglycerols enriched with $\omega$-3 PUFA (Simpson et al., 1991).

One of the earlier investigations of Chesley (1934) in different marine species reported that active fishes, mackerel and scup, had markedly higher lipase activities than more sluggish species, with the upper intestine, pyloric ceca, and pancreas containing most of the enzyme. For long time research in fish digestive lipolysis was inconclusive as to which type (pancreatic lipase or carboxyl ester lipase) of lipases were present in fish (Leger et al., 1977; Patton, 1975; Patton et al., 1975). However, purification of first non-mammalian carboxyl ester lipase (CEL) from the pyloric ceca/pancreas of Atlantic cod by Gjellesvik et al. (1992) and cDNA sequence of pancreatic CEL from Atlantic salmon reported by Gjellesvik et al.
(1994), some new insights into the nature of fish digestive lipases were presented.

Commercially, Alaska pollock viscera is a potential source of useful lipases (McGinnis and Wood, 2007), especially as the diet of the fish contains a high percentage of phospholipids and wax esters (Lee et al., 1970; Saito et al., 2002). A project about utilizing valuable enzyme from Norwegian herring was mentioned by Kurtovic and Marshall (2013). Another possible large source of novel marine lipases is Antarctic krill which contains more than half of total storage lipids as wax esters (Ju and Harvey, 2004), suggesting the presence of possibly unique wax-ester specific lipases (Kurtovic et al., 2009). For more information about the use of lipases motivated by the valorisation of fish by-product, the reader may consult a review by Kurtovic and Marshall (2013). In addition, digestive lipases have been purified and characterized from various other aquatic organisms (Table 3 ).

Digestive lipases are mentioned in a few studies conducted on marine crustaceans, e.g., American lobster (Homarus americanus) larvae (Biesiot and Capuzzo, 1990). A few bivalves and a cephalopod have also been examined (Teo and Sabapathy, 1990; Itabashi and Ota, 1994).

\subsection{Applications of digestive enzymes from marine sources}

Cold-active fish pepsins from species such as Atlantic cod $(G$. morhua) and orange roughly (Hoplostetus atlanticus) have been used for caviar production from the roe of various species (Guérard et al., 2005). Furthermore, these proteolytic enzymes are used to ease the riddling process, increasing the yield from 70 to $90 \%$ for salmon (S. salar) (Guérard et al., 2005). The temperature optima of trypsin and alkaline phosphatase from cold-water fish are about $30{ }^{\circ} \mathrm{C}$ lower than the homologues from warm water fish or mammals (Simpson and Haard, 1987; Gelman et al., 1989). This property is advantageous in applications where it is desirable to inactivate the enzyme with mild heat treatment (Haard, 1998). Enzymes like lactate dehydrogenase (LDH) from deep-water fish that live at high pressure have a tighter polypeptide structure than homologues at normal atmospheric temperature, thus making them more resistant to proteolytic degradation and more suitable for applications where proteases might interfere with their activity (Hennessey and Siebenaller, 1987). The gastric proteases of fish that take in saltwater during feeding are salt-activated, in contrast to homologues from mammals that are inhibited by $\mathrm{NaCl}$. This property may be advantageous in applications such as fermentations, silage, and fish sauce where significant amounts of salt are pre- 
sent (Squires et al. 1986). Lysozyme from Artic scallop (Myrnes and Johansen, 1994) and other fish have a unique ability to attack both gram-positive and gram-negative bacteria (Guérard et al., 2005). Transglutaminase can be used in certain food products such as surimi, seafood, and meat products that require improved gel formation and gel strength resulting in better texture (Morrissey and Okada, 2007; Binsi and Shamasundar, 2012). Exogenous proteolytic enzymes such as squid hepatopancreases could be used in accelerating the production of capelin sauce (Venugopal, 2016). While endogenous proteolytic enzymes from fish may be used for skin removal from fish. This would serve as an alternative method to mechanical or chemical treatments, which often damage or reduce the recovery of the product (Haard and Simpson, 1994). Furthermore, proteases from marine sources can be used in the deskinning of squid (Shahidi and Kamil, 2001). Proteases from plant sources such as papain and bromelain are principally used for meat tenderization (Haard and Simpson, 1994). These enzymes attack both connective and myofibrillar proteins which often leads to over tenderization. Marine collagenase could only hydrolyze connective tissue protein possibly resulting in optimum tenderization of meat products (Morrissey and Okada, 2007). Furthermore, various proteins such as myofibrillar and sarcoplasmic proteins and collagen could be recovered from fishery processing wastes including fins, scales, skins, bones, head, and swim bladders through protease hydrolysis. The pepsin-solubilized collagen and its hydrolysate co-products could serve as potential substitutes to mammalian collagen used in food, pharmaceutical, and photographic industries (Venugopal, 2016). During various enzymatic processing, fish protein hydrolysates could be mainly/co-produced which exhibit diverse bioactivities including antioxidant, antimicrobial, antihypertensive, antiamnesiac, mineral binding, immunomodulatory, and antithrombotic functions. Thus with their diverse physicochemical properties, they can be used as food binders, emulsifiers, gelling agents, nutritional supplements, and biomedicals (Chalamaiah et al., 2012; Hernández-Ledesma and Herrero, 2013; Kim and Mendis, 2006; Venugopal, 2016).

It has been reported that lipolytic enzymes obtained from guts of Atlantic cod (Gadus morhua) preferentially hydrolyze PUFA over the shorter chain fatty acids which showed a completely opposite fatty acid selectivity to microbial and mammal lipase (Lie and Lambertsen, 1985). Due to their high affinity for long-chain fatty acids, specificity for particular fatty acids and regiospecificity (Diaz-Lopez and Garcia-Carreno, 2000), fish digestive lipases may find applications in the synthesis of structured lipids. Sae-leaw and Benjakul (2018) applied the lipase purified from the liver of seabass to defat fish skin. Defatting with the lipase at 0.30 units $/ g$ dry material for $3 \mathrm{~h}$ at $30{ }^{\circ} \mathrm{C}$ resulted in the removal of $84.57 \%$ fat from the skin. Using lipases, triacylglycerols could be enriched with certain beneficial fatty acids, like eicosapentaenoic acid (EPA), docosahexaenoic acid (DHA), or conjugated linoleic acid (CLA), at a specific position along the glycerol backbone. He and Shahidi (1997) and Wanasundara and Shahidi, (1997) have carried out lipase-assisted hydrolysis of seal blubber oil and menhaden oil in order to enrich their content of $\omega$-3 PUFA in the acylglycerols form. In addition, long-chain PUFAs are highly labile, and lipasecatalyzed lipid modifications could prevent detrimental oxidation and cis-trans isomerization processes (Kurtovic et al., 2009). Marine lipases are likely to have advantages over their microbial or mammalian counterparts because they may operate more efficiently at lower temperatures. Termination of enzymatic reactions could be achieved by smaller changes in temperature as marine lipases often have lower temperature optima and stabilities than lipases from other sources (Rasco and Hultin, 1988). Fish digestive lipases could also be used to carry out lipid interesterification reac- tions (e.g., transesterification) with minimal or no by-products, a problem often encountered in chemical transformations (Kurtovic et al., 2009). Some fish digestive lipases are stereospecific as well as enantiospecific and these features could allow them to be used in the synthesis of specialty pharmaceuticals and agrochemicals, where enantiomerically pure products are desired. Specialty esters for personal care products and environmentally safe surfactants for applications in detergents or as food emulsifiers may also benefit from the use of fish digestive lipases (Kurtovic et al., 2009).

\section{Future considerations}

Living marine resources are limited and shrinking therefore their full utilization is necessary. However, a considerable amount of total catch is discarded as processing leftover. Therefore, better utilization of the catch and processing of discards for the production of novel products is important. This requires addressing challenges related to supplying constant quality by-products. In addition, introduction of the resltant new products to the market also requires special expert involvement. In this, a major difficulty will be the development of economic processes to produce high value products. Sometimes options that might work in one location or area will not be economical in another place. Moreover, what may be appropriate in an industrialized fishery is often not suitable for a small-scale artisanal fishery in developing countries. In order to achieve these goals a concerted effort and overall plan should be in place to sort and handle the by-products onboard and find safe and cost-effective preservation methods and processing for producing high value marketable products that may also improve the health of consumers.

\section{Conclusions}

The seafood industry is originating a large amount of by-products upon processing. These by-products contain valuable protein and lipid fractions as well as a variety of bioactive compounds with potential health benefits. Major ongoing research in recent years have been devised to utilize the ingredients originating from byproducts of the seafood industry in diverse sectors such as food, nutraceutical/functional food, pharmaceutical/biomedical and fine chemicals. Therefore developing new technologies is expected to bring more value to the seafood industry in the future.

\section{References}

Abdel-Aziz, S.M., Kahil, T., and Keera, A.A. (2014). Kinetic behavior of free and in situ immobilized chitosanases produced by the fungus Mucor rouxii. World Appl. Sci. J. 30(1): 01-09.

Abdel-Fattah, W., and Hammad, T. (2001). Chondroitin sulfate and glucosamine: A review of their safety profile. JANA 3: 16-23.

Abdel-Mohsen, A.M., Jancar, J., Massoud, D., Fohlerova, Z., Elhadidy, H., Spotz, Z., and Hebeish, A. (2016). Novel chitin/chitosan-glucan wound dressing: Isolation, characterization, antibacterial activity and wound healing properties. Int. J. Pharm. 510(1): 86-99.

Abdollahi, M., Rezaei, M., Jafarpour, A., and Undeland, I. (2018). Sequential extraction of gel-forming proteins, collagen and collagen hydrolysate from gutted silver carp (Hypophthalmichthys molitrix): a biorefinery approach. Food Chem. 242: 568-578.

Abraha, B., Admassu, H., Mahmud, A., Tsighe, N., Shui, X.W., and Fang, Y. (2018). Effect of processing methods on nutritional and physicochemical composition of fish: A review. MOJ Food Process. Technol. 6(4): 376-382. 
Abugoch, L., Tapia, C., Plasencia, D., Pastor, A., Castro-Mandujano, O., López, L., and Escalona, V.H. (2016). Shelf-life of fresh blueberries coated with quinoa protein/chitosan/sunflower oil edible film. J. Sci. Food Agric. 96(2): 619-626.

Ackman, R.G., Hooper, S.N., and Hingley, J. (1972). The Harbor Seal Phoca vitulina concolor De Kay: Comparative Details of Fatty Acids in Lung and Heart Phospholipids and Triglycerides. Can. J. Biochem. 50: 833-838.

Ackman, R.G., and Ratnayake, W.M.N. (1989). The Role of Fats in Human Nutrition. Academic Press, New York.

Agboh, O.C., and Qin, Y. (1996). Chitin and Chitosan Fibers. Poly. Adv. Technol. 8: 355-365.

Agullo, E., Rodríguez, M.S., Ramos, V., and Albertengo, L. (2003). Present and Future Role of Chitin and Chitosan in Food. Macromol. Biosci. 3: 521-530.

Ahmad, T., Ismail, A., Ahmad, S.A., Khalil, K.A., Kumar, Y., Adeyemi, K.D., and Sazili, A.Q. (2017). Recent advances on the role of process variables affecting gelatin yield and characteristics with special reference to enzymatic extraction: A review. Food hydrocolloids 63: 85-96.

Aiba, S. (1994). Preparation of N-Acetylchitooligosaccharides by Hydrolysis of Chitosan with Chitinase. Carbohyd. Res. 265: 323-328.

Aidos, I., Masbernat-Martinez, S., Luten, J.B., Boom, R.M., and Padt, A.V. (2002). Composition and stability of Herring oil recovered from sorted by-products as compared to oil from mixed by-products. J. Agric. Food Chem. 50: 2818-2824.

Aidos, I., Padt, A.V.D., Boom, R.M., and Luten, J.B. (2003). Quality of crude fish oil extracted from herring byproducts of varying states of freshness. J. Food Sci. 68: 458-465.

Alasalvar, C., Sharhidi, F., and Quantick, P. (2002). Food and health applications of marine nutraceuticals: a review. Seafoods - Quality, Technology and Nutraceutical Applications. pp. 175-204.

Alder-Nissen, J. (1984). Control of the proteolytic reaction and of the level of bitterness in protein hydrolysis processes. J. Chem. Technol. Biotechnol. 34B: 241-246.

Alipour, H.J., Rezaei, M., Shabanpour, B., and Tabarsa, M. (2018). Effects of sulfated polysaccharides from green alga Ulva intestinalis on physicochemical properties and microstructure of silver carp surimi. Food hydrocolloids 74: 87-96.

Alvarez, C., Couso, I., Solas, M.T., and Tejada, M. (1992). Influence of manufacturing process conditions on gels made from sardine surimi. Food Proteins, Structure and Functionality. In: Schwenke, K.D., and Mothes, R. (Ed.). VCH Publishers, Inc., Weinheim, Baden-Württemberg, Germany, pp. 347-353.

Alves, A., Marques, A., Martins, E., Silva, T., and Reis, R. (2017). Cosmetic potential of marine fish skin collagen. Cosmetics 4(4): 39.

Amarowicz, R., and Shahidi, F. (1997). Antioxidant activity of peptide fractions of capelin protein hydrolysates. Food Chem. 58: 355-359.

Ambigaipalan, P., and Shahidi, F. (2017). Bioactive peptides from shrimp shell processing discards: Antioxidant and biological activities. J. Funct. Foods 34: 7-17.

Anderson, L.K. (1975). Extraction of carotenoid pigment from shrimp processing waste. US Patent 3906112.

Appel, L.J., Miller, E.R., Seidler, A.J., and Whelton, P.K. (1993). Does supplementation of diet with 'fish oil' reduce blood pressure? A meta-analysis of controlled clinical trials. Archiv. Inter. Med. 153: 1429-1438.

Arashisar, S., Hisar, O., and Kaban, G. (2009). Effect of chitosan coating on chemical and microbiological properties of Atlantic bonito (Sarda sarda) fillets. J. Food Sci. Technol. 46: 377-379.

Araujo, P., Zhu, H., Breivik, J.F., Hjelle, J.I., and Zeng, Y. (2014). Determination and structural elucidation of triacylglycerols in krill oil by chromatographic techniques. Lipids 49(2): 163-172.

Ariyani, F., and Buckle, K.A. (1991). Ensilaging of prawn heads. ASEAN Food J. 6: 58-63.

Armenta-Lopez, R., Guerrero, I.L., and Huerta, S. (2002). Astaxanthin extraction from shrimp waste by lactic fermentation and enzymatic hydrolysis of the carotenoprotein complex. J. food Sci. 67(3): 10021006.

Arnaud, C., De Lamballerie, M., and Pottier, L. (2018). Effect of high pressure processing on the preservation of frozen and re-thawed sliced cod (Gadus morhua) and salmon (Salmo salar) fillets. High Pressure Res. 38(1): 62-79.
Arnesen, J.A., and Gildberg, A. (2007). Extraction and characterisation of gelatine from Atlantic salmon (Salmo salar) skin. Bioresour. Technol. 98: 53-57.

Arnesen, J.A., and Gildberg, A. (2002). Preparation and Characterisation of Gelatine from the Skin of Harp Seal (Phoca groendlandica). Bioresour. Technol. 82: 191-194.

Arunchalam, K., and Haard, N.F. (1985). Isolation and characterization of pepsin isoenzymes from polar cod (Boreogadus saida). Comp Biochem Physiol. 80B: 467-473.

Aryee, A.N.A., Simpson, B.K., and Villalonga, R. (2007). Lipase fraction from the viscera of grey mullet (Mugil cephalus). Isolation, partial purification, and some biochemical characteristics. Enzyme Microb. Technol. 40: 394-402.

Asgeirsson, B., and Bjarnason, J.B. (1993). Properties of elastase from Atlantic cod, a cold-adapted proteinase. Biochim. et Biophys. Acta. 1164: 91-100.

Asgeirsson, B., Leth-Larsen, R., Thorolfsson, M., Nedertoft, M.M., and Hojrup, P. (1998). The third serine proteinase with chymotrypsin specificity isolated from Atlantic cod (Gadus morhua) is a type-II elastase. Eur. J. Biochem. 255: 638-646.

Aspevik, T., Oterhals, A., Rønning, S.B., Altintzoglou, T., Wubshet, S.G., Gildberg, A., and and Lindberg, D. (2017). Valorization of proteins from co-and by-products from the fish and meat industry. Chemistry and Chemical Technologies in Waste Valorization. Springer, Cham, pp. 123-150.

Atay, E., Fabra, M.J., Martínez-Sanz, M., Gomez-Mascaraque, L.G., Altan, A., and Lopez-Rubio, A. (2018). Development and characterization of chitosan/gelatin electrosprayed microparticles as food grade delivery vehicles for anthocyanin extracts. Food Hydrocolloids 77: 699-710.

Aursand, M., Bleivik, B., Rainuzzo, J.R., Leif, J., and Mohr, V. (1994). Lipid distribution and composition of commercially farmed atlantic salmon (salmosalar). J. Sci. Food Agric. 64(2): 239-248.

Avena-Bustillos, R.J., Olsen, C.W., Olson, D.A., Chiou, B., Yee, E., Bechtel, P.J., and McHugh, T.H. (2006). Water vapour permeability of mammalian and fish gelatine films. J. Food Sci. 71: E202-E207.

Avila Rodríguez, M.I., Rodríguez Barroso, L.G., and Sánchez, M.L. (2018). Collagen: A review on its sources and potential cosmetic applications. J. Cosmet. Dermatol. 17: 20-26.

Azuma, K., Ifuku, S., Osaki, T., Okamoto, Y., and Minami, S. (2014). Preparation and biomedical applications of chitin and chitosan nanofibers. J. Biomed. Nanotech. 10(10): 2891-2920.

Azuma, K., Izumi, R., Osaki, T., Ifuku, S., Morimoto, M., Saimoto, H., Minami, S., and Okamoto, Y. (2015b). Chitin, chitosan, and its derivatives for wound healing: old and new materials. J. Funct. Biomater. 6(1): 104-142.

Azuma, K., Osaki, T., Kurozumi, S., Kiyose, M., Tsuka, T., Murahata, Y., Imagawa, T., Itoh, N., Minami, S., Sato, K., and Okamoto, Y. (2015c). Anti-inflammatory effects of orally administered glucosamine oligomer in an experimental model of inflammatory bowel disease. Carbohydr. Polym. 115: 448-456.

Azuma, K., Osaki, T., Minami, S., and Okamoto, Y. (2015a). Anticancer and anti-inflammatory properties of chitin and chitosan oligosaccharides. J. Funct. Biomater. 6(1): 33-49.

Azuma, K., Osaki, T., Wakuda, T., Tsuka, T., Imagawa, T., Okamoto, Y., and Minami, S. (2012). Suppressive effects of N-acetyl-D-glucosamine on rheumatoid arthritis mouse models. Inflammation 35(4): 1462-1465.

Badii, F., and Howell, N.K. (2006). Fish gelatine: structure, gelling properties and interaction with egg albumen proteins. Food Hydrocolloid. 20: 630-640.

Bae, I., Osatomi, K., Yoshida, A., Osako, K., Yamaguchi, A., and Hara, K. (2008). Biochemical properties of acid-soluble collagens extracted from the skins of underutilised fishes. Food Chem. 108: 49-54.

Balian, G., and Bowes, J. (1977). The Structure and Properties of Collagen. The Science and Technology of Gelatine. In: Ward, A.G., and Courts, A. (Ed.). Academic Press, London, pp. 1-30.

Balkenende, D.W., Winkler, S.M., and Messersmith, P.B. (2019). MarineInspired Polymers in Medical Adhesion. Eur. Polym. J. 116: 134-143.

Balti, R., Barkia, A., Bougatef, A., Ktari, N., and Nasri, M. (2009). A heatstable trypsin from the hepatopancreas of the cuttlefish (Sepia officinalis): Purification and characterisation. Food Chem. 113(1): 146-154. 
Bang, H.O., Dyerberg, J., and Hjorne, N. (1976). The composition of food consumed by Greenland Eskimos. Acta. Med. Scand. 200: 59-73.

Baranowski, E.S., Nip, W.K., and Moy, J.H. (1984). Partial characterization of a crude enzyme extract from the freshwater prawn, Macrobrachium rosenbergii. J. Food Sci. 49: 1494-1495.

Barlow, S., and Young, V. (1988). New uses for fish oils. Food Manuf. 63: 75.

Barratt, A., and Montano, R. (1986). Shrimp heads-a new source of protein. INFOFISH Marketing Digest 4(86): 21-22.

Barriada, J.L., Herrero, R., Prada-Rodríguez, D., and de Vicente, M.E.S (2007). Waste spider crab shell and derived chitin as low-cost materials for cadmium and lead removal. J. Chem. Technol. Biotechnol. 82 39-46.

Batista, I. (2007). By-catch, underutilized species and underutilized fish parts as food ingredients. Maximizing the value of marine by-products. In: Shahidi, F. (Ed.). CRC Press, Boca Raton, FL, pp. 171-195.

Baxter, S.R., and Skonberg, D.I. (2008). Gelation properties of previously cooked minced meat from Jonah crab (Cancer borealis) as affected by washing treatment and salt concentration. Food Chem. 109: 332-9.

Baxter, S.R., and Skonberg, D.I. (2006). Thermal gelation of previously cooked minced meat from Jonah crab (Cancer borealis). J. Food Sci. 71: C499-503.

Bechtel, P.J. (2007). By-products from seafood processing for aquaculture and animal feeds. Maximizing the value of marine by-products. In: Shahidi, F. (Ed.). CRC Press, Boca Raton, FL, pp. 435-449.

Beindorff, C.M., and Zuidam, N.J. (2010). Microencapsulation of fish oil. Encapsulation technologies for active food ingredients and food processing. Springer, New York, NY, pp. 161-185.

Belluzzi, A., Brignola, C., Campieri, M., Pera, A., Boschi, S., and Miglioli, M. (1996). Effect of an enteric-coated fish-oil preparation on relapses in Crohn's disease. N. Engl. J. Med. 334: 1557-1560.

Belouafa, S., Bourja, L., Villain, S., Tayane, S., Bennamara, A., and Abourriche, A. (2018). Biocomposite Based on Collagen/Calcium Salts Extraction from Sardine Scales. Smart Application and Data Analysis for Smart Cities (SADASC'18). Available at SSRN: https://ssrn.com/ abstract $=3186344$.

Benavente, M., Moreno, L., and Martinez, J. (2011). Sorption of heavy metals from gold mining wastewater using chitosan. J. Taiwan Inst. Chem. Eng. 42(6): 976-988.

Bendich, A., and Olson, J.A. (1989). Biological actions of carotenoids. Fed. Am. Soc. Exp. Biochem. 3: 1927-1932.

Beppu, F., Yasuda, K., Okada, A., Hirosaki, Y., Okazaki, M., and Gotoh, N. (2017). Comparison of the distribution of unsaturated fatty acids at the sn-2 position of phospholipids and triacylglycerols in marine fishes and mammals. J. Oleo Sci. 66(11): 1217-1227.

Bernhardt, A., Paul, B., and Gelinsky, M. (2018). Biphasic scaffolds from marine collagens for regeneration of osteochondral defects. Mar. Drugs 16(3): 91.

Berrocal, R., Chanton, S., Juillerat, M.A., Pavillard, B., Scherz, J.C., and Jost, R. (1989). Tryptic phosphopeptides from whole casein. II. Physiochemical properties related to the solubilization of calcium. J. Dairy. Res. 56: 335-341.

Best, S.M., Porter, A.E., Thian, E.S., and Huang, J. (2008). Bioceramics: past, present and for the future. J. Eur. Ceram. Soc. 28(7): 1319-1327.

Biesiot, P.M., and Capuzzo, J.M. (1990). Digestive protease, lipase, and amylase activities in stage I larvae of the American lobster, Homarus americanus. Comp. Biochem. Physiol. A: Comp. Physiol. 95: 47-54.

Bilal, M., Iqbal, H.M., Hu, H., Wang, W., and Zhang, X. (2017). Enhanced bio-catalytic performance and dye degradation potential of chitosanencapsulated horseradish peroxidase in a packed bed reactor system. Sci. Total Environ. 575: 1352-1360.

Bimbo, A.P. (2009). Raw material sources for the long-chain omega-3 market: Trends and sustainability. Part 2. 99th AOCS Annual Meeting and Expo in Seattle.

Binsi, P.K., and Shamasundar, B.A. (2012). Purification and characterisation of transglutaminase from four fish species: Effect of added transglutaminase on the viscoelastic behaviour of fish mince. Food Chem. 132(4): 1922-1929.

Bjerkeng, B., Storebakken, T., and Liaaen-Jensen, S. (1992). Pigmentation of rainbow trout from start feeding to sexual maturation. Aquacul. 108: 333-346.

Blanco, M., Fraguas, J., Sotelo, C., Pérez-Martín, R., and Vázquez, J. (2015).
Production of chondroitin sulphate from head, skeleton and fins of Scyliorhinus canicula by-products by combination of enzymatic chemical precipitation and ultrafiltration methodologies. Mar. Drugs 13(6): 3287-3308.

Blanco, M., Vázquez, J., Pérez-Martín, R., and Sotelo, C. (2017). Hydrolysates of fish skin collagen: an opportunity for valorizing fish industry byproducts. Mar. Drugs 15(5): 131.

Blumer, M. (1967). Hydrocarbons in digestive tract and liver of a basking shark. Sci. 156: 390-391.

Bokura, H., and Kobayashi, S. (2003). Chitosan decreases total cholesterol in women: a randomized, double-blind, placebo-controlled trial. Eur. J. Clin. Nutr. 57: 721-725.

Boran, G., and Regenstein, J.M. (2009). Optimization of gelatin extraction from silver carp skin. J. Food Sci. 74(8): E432-E441.

Borderías, A.J., Sánchez-Alonso, I., and Pérez-Mateos, M. (2005). New applications of fibres in foods: Addition to fishery products. Trends Food Sci. Technol. 16: 458-465.

Boskou, D. (1998). Frying temperatures and minor constituents of oils and fats. Grasas y aceites 49(3-4): 326-330.

Boskou, D. (2008). Olive oil: minor constituents and health. CRC press.

Bougatefa, A., Arroumeb, N., Mannia, L., Ravallecb, R., Barkiaa, A., Guillochonb, D., and Nasri, M. (2010). Purification and identification of novel antioxidant peptides from enzymatic hydrolysates of sardinelle (Sardinella aurita) by-products proteins. Food Chem. 118: 559-565.

Bourtoom, T. (2008). Edible films and coatings: characteristics and properties. Int. Food Res. J. 15: 237-248.

Braun, J., Chanu, P.L., and Goffic, F.L. (1989). The Immobilization of Penicillin G Acylase on Chitosan. Biotechnol. Bioeng. 33: 242-246.

Brennan, R.A., Sanford, R.A., and Werth, C.J. (2006b). Biodegradation of Tetrachloroethene by Chitin Fermentation Products in a Continuous Flow Column System. J. Environ. Eng. 132: 664-673.

Brennan, R.A., Sanford, R.A., and Werth, C.J. (2006a). Chitin and corncobs as electron donor sources for the reductive dechlorination of tetrachloroethene. Water Res. 40: 2125-2134.

Britton, G. (1985). General carotenoid methods. Methods in Enzymology. In: Law, J.H., and Rilling, H.C. (Ed.). Academic Press, New York, pp. 113-149.

Broadwater, M.H., Seaborn, G.T., and Schwacke, J.H. (2013). Forensic identification of seal oils using lipid profiles and statistical models. J. Forensic Sci. 58(2): 336-343.

Broughton, K.S., Johnson, C.S., Pace, B.K., Liebman, M., and Kleppinger, K.M. (1997). Reduced asthma symptoms with $n-3$ fatty acid ingestion are related to 5-series leukotriene production. Am. J. Clin. Nutr. 65: 1011-1017.

Brunborg, L.A., Julshamn, K., Nortvedt, R., and Frøyland, L. (2006). Nutritional composition of blubber and meat of hooded seal (Cystophora cristata) and harp seal (Phagophilus groenlandicus) from Greenland. Food Chem. 96: 524-531.

Buchecker, R.A. (1982). Chemist's view of animal carotenoids. Carotenoid Chemistry and Biochemistry. In: Britton, G., and Goodwin, T.W. (Ed.) Pergamon Press, Oxford, pp. 175-193.

Bucsi, L., and Poor, G. (1998). Efficacy and tolerability of oral chondroitin sulfate as a symptomatic slow-acting drug for osteoarthritis (SYSADOA) in the treatment of knee osteoarthritis. Osteoarthr. Cartilage. 6: 31-36.

Budavari, S. (1996). Merck Index. Merck and Co., Whitehouse Station, NJ.

Burke, J.M., Staples, C.R., Risco, C.A., de LaSota, R.L., and Thatcher, W.W. (1997). Effect of ruminant grade Menhaden fish meal on reproductive and productive performance of lactating dairy cows. J. Dairy Sci. 80: 3386-3398.

Butler, B.L., Vergaro, P.J., Testin, R.F., Bunn, J.M., and Wiles, J.L. (1996). Mechanical and barrier properties of edible chitosan films as affected by composition and storage. J. Food Sci. 61: 953-961.

Byun, S.M., No, H.K., Hong, J.H., Lee, S.I., and Prinyawiwatkul, W. (2013). Comparison of physicochemical, binding, antioxidant and antibacterial properties of chitosans prepared from ground and entire crab leg shells. Int. J. Food Sci. Technol. 48(1): 136-142.

Cai, L., Feng, J., Regenstein, J., Lv, Y., and Li, J. (2017). Confectionery gels: Effects of low calorie sweeteners on the rheological properties and microstructure of fish gelatin. Food hydrocolloids 67: 157-165.

Calder, P.C. (2018). Very long-chain n-3 fatty acids and human health: fact, 
fiction and the future. Proc. Nutr. Soc. 77(1): 52-72.

Calder, P.C. (2014). Very long chain omega-3 (n-3) fatty acids and human health. Eur. J. Lipid Sci. Technol. 116(10): 1280-1300.

Calder, P.C., Yaqoob, P., Thies, F., Wallace, F.A., and Miles, E.A. (2002). Fatty acids and lymphocyte functions. Br. J. Nutr. 87: S31-S48.

Campoy, C., Escolano-Margarit, M.V., Anjos, T., Szajewska, H., and Uauy, R. (2012). Omega 3 fatty acids on child growth, visual acuity and neurodevelopment. Br. J. Nutr. 107(S2): S85-S106.

Caner, C. (2005). The effect of edible eggshell coatings: on egg quality and consumer perception. J. Sci. Food Agric. 85: 1897-1902.

Caner, C. (2005). The effect of edible eggshell coatings: on egg quality and consumer perception. J. Sci. Food Agric. 85: 1897-1902.

Cao, S.M., Wu, Y.Y., Li, L.H., Yang, X.Q., Chen, S.J., Hu, X., and Ma, H.X. (2018). Activities of Endogenous Lipase and Lipolysis Oxidation of Low-Salt Lactic Acid-Fermented Fish (Decapterus maruadsi). J. Oleo Sci. 67(4): 445-453.

Cappannella, E., Benucci, I., Lombardelli, C., Liburdi, K., Bavaro, T., and Esti, M. (2016). Immobilized lysozyme for the continuous lysis of lactic bacteria in wine: Bench-scale fluidized-bed reactor study. Food Chem. 210: 49-55.

Carney, S.L., and Muir, H. (1988). The structure and function of cartilage proteoglycans. Physiol. Rev. 68: 858-910.

Carvalho, A., Marques, A., Silva, T., and Reis, R. (2018). Evaluation of the Potential of Collagen from Codfish Skin as a Biomaterial for Biomedical Applications. Marine drugs 16(12): 495.

Castillo, R., Negre-Sadargeus, G., and Lenel, R. (1982). General survey of the carotenoids in crustacean. Carotenoid Chemistry and Biochemistry. In: Britton, G., and Goodwin, T.W. (Ed.). Pergamon Press, Oxford, p. 211.

Centenaro, G.S., Salas-Mellado, M., Pires, C., Batista, I., Nunes, M.L., and Prentice, C. (2014). Fractionation of protein hydrolysates of fish and chicken using membrane ultrafiltration: investigation of antioxidant activity. Appl. Biochem. Biotechnol. 172(6): 2877-2893.

Chalamaiah, M., Hemalatha, R., and Jyothirmayi, T. (2012). Fish protein hydrolysates: proximate composition, amino acid composition, antioxidant activities and applications: a review. Food Chem. 135(4): 3020-3038.

Chan, K.O., Tong, H.H., and Ng, G.Y. (2016). Topical fish oil application coupling with therapeutic ultrasound improves tendon healing. Ultrasound Med. Biol. 42(12): 2983-2989.

Chandra, M.V., and Shamasundar, B.A. (2015). Texture profile analysis and functional properties of gelatin from the skin of three species of fresh water fish. Int. J. Food Prop. 18(3): 572-584.

Chantachum, S., Benjakul, S., and Sriwirat, N. (2000). Separation and quality of fish oil from precooked and non-precooked tuna heads. Food Chem. 69: 289-294.

Charest, D.J., Bulaban, M.O., Marshall, M.R., and Cornell, J.A. (2001). Astaxanthin extraction from crawfish shells by supercritical $\mathrm{CO}_{2}$ with ethanol as cosolvent. J. Aqua Food Prod. Technol. 10: 79-93.

Charoenvuttitham, P., Shi, J., and Mittal, G.S. (2006). Chitin extraction from black tiger shrimp (Penaeus monodon) waste using organic acids. Sep. Sci. Technol. 41(06): 1135-1153.

Chatelet, C., Damour, O., and Domard, A. (2001). Influence of the degree of acetylation on some biological properties of chitosan films. Biomaterials 22(3): 261-268.

Cheba, B.A. (2011). Chitin and chitosan: marine biopolymers with unique properties and versatile applications. Global Journal Biotechnol., Biochem. 6(3): 149-153.

Chen, C.S., Liau, W.Y., and Tsai, G.J. (1998). Antibacterial effects of Nsulfobenzoyl chitosan and its application to oyster preservation. J. Food Prot. 61: 1124-1128.

Chen, H.M., and Meyers, S.P. (1982). Extraction of astaxanthin pigment from crawfish waste using a soy oil process. J. Food Sci. 47: 892-896.

Chen, H.M., and Meyers, S.P. (1982). Extraction of astaxanthin pigment from crawfish waste using a soy oil process. J. Food Sci. 47: 892-896.

Chen, X., Wu, J., Chen, Y., and Liu, Z. (2016). Research progress of highvalue utilization of pelagic fishes and by-products. J. Food Saf. Qual. 7(3): 1226-1232.

Cheow, C.S., Norizah, M.S., Kyaw and, Z.Y., and Howell, N.K. Preparation and characterisation of gelatines from the skins of sin croaker (Johnius dussumieri) and shortfin scad (Decapterus macrosoma). Food
Chem. 101: 386-391.

Chesley, L.C. (1934). The concentrations of proteases, amylase, and lipase in certain marine fishes. Biol. Bull. 66: 133-144.

Cheung, I.W.Y., and Li-Chan, E.C.Y. (2010). Angiotensin-I-converting enzyme inhibitory activity and bitterness of enzymatically-produced hydrolysates of shrimp (Pandalopsis dispar) processing byproducts investigated by Taguchi design. Food Chem. 122: 1003-1012.

Chi, C.F., Wang, B., Wang, Y.M., Zhang, B., and Deng, S.G. (2015). Isolation and characterization of three antioxidant peptides from protein hydrolysate of bluefin leatherjacket (Navodon septentrionalis) heads. J. Funct. Foods 12: 1-10.

Cho, S.H., Jahncke, M.L., Chin, K.B., and Eun, J.B. (2006). The effect of processing conditions on the properties of gelatine from skate (Raja kenojei) skins. Food Hydrocolloid. 20: 810-816.

Cho, S.M., Gu, Y.S., and Kim, S.B. (2005). Extracting optimization and physical properties of yellowfin tuna (Thunnus albacares) skin gelatine compared to mammalian gelatines. Food Hydrocolloid. 19: 221-229.

Choi, J.S., Lee, C.K., Jeon, Y.J., Byun, H.G., and Kim, S.K. (1999). Properties of the ceramic composites and glass ceramics prepared by using the natural hydroxyapatite derived from tuna bone. J. Korean Indus. Eng. Chem. 10: 394-399.

Choi, S.-S., and Regenstein, J.M. (2000). Physicochemical and sensory characteristics of fish gelatine. J. Food Sci. 65: 194-199.

Choi, S.S., and Regenstein, J.M. (2000). Physicochemical and sensory characteristics of fish gelatine. J. Food Sci. 65: 194-199.

Choubert, G., Gomez, R., and Milicua, J.C.G. (1994). Response of serum carotenoid level to dietary astaxanthin and canthaxanthin in immature rainbow trout, oncorhynchus mykiss, Comp. Biochem. Physiol. 109A: 1001-1006.

Choudhury, G.S., and Bublitz, C.G. (1996). Computer-based controls in fish processing industry. Computerized control systems in the food industry. In: Mittal, G.S. (Ed.). Marcel Dekker Inc., New York, pp. 513-538.

Christensen, J.H., Korup, E., Aaroe, J., Toft, E., Moller, J., Rasmussen, K. and et al (1997). Fish consumption, n-3 fatty acids in cell membranes, and heart rate variability in survivors of myocardial infraction with left ventricular dysfunction. Am. J. Cardiol. 79: 1670-1673.

Chung, Y.C., Su, Y.P., Chen, C.C., Jia, G., Wang, H.L., Wu, J.C.G., and Lin, J.G. (2004). Relationship between antibacterial activity of chitosan and surface characteristics of cell wall. Acta Pharmacol. Sinica 25 932-936.

Clark, J., MacDonald, N.L., and Stark, J.R. (1985). Metabolism in marine flatfish. II. Protein digestion in Dover sole (Solea solea L.). Comp. Biochem. Physiol. B Biochem. Mol. Biol. 81(1): 217-222.

Clark, J., Quayle, K.A., Macdonald, N.L., and Stark, J.R. (1988). Metabolism in marine flatfish-V. chitinolytic activities in dover sole, Solea solea (L.). Comp. Biochem. Physiol. 90B: 379-384.

Cohen, E. (2001). Chitin synthesis and inhibition: a revisit. Pest Mgt. Sci. 57: $946-950$

Cohen, T., and Gertler, A. (1981). Pancreatic proteolytic enzymes from carp Cyprinus carpio I. Purification and physical properties of trypsin, chymosin, elastase and carboxypeptidase B. Comp. Biochem. Physiol. 69B: 647-653.

Coletta, J.M., Bell, S.J., and Roman, A.S. (2010). Omega-3 fatty acids and pregnancy. Rev. Obstet. Gynecol. 3(4): 163.

Coma, V. (2008). Bioactive Chitosan-based Substances and Films. Current Research and Development on Chitin and Chitosan in Biomaterials. In: Jayakumar, R., and Prabaharan, M. (Ed.). Research Signpost, Trivandrum, Kerala, India, pp. 21-51.

Coma, V., Deschamps, A., and Martial-Gros, A. (2003). Bioactive Packaging Materials from Edible Chitosan Polymer-Antimicrobial Activity Assessment on Dairy-Related Contaminants. J. Food Sci. 68: 27882792.

Conway, J.R.W., Vennin, C., Cazet, A.S., Herrmann, D., Murphy, K.J., Warren, S.C., Wullkopf, L., Boulghourjian, A., Zaratzian, A., Da Silva, A.M., and Pajic, M. (2017). Three-dimensional organotypic matrices from alternative collagen sources as pre-clinical models for cell biology. Sci. Rep. 7: 16887.

Cremades, O., Ponce, E., Corpas, R., Gutiérrez, J.F., Jover, M., Alvarez-Ossorio, M.C., Parrado, J., and Bautista, J. (2001). Processing of crawfish (Procambarus clarkii) for the preparation of carotenoproteins and chitin. J. Agri. Food Chem. 49: 5468-5472. 
Cudennec, B., Ravallec-Plé, R., Courois, E., and Fouchereau-Peron, M. (2008). Peptides from fish and crustacean by-products hydrolysates stimulate cholecystokinin release in STC-1 cells. Food Chem. 111: 4970-975.

Cuero, R.G., Osuji, G., and Washington, A. (1991). N-Carboxymethyl chitosan inhibition of aflatoxin production: role of zinc. Biotechnol. Lett. 13: 441-444.

Darmadji, P., and Izumimoto, M. (1994). Effect of chitosan in meat preservation. Meat Sci. 38: 243-254.

Davies, D.H., Elson, C.M., and Hayes, E.R. (1989). N, O-carboxymethyl chitosan, a new water soluble chitin derivative. Chitin and Chitosan Source, Chemistry, Biochemistry, Physical Properties, and Application. In: Skjak-Braek, G., Anthosen, T., and Sandford, P. (Ed.). Elsevier Applied Science, New York, pp. 467-472.

Davis, R.W. (2014). A review of the multi-level adaptations for maximizing aerobic dive duration in marine mammals: from biochemistry to behavior. J. Comp. Physiol. B 184(1): 23-53.

De-Vecchi, S.D., and Coppes, Z. (1996). Marine fish digestive proteasesrelevance to food industry and south-west Atlantic region-a review. J. Food Biochem. 20: 193-214.

De La Fuente-Betancourt, G., Garcla-Carreno, F., Navarrete Del Toro, M.A., Cordova-Murueta, J.H., and Lugo-Sanchez, M.E. (2009). Protein solubility and production of gels from jumbo squid. J. Food Biochem. 33 273-90.

Deepthi, S., Venkatesan, J., Kim, S.K., Bumgardner, J.D., and Jayakumar, R. (2016). An overview of chitin or chitosan/nano ceramic composite scaffolds for bone tissue engineering. Int. J. Biol. Macromol. 93 1338-1353.

Deeslie, W.D., and Cheryan, M. (1991). Fractionation of soy protein hydrolysates using ultrafiltration membranes. J. Food Sci. 57: 411-413.

Dehghani, S., Hosseini, S.V., and Regenstein, J.M. (2018). Edible films and coatings in seafood preservation: A review. Food Chem. 240: 505513.

Del Giudice, G., Fragapane, E., Bugarini, R., Hora, M., Henriksson, T., Palla E., O'Hagan, D., Donnelly, J., Rappuoli, R., and Podda, A. (2006). Vaccines with the MF59 adjuvant do not stimulate antibody responses against squalene. Clin. Vaccine Immunol. 13(9): 1010-1013.

Demetriou, M., Brandt, A., Friedmann, P., and Dennis, J. (2018). U.S. Patent Application No. 15/547,496.

Deprez, P.P., Volkman, J.K., and Davenport, S.R. (1990). Squalene Content and Neutral Lipid Composition of Livers from Deep-sea Sharks. Caught in Tasmanian Waters. Aust. J. Mar. Freshwater Res. 41: 37587.

Devlieghere, F., Vermeulen, A., and Debevere, J. (2004). Chitosan: antimicrobial activity, interactions with food components and applicability as a coating on fruit and vegetables. Food Microbiol. 21: 703-714.

Di Pasquale, M. (1997). Dietary protein and amino acids. Amino Acids and Proteins for the Athlete: The Anabolic Edge. CRC Press, Boca Raton, FL, p. 63.

Diaz-Lopez, M., and Garcia-Carreno, F. (2000). Applications of fish and shellfish enzymes in food and feed products. Seafood Enzymes. Utilization and Influence on Postharvest Seafood Quality. In: Haard, N.F. and Simpson, B.K. (Ed.). Marcel Dekker, New York, pp. 571-618.

Djabourov, M., Lechaire, J.P., and Gaill, F. (1993). Structure and rheology of gelatine and collagen gels. Biorheol. 30: 191-205.

Djellouli, M., López-Caballero, M.E., Arancibia, M.Y., Karam, N., and Martínez-Alvarez, O. (2019). Antioxidant and Antimicrobial Enhancement by Reaction of Protein Hydrolysates Derived from Shrimp By-Products with Glucosamine. Waste Biomass Valorization. pp. 1-15.

Doğaç, Y.I., and Teke, M. (2016). Synthesis and characterisation of biocompatible polymer-conjugated magnetic beads for enhancement stability of urease. Appl. Biochem. Biotechnol. 179(1): 94-110.

Dorman, T., Bernard, L., Glaze, P., Hogan, J., Skinner, R., Nelson, D., Bowker, L., and Head, D. (1995). The effectiveness of Garum armoricum (stabilium) in reducing anxiety in college students. J. Adv. Med. 8 193-200.

Dutta, P.K., Dutta, J., and Tripathi, V.S. (2004). Chitin and chitosan: Chemistry, properties and applications. .

Dutta, P.K., Tripath, S., Mehrotra, G.K., and Dutta, J. (2009). Perspectives for chitosan based antimicrobial films in food applications. J. Food Chem. 114: 1173-1182.

Eastoe, J.E., and Leach, A.A. (1977). Chemical constitution of gelatine. The science and technology of gelatine. In: Ward, A.G., and Courts, A (Ed.). Academic Press, London, pp. 73-107.

Ehrlich, H., Wysokowski, M., Żółtowska-Aksamitowska, S., Petrenko, I., and Jesionowski, T. (2018). Collagens of poriferan origin. Mar. Drugs 16(3): 79.

El Ghaouth, A., Arul, J., Grenier, J., and Asselin, A. (1992). Antifungal activity of chitosan on two postharvest pathogens of strawberry fruits. Phytopathol. 82: 398-402.

Elsen, A., and Jeffrey, J.J. (1969). An extractable collagenase from crustacean hepatopancreas. Biochim. et Biophys. 191: 517-526.

Elson, C.M., Hayes, E.R., and Lidster, P.D. (1985). Development of the differentially permeable fruit coating Nutri-Save ${ }^{\circledR}$ for the modified atmosphere storage of fruit. Proceedings of the 4th National Controlled Atmosphere Research Conference: Controlled Atmosphere for Storage and Transport of Perishable Agricultural Commodities. pp. 248-262.

Engelhardt, F.R., and Walker, B.L. (1974). Fatty acid composition of the harp seal,Pagophilus groenlandicus (Phoca groenlandica). Comp. Biochem. Physiol. 47B: 169-179.

Ennaas, N., Hammami, R., Gomaa, A., Bédard, F., Biron, É., Subirade, M. and and Fliss, I. (2016). Collagencin, an antibacterial peptide from fish collagen: Activity, structure and interaction dynamics with membrane. Biochem. Biophys. Res. Commun. 473(2): 642-647.

Falch, E., Sandbakk, M., and Aursand, M. (2007). On-board handling of marine by-products to prevent microbial spoilage, enzymatic reactions and lipid oxidation. Maximizing the value of marine by-products. In: Shahidi, F. (Ed.). CRC Press, Boca Raton, FL, pp. 47-64.

Fang, S.W., Li, C.F., and Shih, D.Y.C. (1994). Antifungal activity of chitosan and its preservative effect on low sugar candied kumquat. J. Food Prot. 57: 136-140.

FAO. (2008). http://www.fao.org/DOCREP/003/X9199E/X9199E04.htm.

FAO. (2012). http://www.fao.org/docrep/016/i2727e/i2727e.pdf.

FAO. (2012). World Review of Fisheries and Aquaculture, http://www.fao. org/3/i2727e/i2727e01.pdf.

FAO. (2018a). The State of World Fisheries and Aquaculture, http://www. fao.org/3/19540EN/i9540en.pdf.

FAO. (2018b). GLOBEFISH - Information and Analysis on World Fish Trade http://www.fao.org/in-action/globefish/market-reports/resourcedetail/en/c/1181786/.

FAO. (2018c). GLOBEFISH - Information and Analysis on World Fish Trade http://www.fao.org/in-action/globefish/market-reports/resourcedetail/en/c/1181789/.

Felberg, H.S., Hagen, L., Slupphaug, G., Batista, I., Nunes, M.L., Olsen, R.L., and Martinez, I. (2010). Partial characterisation of gelatinolytic ac tivities in herring (Clupea harengus) and sardine (Sardina pilchardus) possibly involved in post-mortem autolysis of ventral muscle. Food Chem. 119(2): 675-683.

Felse, P.A., and Panda, T. (1999). Studies on Application of Chitin and its Derivatives. Bioprocess Eng. 20: 505-512.

Fernández-Díaz, M.D., Montero, P., and Gómez-Guillén, M.C. (2003). Effect of freezing fish skins on molecular and rheological properties of extracted gelatine. Food Hydrocolloid. 17: 281-286.

Fernández-Díaz, M.D., Montero, P., and Gomez-Guillen, M.C. (2001). Gel properties of collagens from skins of cod (Gadus morhua) and hake (Merluccius merluccius) and their modification by the coenhancers magnesium sulphate, glycerol and transglutaminase. Food Chem. 74: 161-167.

Fernandez-Saiz, P., Lagaron, J.M., and Ocio, M.J. (2009). Optimization of the biocide properties of chitosan for its application in the design of active films of interest in the food area. Food Hydrocolloid. 23: 913-921.

Ferraro, V., Cruz, I.B., Jorge, R.F., Malcata, F.X., Pintado, M.E., and Castro, P.M. (2010). Valorisation of natural extracts from marine source focused on marine by-products: A review. Food Res. Int. 43(9): 22212233

Food and Drug Regulation. (2005). Available from: http://laws.justice. gc.ca/en/F-27/C.R.C.-c.870/124366.html\#rid-12437, Department of justice, Canada, 2005.

Foroutani, M.B., Parrish, C.C., Wells, J., Taylor, R.G., Rise, M.L., and Shahidi, F. (2018). Minimizing marine ingredients in diets of farmed Atlantic salmon (Salmo salar): Effects on growth performance and muscle li- 
pid and fatty acid composition. PloS one 13(9): e0198538.

Fouchereau-Peron, M., Duvail, L., Michel, C., Gildberg, A., Batista, I., and Gal, Y.I. (1999). Isolation of an acid fraction from a fish protein hydrolysate with a calcitonin-gene-related-peptide-like biological activity. Biotechnol. Appl. Biochem. 29: 87-92.

Friedman, S.J., and Skehan, P. (1980). Membrane-active drugs potentiate the killing of tumor cells by D-glucosamine. Proc Natl Acad Sci U S A. 77: 1172-1176.

Fritsche, K.L., Cassity, N.A., and Huang, S. (1991). Effect of dietary fat source on antibody production and lymphocyte proliferation in chickens. Poult. Sci. 70: 611-617.

Fuke, S., Watanabe, K., Sakai, H., and Konosu, S. (1989). Extractive Components of Dried Skipjack. Nippon Shokuhin Kogyo Gakkaishi. 36(1): 67-70.

Gómez-Guillén, M.C., Giménez, B., and Montero, P. (2005). Extraction of gelatin from fish skins by high pressure treatment. Food hydrocolloids 19(5): 923-928.

Gómez-Guillén, M.C., Turnay, J., Fernández-Díaz, M.D., Ulmo, N., Lizarbe, M.A., and Montero, P. (2002). Structural and physical properties of gelatine extracted from different marine species: a comparative study. Food Hydrocolloid. 16: 25-34.

Galal-Khallaf, A., Ardura, A., Borrell, Y.J., and Garcia-Vazquez, E. (2016). Towards more sustainable surimi? PCR-cloning approach for DNA barcoding reveals the use of species of low trophic level and aquaculture in Asian surimi. Food control 61: 62-69.

Galanakis, C.M. (2013). Emerging technologies for the production of nutraceuticals from agricultural by-products: a viewpoint of opportunities and challenges. Food Bioprod. Process. 91(4): 575-579.

Galetti, J.A. (2010). Mechanical processing of European Green Crab (Carcinus maenas): the development of a value-added product and the use of restructuring additives to increase the functional properties of green crab patties. Electronic Theses and Dissertations. .

Gao, Y., Fukushima, H., Deng, S., Jia, R., Osako, K., and Okazaki, E. (2018). Effect of emulsifying stability of myofibrillar protein on the gel properties of emulsified surimi gel. Food Sci. Nutr. 6(5): 1229-1237.

Garbisu, C., and Alkorta, I. (2001). Phytoextraction: A cost effective plantbased technology for the removal of metals from the environment. Biores. Technol. 77: 229-236.

Garcia-Carreno, F.L., Hernandez-Cortes, M.P., and Haard, N.F. (1994). Enzymes with peptidase and proteinase activity from the digestive system of a freshwater and marine decapod. J. Agri. Food Chem. 42: 1456-1461.

Garner, S.C., Anderson, J.J.B., and Ambrose, W.W. (1996). Skeletal tissues and mineralization. Calcium and phosphorous in health and disease. In: Anderson, J.J.B., and Garner, S.C. (Ed.). CRC Press, New York, pp. 97-117.

Garnett, M.C. (1999). Gene-delivery systems using cationic polymers. Crit. Rev. Ther. Drug Carrier Syst. 16: 147-207.

Garnjanagoonchorn, W., Wongekalak, L., and Engkagul, A. (2007). Determination of chondroitin sulfate from different sources of cartilage. Chem. Eng. Process. 46: 65-471.

Gatti, A.M., Zaffe, D., and Poli, G.P. (1990). Behaviour of tricalcium phosphate and hydroxyapatite granules in sheep bone defects. Biomater. 11: 513-17.

Gelman, A., Mokadi, S., and Cogan, U. (1989). The thermal properties of intestinal alkaline phosphatase of three kinds of deep-water fish. Comp Biochem Physiol. 94B: 113-116.

Georgantelis, D., Blekas, G., Katikou, P., Ambrosiadis, I., and Fletouris, D.J. (2007). Effect of rosemary extract, chitosan and $\alpha$-tocopherol on lipid oxidation and color stability during frozen storage of beef burgers. Meat Sci. 75(2): 256-264.

Gerasimova, N.A., and Kupina, N.M. (1996). Properties of proteases from internal organs of the king crab. Appl. Biochem. Microbiol. 32: 375378.

Gerente, C., Lee;, V.K.C., Le Cloirec, P., and McKay, G. (2007). Application of Chitosan for the Removal of Metals from Wastewaters by Adsorption: Mechanisms and Models Review. Crit. Rev. Environ. Sci. Technol. 37: 41-127.

Gevaert, B., Veryser, L., Verbeke, F., Wynendaele, E., and De Spiegeleer, B. (2016). Fish hydrolysates: A regulatory perspective of bioactive peptides. Protein Peptide Let. 23(12): 1052-1060.
Ghanbari, M., Jami, M., Domig, K.J., and Kneifel, W. (2013). Seafood biopreservation by lactic acid bacteria-a review. LWT-Food Sci. Technol. 54(2): 315-324.

Ghaouth, A.E., Arul, J., Ponnampalam, R., and Boulet, M. (1991a). Chitosan coating effect on stability of fresh strawberries. J. Food Sci. 57: 1618-1620.

Ghaouth, A.E., Arul, J., Ponnampalam, R., and Boulet, M. (1991b). Use of chitosan coating to reduce water loss and maintain quality of cucumber and bell pepper fruits. J. Food Process. Preserv. 15: 359-368.

Ghidalia, W. (1985). Structural and biological aspects of pigments. The biology of crustacea. In: Bliss, D.E., and Mantel, L.H. (Ed.). Academic Press, New York, p. 301.

Ghorbanzade, T., Jafari, S.M., Akhavan, S., and Hadavi, R. (2017). Nanoencapsulation of fish oil in nano-liposomes and its application in fortification of yogurt. Food Chem. 216: 146-152.

Gigliotti, J.C., Davenport, M.P., Beamer, S.K., Tou, J.C., and Jaczynski, J. (2011). Extraction and characterisation of lipids from Antarctic kril (Euphausia superba). Food Chem. 125(3): 1028-1036.

Gilani, S.L., Najafpour, G.D., Moghadamnia, A., and Kamaruddin, A.H. (2016). Stability of immobilized porcine pancreas lipase on mes oporous chitosan beads: a comparative study. J. Mol. Catal. B. Enzym. 133: 144-153.

Gildberg, A. (1988). Aspartic proteinases in fishes and aquatic invertebrates. Comp. Biochem. Physiol. 91B: 425-435.

Gildberg, A. (1992). Recovery of proteinases and protein hydrolysates from fish viscera. Bioresource Technol. 39: 271-276.

Gildberg, A. (2002). Enhancing returns from greater utilization. Safety and Quality Issues in Fish Processing. In: Bremner, H.A. (Ed.). Woodhead Publishing Ltd., Cambridge, U.K., pp. 425-449.

Gildberg, A., Bogwald, J., Johansen, A., and Stenberg, E. (1996). Isolation of acid peptide fractions from a fish protein hydrolysate with strong stimulatory effect on Atlantic salmon (Salmo salar) head kidney leucocytes. Comp. Biochem. Physiol. 11: 97-101.

Gildberg, A., and Overbo, K. (1990). Purification and characterization of pancreatic elastase from Atlantic cod (Gadus morhua). Comp. Biochem. Physiol. 97B: 775-782.

Gildberg, A., and Raa, J. (1983). Purification and characterization of pepsins from Arctic fish capelin (Mallotus villosus). Comp. Biochem. Physiol. 75A: 337-342.

Gildberg, A., Simpson, B.K., and Haard, N.F. (2000). Uses of enzymes from marine organisms. Seafood Enzymes. Utilization and Influence on Postharvest Seafood Quality. Marcel Dekker, New York, pp. 619-640.

Gill, T.A. (2000). Waste from processing aquatic animals and animal products: implications on aquatic animal pathogen transfer. FAO Fisheries Circular (FAO). .

Gilsenan, P.M., and Ross-Murphy, S.B. (2000). Rheological characterisation of gelatines from mammalian and marine sources. Food Hydrocolloid. 14: 191-195.

Giménez, B., Gómez-Estaca, J., Alemán, A., Gómez-Guillén, M.C., and Montero, M.P. (2009). Physico-chemical and film forming properties of giant squid (Dosidicus gigas) gelatine. Food Hydrocolloid. 23: 585-592.

Giménez, B., Gómez-Guillén, M.C., and Montero, P. (2005). The role of salt washing of fish skins in chemical and rheological properties of gelatine extracted. Food Hydrocolloid. 19: 951-957.

Gimenez, B., Gómez-Guillén, M.C., and Montero, P. (2005). Storage of dried fish skins on quality characteristics of extracted gelatine. Food Hydrocolloid. 19: 958-963.

Gisbert, C., Ros, R., de Haro, A., Walker, D.J., Pilar Bernal, M., Serrano, R., and Avino, J.N. (2003). A plant genetically modified that accumulates $\mathrm{Pb}$ is especially promising for phytoremediation. Biochem. Biophys. Res. Commun. 303: 440-445.

Gjellesvik, D.R. (1991). Fatty acid specificity of bile salt-dependent lipase: Enzyme recognition and super-substrate effects. Biochim. Biophys. Acta, Lipids Lipid Metab. 1086: 167-172.

Gjellesvik, D.R., Lombardo, D., and Walther, B.T. (1992). Pancreatic bile salt dependent lipase from cod, (Gadus morhua): Purification and properties. Biochim. Biophys. Acta, Lipids Lipid Metab. 1124: 123-134.

Gjellesvik, D.R., Lorens, J.B., and Male, R. (1994). Pancreatic carboxylester lipase from Atlantic salmon (Salmo salar). cDNA sequence and computer-assisted modelling of tertiary structure. Eur. J. Biochem. 226: 
603-612.

Goddard, J.S., and Perret, J.S.M. (2005). Co-drying fish silage for use in aquafeeds. Anim. Feed Sci. and Technol. 118: 337-342.

Gogos, C.A., Ginopoulos, P., Salsa, B., Apostolidou, E., Zoumbos, N.C., and Kalfarentzos, F. (1998). Dietary omega-3 polyunsaturated fatty acids plus vitamin $\mathrm{E}$ restore immunodeficiency and prolong survival for severely ill patients with generalized malignancy. Cancer. 82: 395-402.

Goodwin, T.W. (1994). The Biochemistry of the Carotenoids. In: Goodwin, T.W. (Ed.). Chapman and Hall, New York.

Gopakuma, K. (1990). Biochemistry of melanosis in the shellfish and its prevention. Ann. Ind. Fish. Assoc. 7: 17-20.

Govind Rao, M.K., and Achaya, K.T. (1968). Antioxidant activity of squalene. J. Am. Oil Chem. Soc. 45: 296-298.

Goy, R.C., Britto, D.D., and Assis, O.B.G. (2009). A review of the antimicrobial activity of chitosan. Polímeros. 19: 241-247.

Grant, G.A., Sacchettini, J.C., and Welgus, H.G. (1983). A collagenolytic serine protease with trypsin-like specificity from fiddler crab (Uca pugilator). Biochem. 22: 354-356.

Gross, J. (1991). Pigments in Vegetables: Chlorophylls and Carotenoids. Van Nostrand Reinhold, New York.

Gudmundsson, M. (2002). Rheological properties of fish gelatines. Food Eng. Phys. Prop. 67: 2172-2176.

Gudmundsson, M., and Hafsteinsson, H. (1997). Gelatine from cod skins as affected by chemical treatments. J. Food Sci. 62: 37-47.

Guenneugues, P., and lanelli, J. (2014). Surimi resources and market. Surimi and surimi seafood. pp. 25-54.

Guérard, F. (2007). Enzymatic methods for marine by-products recovery. Maximising the value of marine by-products. pp. 107-143.

Guérard, F., and Gal, L.Y. (1987). Characterization of a chymo- sin-like pepsin form the dogfish Scyliorhinus canicula. Comp Biochem Physiol. 88: 823-827.

Guérard, F., and Le Gal, Y. (1989). Electrophoretic study of the caseinolytic activity of a pepsin from the dogfish Scyliorhinus canicula. Biochimie. 71: 767-770.

Guérard, F., Sellos, D., and Gal, Y.L. (2005). Fish and Shellfish Upgrading, Traceability. Adv Biochem Engin/Biotechnol. 96: 127-163.

Guibal, E. (2004). Interactions of metal ions with chitosan-based sorbents: a review. Sep. Purif. Technol. 38(1): 43-74.

Haard, N.F. (1998). Speciality enzymes from marine organisms. Food Technol. 52: 64-67.

Haard, N.F., Dimes, L.E., Arndt, R.E., and Dong, F.M. (1996). Estimation of protein digestibility: IV. Digestive proteinases from the pyloric caeca of Coho salmon (Oncorhynchus kisutch) fed diets containing soybean meal. Comp. Biochem. Physiol. 115B: 533-540.

Haard, N.F., and Simpson, B.K. (1994). Proteases from aquatic organisms and their uses in the seafood industry. Fisheries processing: biotechnological applications. In: Martin, A.M. (Ed.). Chapman and Hall, London, UK, pp. 132-154.

Haddar, A., Sellimi, S., Ghannouchi, R., Alvarez, O.M., Nasri, M., and Bougatef, A. (2012). Functional, antioxidant and film-forming properties of tuna-skin gelatin with a brown algae extract. Int. J. Biol. Macromol. 51(4): 477-483.

Hadley, K.B., Guimont-Desrochers, F., Bailey-Hall, E., Salem Jr, N., YurkoMauro, K., and Field, C.J. (2017). Supplementing dams with both arachidonic and docosahexaenoic acid has beneficial effects on growth and immune development. Prostaglandins Leukot. Essent. Fatty Acids 126: 55-63.

Hadwiger, L.A., Kendra, D.G., Fristensky, B.W., and Wagoner, W. (1981). Chitosan both activated genes in plants and inhibits RNA synthesis in fungi. Chitin in nature and technology. In: Muzzarelli, R.A.A., Jeuniaux, C., and Gooday, G.W. (Ed.). Plenum, New York.

Hadwiger, L.A., Kendra, D.G., Fristensky, B.W., and Wagoner, W. (1981). Chitosan both activated genes in plants and inhibits RNA synthesis in fungi. Chitin in nature and technology. In: Muzzarelli, R.A.A., Jeuniaux, C., and Gooday, G.W. (Ed.). Plenum, New York, pp. 223-230.

Hajjou, M., Smine, A., Guérard, F., and Le Gal, Y. (1995). Purification and some properties of a carboxypeptidase B from dogfish Scyliorhinus canicula. Comp Biochem Physiol 110: 791-798.

Halim, N.R.A., Yusof, H.M., and Sarbon, N.M. (2016). Functional and bioactive properties of fish protein hydolysates and peptides: A comprehensive review. Trends Food Sci. Technol. 51: 24-33.
Hamada, M., Nagai, T., Kai, N., Tanoue, Y., Mae, H., Hashimoto, M. Miyoshi, K., Kumagai, H., and Saeki, K. (1995). Inorganic constituents of bone of fish. Fisheries Sci. 61(3): 517-520.

Hamed, I., Özogul, F., and Regenstein, J.M. (2016). Industrial applications of crustacean by-products (chitin, chitosan, and chitooligosaccharides): A review. Trends Food Sci. Technol. 48: 40-50.

Han, X.Q., and Shahidi, F. (1995). Extraction of harp seal gastric proteases and their immobilization on chitin. Food Chem. 52: 71-76.

Hansen, M.M., Vilstrup, P., and Jensen, N.M. (2002). Patent No. 6,423,346. Washington, DC: U.S. Patent and Trademark Office.

Hargisa, P.S., and Elswyk, M.E.V. (1993). Manipulating the fatty acid composition of poultry meat and eggs for the health conscious consumer. World's Poult. Sci. J. 49: 251-264.

Hashim, P., Ridzwan, M.M., Bakar, J., and Hashim, M.D. (2015). Collagen in food and beverage industries. Int. Food Res. J. 22(1): 1.

Hata, H., Onishi, H., and Machida, Y. (2000). Preparation of CM-chitin microspheres by complexation with iron (III) in w/o emulsion and their biodisposition characteristics in mice. Biomaterials 21(17): 17791788.

Hatton, B., Aao, G.H., Jorgenson, E.H., Storebakken, T., and Goswami, U.C. (1995). Pigmentation of 1-, 2-, and 3-yearold Arctic charr (Salvelinus alpinus) fed on different dietary astaxanthin concentrations. Aquacul. 238: 303-312.

Hauselman, H.J. (2001). Nutripharmaceuticals for osteoarthritis. Best Pract. Res. Clin. Rheumatol. 15: 595-607.

Hayashi, T., Yamaguchi, K., and Konosu, S. (1981). Sensory analysis of taste active component in the extract of boiled snow crab meat. J. Food Sci. 46 (2): 479-483.

Hayes, M. (2018). Marine By-Products as a Source of Proteins for Potential Food, Pharma, and Agricultural Feed Use. Novel Proteins for Food Pharmaceuticals, and Agriculture, and Agriculture: Sources, Applications, and Advances. p. 163.

Hayes, M., Carney, B., Slater, J., and Brück, W. (2008a). Mining marine shellfish wastes for bioactive molecules: Chitin and chitosan ndash Part A: extraction methods. Biotechnol. J. Healthc. Nutr. Technol. 3(7): 871-877.

Hayes, M., Carney, B., Slater, J., and Brück, W. (2008b). Mining marine shellfish wastes for bioactive molecules: chitin and chitosan-Part B: applications. Biotechnol. J. Healthc. Nutr. Technol. 3(7): 878-889.

Hazaveh, P., Nafchi, A.M., and Abbaspour, H. (2015). The effects of sugars on moisture sorption isotherm and functional properties of cold water fish gelatin films. Int. J. Biol. Macromol. 79: 370-376.

He, H., Chen, X., Sun, C., Zhang, Y., and Gao, P. (2006). Preparation and functional evaluation of oligopeptide-enriched hydrolysate from shrimp (Acetes chinensis) treated with crude protease from Bacillus sp. SM98011. Bioresour. Technol. 97(3): 385-390.

He, X., Yang, X., and Guo, Y. (2007). Effects of different dietary oil sources on immune function in cyclophosphamide immunosuppressed chickens. Anim. Feed Sci. Technol. 139: 186-200.

He, Y., and Shahidi, F. (1997). Enzymatic esterification of $\omega$-3 fatty acid concentrates from seal blubber oil with glycerol. J. Am. Oil Chem. Soc. 75: 945-951.

Helland, I.B., Saugstad, O.D., Smith, L., Saarem, K., Solvoll, K., Ganes, T., and Drevon, C.A. (2001). Similar effects on infants of $n-3$ and $n-6$ fatty acids supplementation to pregnant and lactating women. Pediat. 108: E82.

Hennessey, J.P. Jr, and Siebenaller, J.F. (1987). Pressure-adaptive differences in proteolytic inactivation of M-lactate dehydrogenase homologues from marine fishes. J, exp. Zool. 241: 9-15.

Hernández-Ledesma, B., and Herrero, M. (Ed.). (2013). Bioactive compounds from marine foods: plant and animal sources. John Wiley and Sons.

Hernández-Briones, A., Velázquez, G., Vázquez, M., and Ramírez, J.A. (2009). Effects of adding fish gelatine on Alaska pollock surimi gels. Food Hydrocolloid. 23: 2446-2449.

Hicks, T.M., and Verbeek, C.J.R. (2016). Meat industry protein by-products: sources and characteristics. Protein Byproducts. Academic Press, pp. 37-61.

Higashi, K., Okamoto, Y., Mukuno, A., Wakai, J., Hosoyama, S., Linhardt, R.J., and Toida, T. (2015). Functional chondroitin sulfate from Enteroctopus dofleini containing a 3-O-sulfo glucuronic acid residue. 
Carbohydr. Polym. 134: 557-565.

Hilditch, T.P., and Williams, P.N. (1964). The Chemical Constitution of Natural Fats. Chapman and Hall, London, pp. 34-79.

Hirano, S., and Midorikawa, T. (1998). Chitin and chitosan: specific interactions, thermal properties and crystallization behaviour. Biomater. 19: 293-297.

Hirano, S., and Nagao, N. (1989). Effects of chitosan, pectic acid, lysozyme, and chitinase on the growth of several phytopathogens. Carbohydr. Res. 53: 3065-3066.

Hirano, S., and Noishiki, Y. (1985). The blood compatibility of chitosan and $\mathrm{N}$-acylchitosans. J. Biomed. Mater. Res. 19(4): 413-417.

Hoang, Q.Q., Sicheri, F., Howard, A.J., and Yang, D.S.C. (2003). Bone recognition mechanism of porcine osteocalcin from crystal structure. Nature. 425: 977-980.

Hochberg, M.C., Martel-Pelletier, J., Monfort, J., Möller, I., Castillo, J.R., Arden, N., and and Henrotin, Y. (2016). Combined chondroitin sulfate and glucosamine for painful knee osteoarthritis: a multicentre, randomised, double-blind, non-inferiority trial versus celecoxib. Ann. Rheum. Dis. 75(1): 37-44.

Holmer, G. (1989). Triglycerides. Marine Biogenic lipids, fats, and oils. In: Ackman, R.G. (Ed.). pp. 139-173.

Holzer, D. (1996). Gelatine production. US patent 5,484,888.

Hon, D.N.S. (1996). Chitin and chitosan: medical applications. Polysaccharides in Medical Applications. In: Dumitriu, S. (Ed.). Marcel Dekker, New York, pp. 631-649.

Hongpattarakere, T., and Riyaphan, O. (2008). Effect of deacetylation conditions on antimicrobial activity of chitosans prepared from carapace of black tiger shrimp. Songklanakarin J. Sci. Technol. 30(1):

Horowitz, S., Roseman, S., and Blumenthal, H.J. (1957). The preparation of glucosamine oligosaccharides. I. Separation. J. Am. Chem. Soc. 79: 5046-5049.

Hosseinnejad, M., and Jafari, S.M. (2016). Evaluation of different factors affecting antimicrobial properties of chitosan. Int. J. Biol. Macromol. 85: 467-475.

Howling, G.I., Dettmar, P.W., Goddard, P.A., Hampson, F.C., Dornish, M., and Wood, E.J. (2001). The effect of chitin and chitosan on the proliferation of human skin fibroblasts and keratinocytes in vitro. Biomaterials. 22(22): 2959-2966.

Hu, F.Q., Chen, W.W., Zhao, M.D., Yuan, H., and Du, Y.Z. (2013). Effective antitumor gene therapy delivered by polyethylenimine-conjugated stearic acid-g-chitosan oligosaccharide micelles. Gene Ther. 20(6): 597.

Hua, J., Sakamoto, K., Kikukawa, T., Abe, C., Kurosawa, H., and Nagaoka, I. (2007). Evaluation of the suppressive actions of glucosamine on the interleukin-1 $\beta$-mediated activation of synoviocytes. Inflamm. Res. 56(10): 432-438.

Huang, T., Tu, Z.C., Shangguan, X., Wang, H., Sha, X., and Bansal, N. (2018). Rheological behavior, emulsifying properties and structural characterization of phosphorylated fish gelatin. Food Chem. 246: 428-436.

Huang, T., Tu, Z.C., Wang, H., Liu, W., Zhang, L., Zhang, Y., and ShangGuan, X.C. (2017). Comparison of rheological behaviors and nanostructure of bighead carp scales gelatin modified by different modification methods. J. Food Sci. Technol. 54(5): 1256-1265.

Huang, X., Huang, X., Jiang, X.H., Hu, F.Q., Du, Y.Z., Zhu, Q.F., and Jin, C.S. (2012). In vitro antitumour activity of stearic acid-g-chitosan oligosaccharide polymeric micelles loading podophyllotoxin. J. Microencapsulation 29(1): 1-8.

Hultin, H.O., and Kelleher, S.D. (2000). Surimi processing from dark muscle fish. Surimi and Surimi Seafood. In: Park, J.W. (Ed.). Marcel Dekker, Inc., New York, NY, pp. 59-77.

Hultin, H.O., and Kelleher, S.D. (1999). Protein composition isolated from a muscle source. U.S. patent 6,005,073. U.S. Patent and Trademark Office.

Hwang, D. (2000). Fatty acids and immune responses: A new perspective in searching for clues to mechanism. Annu. Rev. Nutr. 20: 431-456.

lijima, N., Tanaka, S., and Ota, Y. (1998). Purification and characterization of bile salt-activated lipase from the hepatopancreas of red sea bream, Pagrus major. Fish Physiol. Biochem. 18: 59-69.

Ikeda, I., Sugano, M., Yoshida, K., Sasaki, E., Iwamoto, Y., and Hatano, K. (1993). Effects of chitosan hydrolysates on lipid absorption and on serum and liver lipid concentration in rats. J. Agri. Food Chem. 41:
431-435

Imm, J.Y., and Lee, C.M. (1999). Production of Seafood Flavour from Red Hake (Urophycis chuss) by Enzymatic Hydrolysis. J. Agric. Food Chem. 47: 2360-2366.

Irawan, C., Nata, I.F., Putra, M.D., Marisa, R., Asnia, M., and Arifin, Y.F. (2018). Biopolymer of Chitosan from Fish Scales as Natural Coagulant for Iron-Contaminated Groundwater Treatment. J. Rekayasa Kim. Lingkung. 13(2): 93-99.

Ishii, H., Minegishi, M., Lavitpichayawong, B., and Mitani, T. (1995). Synthesis of chitosan-amino acid conjugates and their use in heavy metal uptake. Int. J. Biol. Macromol. 17(1): 21-23.

Ishimura, G., and Bailey, M. (2013). The market value of freshness: observations from the swordfish and blue shark longline fishery. Fishe. Sci. 79(3): 547-553.

Islam, R., and Datta, B. (2015). Diversity of chitinases and their industrial potential. Int. J. Appl. Res. 1: 55-60.

Itabashi, Y., and Ota, T. (1994). Lipase activity in scallop hepatopancreas. Fish. Sci. 60: 347

Ito, M., Matahira, Y., and Sakai, K. (1998). Kichin, Kitosan Kenkyu. Publ. Nippon Kichin, Kitosan Gakkai. 4: 142.

Izzo, H.V., and Ho, C.T. (1992). Peptide-specific Maillard reaction products: a new pathway for flavor chemistry. Trends Food Sci. Technol. 3: 253-257.

Jaczynski, J. (2008). Protein and Lipid Recovery from Food Processing ByProducts Using Isoelectric Solubilization/Precipitation. Food Chem Research Developments. In: Papadopoulos, K.N. (Ed.). Nova Science Publishers, Inc., pp. 167-198.

Jafar, S.S., Hultin, H.O., Bimbo, A.P., Crowther, J.B., and Barlow, S.M. (1994). Stabilization by antioxidants of mayonnaise made from fish oil. J. Food Lipids 1(4): 295-311.

Jameela, S.R., Misra, A., and Jayakrishnan, A. Drug delivery systems based on inorganic materials: I. Synthesis and characterization of a zeolitecyclophosphamide system. J. Biomater. Sci. Polymer Ed. 6: 621-632.

Jamilah, B., and Harvinder, K.G. (2002). Properties of gelatines from skins of fish-black tilapia (Oreochromis mossambicus) and red tilapia (Oreochromis nilotica). Food Chem. 77: 81-84.

Jannat-Alipour, H., Rezaei, M., Shabanpour, B., and Tabarsa, M. (2019). Edible green seaweed, Ulva intestinalis as an ingredient in surimi-based product: chemical composition and physicochemical properties. J. Appl. Phycol. 1-11.

Jayasinghe, C., Gotoh, N., Tokairin, S., Ehara, H., and Wada, S. (2003). Inter species changes of lipid compositions in liver of shallow water sharks from the Indian Ocean. Fisheries Sci. 69: 644-653.

Je, J.Y., Cho, Y.S., and Kim, S.K. (2006). Characterization of (Aminoethyl) chitin/DNA Nanoparticle for Gene Delivery. Biomacromol. 7: 34483451.

Je, J.Y., and Kim, S.K. (2006). Antimicrobial action of novel chitin derivative. Biochim. et Biophys. Acta. 1760: 104-109.

Je, J.Y., and Kim, S.K. (2006). Antioxidant activity of novel chitin derivative. Bioorg. Med. Chem. Lett. 16: 1884-1887.

Je, J.Y., and Kim, S.K. (2006). Dicationic pyridium porphyrins appending different peripheral substituents: synthesis and studies for their interaction with DNA. Biochim. Biophys. Acta 1170: 104-109.

Je, J.Y., Kim, S.Y., and Kim, S.K. (2005). Preparation and antioxidative activity of hoki frame protein hydrolysate using ultrafiltration membranes. Eur. Food Res. Technol. 221: 157-162.

Je, Y., Park, P.J., and Kim, S.K. (2005). Antioxidant activity of a peptide isolated from Alaska pollack (Theragra chalcogramma) frame protein hydrolysate. Food Res. Int. 38: 45-50.

Jensen, C.L. (2006). Effects of $n-3$ fatty acids during pregnancy and lactation. Am. J. Clin. Nutr. 83: S1452-1457S.

Jensen, R.G. (1983). Detection and determiantion of lipase (acylglycerol hydrolase) activity from various sources. Lipids. 18: 650-657.

Jeon, Y.-J., Kamil, J.Y.V.A., and Shahidi, F. (2002). Chitosan as an edible invisible film for quality preservation of herring and atlantic cod. J. Agri. Food Chem. 50: 5167-5178.

Jeon, Y.-J., Shahidi, F., and Kim, S.-K. (2000). Preparation of chitin and chitosan oligomers and their applications in physiological functional foods. Food Rev. International. 16: 159-176.

Jeon, Y.J., and Kim, S.K. (2002). Antitumor activity of chitosan oligosaccharides produced in ultrafiltration membrane reactor system. J. Micro- 
biol. Biotechnol. 12: 503-507.

Jeon, Y.J., and Kim, S.K. (2000). Continuous production of chitooligosaccharides using a dual reactor system. Process Biochem. 35: 623-632.

Jeon, Y.J., Park, P.J., and Kim, S.K. (2001). Antimicrobial effect of chitooligosaccharides produced by bioreactor. Carbolyd. Polym. 44: 71-76.

Jeppesen, C., Bjerregaard, P., and Jørgensen, M.E. (2014). Dietary patterns in Greenland and their relationship with type 2 diabetes mellitus and glucose intolerance. Public Health Nutr. 17(2): 462-470.

Johnson, E.L., and Peniston, Q.P. (1982). Utilization of Shellfish Waste for Chitin, Chitosan Production. Chemistry and Biochemistry of Marine Food Products. In: Martin, R.E., Flick, G.J., Hebard, C.E., and Ward, D.R. (Ed.). AVI Publishing Co., Westport, CT, USA, pp. 415-428.

Jongjareonrak, A., Benjakul, S., Visessanguan, W., Prodpran, T., and Tanaka, M. (2006a). Characterization of edible films from skin gelatine of brownstripe red snapper and bigeye snapper. Food Hydrocolloid. 20: 492-501.

Jongjareonrak, A., Benjakul, S., Visessanguan, W., and Tanaka, M. (2006b). Skin gelatine from bigeye snapper and brownstripe red snapper: chemical compositions and effect of microbial transglutaminase on gel properties. Food Hydrocolloid. 20: 1216-1222.

Ju, S.-.J., and Harvey, H.R. (2004). Lipids as markers of nutritional condition and diet in the Antarctic krill Euphausia superba and Euphausia crystallorophias during austral winter. Deep-sea Res. II. 51: 2199-2214.

Jun, S.Y., Park, P.J., Jung, W.K., and Kim, S.K. (2004). Purification and characterization of an antioxidative peptide from enzymatic hydrolysate of yellowfin sole (Limanda aspera) frame protein. Eur. Food Res. Technol. 219: 20-26.

Jung, W.K., and Kim, S.K. (2007). Calcium-binding peptide derived from pepsinolytic hydrolysates of hoki (Johnius belengerii) frame. Eur. Food Res. Technol. 224: 763-767.

Jung, W.K., Lee, B.J., and Kim, S.K. (2006). Fish-bone peptide increases calcium solubility and bioavailability in ovariectomised rats. Brit. J. Nutr. 95: 124-128.

Jung, W.K., Park, P.J., Byun, H.G., Moon, S.H., and Kim, S.K. (2005). Preparation of hoki (Johnius belengerii) bone oligophosphopeptide with a high affinity to calcium by carnivorous intestine crude proteinase. Food Chem. 91: 333-340.

Kadam, S.U., and Prabhasankar, P. (2010). Marine foods as functional ingredients in bakery and pasta products. Food Res. Int. 43(8): 19751980

Kailasapathy, K., and Salampessy, J. (1999). Suitability of shark flesh for surimi production. Food Australia. 5: 110-115.

Kaiya, A. (1990). The use of natural squalene and squalane and the latest situation of the raw materials. J. Japan oil Chem. Soc. 39: 525-529.

Kamer, D.D.A., Palabiyik, I., Işık, N.O., Akyuz, F., Demirci, A.S., and Gumus, T. (2019). Effect of confectionery solutes on the rheological properties of fish (Oncorhynchus mykiss) gelatin. LWT 101: 499-505.

Kamil, J.Y.V.A., Jeon, Y.-J., and Shahidi, F. (2002). Antioxidative activity of chitosans of different viscosity in cooked comminuted flesh of herring (Clupea harengus). Food Chem. 79: 69-77.

Kanauchi, O., Deuchi, K., Imasato, Y., Shizukuishi, M., and Kobayashi, E. (1995). Mechanism for the inhibition of fat digestion by chitosan and for the synergistic effect of ascorbate. Biosci. Biotech. Biochem. 59: 786-790.

Kang, K.T., Heu, M.S., and Kim, J.S. (2007). Preparation and food component characteristics of snack using flatfish-frame. J. Korean Soc. Food Sci. Nutr. 36(5): 651-656.

Kaplan, D.L., Mayer, J.M., Ball, D., McCassie, J., Allen, A.L., and Stenhouse, P. (1993). Fundamental of biodegradable polymer. Biodegradable Polymers and Packaging. In: Ching, C., Kaplan, D., and Thomas, E. (Ed.). Technomic Publishing, Lancaster, pp. 1-42.

Karaman, S., Cengiz, E., Kayacier, A., and Dogan, M. (2016). Exposure to air accelerates the gelation of gelatin: Steady and dynamic shear rheological characterization to see the effect of air on the strength of gelatin gel. Int. J. Food Prop. 19(4): 721-730.

Karayannakidis, P.D., and Zotos, A. (2016). Fish processing by-products as a potential source of gelatin: a review. J. Aquat. Food Prod. Technol. 25(1): 65-92.

Karayannakidis, P.D., Zotos, A., Petridis, D., and Taylor, K.D.A. (2008). Physicochemical changes of sardines (Sardina pilchardus) at $-18^{\circ} \mathrm{C}$ and functional properties of Kamaboko gels enhanced with $\mathrm{Ca} 2+$ ions and
MTGase. J. Food Process Eng. 31: 372-97.

Karim, A.A., and Bhat, R. (2009). Fish gelatine: properties, challenges, and prospects as an alternative to mammalian gelatines. Food Hydrocolloid. 23: 563-576.

Karim, A.A., and Bhat, R. (2008). Gelatin alternatives for the food industry: recent developments, challenges and prospects. Trends Food Sci. Technol. 19(12): 644-656.

Kasankalaa, L.M., Xuea, Y., Weilonga, Y., Honga, S.D., and He, Q. (2007) Optimization of gelatine extraction from grass carp (Catenopharyngodon idella) fish skin by response surface methodology. Bioresour. Technol. 98: 3338-3343.

Kelley, D.S. (2001). Modulation of human immune and inflammatory responses by dietary fatty acids. Nutrition. 17: 669-673.

Kendra, D.F., and Hadwiger, L.A. (1984). Characterization of the smallest chitosan oligomer that is maximally antifungal to Fusarium solani and elicits pisatin formation in Pisum sativum. Exp. Mycol. 8: 276-281.

Kennedy, J.F., and Cabral, J.M.S. (1983). Immobilized enzymes. Solid phase biochemistry. Analytical and synthetic aspects. In: Scouten, W.H. (Ed.). John Wiley and Sons, New York, pp. 253-391.

Kerry, J.P., and Murphy, S.C. (2007). Physical and chemical properties of lipid by-products from seafood waste. Maximising the value of marine by-products. Woodhead Publishing, pp. 22-46.

Kestin, S. (2017). Sustainability and fish welfare. The Meat Crisis: Developing more Sustainable and Ethical Production and Consumption.

Khor, E., and Lim, L.Y. (2003). Implantable Applications of Chitin and Chitosan. Biomater. 24: 2339-2349.

Kilara, A. (1985). Enzyme-modified protein food ingredients. Process Biochem. 20: 149-158.

Kim, J.S., and Park, J.W. (2004). Characterization of acid-soluble collagen from Alaska pollock surimi processing by-products. J. Food Sci. 69 C637-C642.

Kim, J.S., Shahidi, F., and Heu, M.S. (2003). Characteristics of salt-fermented sauces from shrimp processing byproducts. J. Agric. Food Chem. 51: 784-92.

Kim, S.-K., Kim, Y.-T., Byun, H.-G., Nam, K.-S., Joo, D.-S., and Shahidi, F. (2001). Isolation and characterization of antioxidative peptides from gelatine hydrolysate of Alaska Pollack skin. J. Agric.Food Chem. 49 1984-1989.

Kim, S.-K., Park, P.J., Kim, J.B., and Shahidi, F. (2002). Purification and Characterization of a Collagenolytic Protease from the Filefish, Novoden modestrus. J. Biochem. Mol. Biol. 35: 165-171.

Kim, S.K., Choi, J.S., Lee, C.K., Byun, H.G., Jeon, Y.J., and Lee, E.H. (1998). Synthesis and biocompatibility of the hydroxyapatite ceramic composites from tuna bone (III)-SEM photographs of bonding properties hydroxyapatite ceramic composites in the simulated body fluid. J. Korean Indus. Eng. Chem. 9: 322-329.

Kim, S.K., Choi, J.S., Lee, C.K., Byun, H.G., Jeon, Y.J., and Lee, E.H.J. (1997) Synthesis and biocompatibility of the hydroxyapatite ceramic composites from tuna bone (II)-The sintering properties of hydroxyapatite treated with wet milling process. J. Korean Indus. Eng. Chem. 8: 1000-1005.

Kim, S.K., Choi, Y.R., Park, P.J., Choi, J.H., and Moon, S.H. (2000). Screening of biofunctional peptides from cod processing wastes. J. Korean Soc. Agric. Chem. Biotechnol. 43: 225-227.

Kim, S.K., Jeon, Y.J., Byun, H.G., Park, P.J., Kim, G.H, Choi, Y.R, and Lee, Y.S. (1999). Calcium absorption acceleration effect on phosphorylated and non-phosphorylated peptides from hoki (Johnius belengeri) frame. J. Korean Fisheries Soc. 32: 713-717.

Kim, S.K., and Jung, W.K. (2007). Fish and bone as a calcium source. Maximizing the value of marine by-products. In: Shahidi, F. (Ed.). CRC Press, Boca Raton, FL, pp. 328-336.

Kim, S.K., Lee, H.C., Byun, H.G., and Jeon, Y.J. (1996). Isolation and characterization of antioxidative peptides from enzymatic hydrolysates of yellowfin sole skin gelatin. Korean J. Fish Aquat. Sci. 29(2): 246-255.

Kim, S.K., and Mendis, E. (2006). Bioactive compounds from marine processing by-products - a review. Food Res. Int. 39: 383-393.

Kim, S.K., and Park, P.J. (2000). Evaluation of mucous membrane irritation by hydroxyapatite sinter produced from tuna bone in Syrian hamsters. Korean J. Life Sci. 10: 605-609.

Kim, S.K., Park, P.J., Byun, H.G., Je, J.Y., Moon, S.H., and Kim, S.H. (2003) Recovery of fish bone from hoki (Johnius belengerii) frame using 
proteolytic enzyme isolated from mackerel intestine. J. Food Biochem. 27: 255-266.

Kim, S.K., Park, P.J., and Kim, Y.T. (2001). Study on acute subcutaneous toxicity of hydroxyapatite sinter produced from tuna bone in SpragueDawly rats. Korean J. Life Sci. 11: 97-102.

Kim, S.K., Perera, U.M.S.P., Rajapakse, N., and Kim, S. (2016). Seafood Processing By-Products. Springer-Verlag, New York.

Kim, S.K., and Rajapakse, N. (2005). Enzymatic production and biological activities of chitosan oligosaccharides (COS): A review. Carbohydr. Polym. 62: 357-368.

Kim, S.Y., Je, J.Y., and Kim, S.K. (2007). Purification and characterization of antioxidant peptide from hoki (Johnius belengerii) frame protein by gastrointestinal digestion. J. Nutr. Biochem. 18: 31-38.

Kimura, S., and Ohno, Y. (1987). Fish type I collagen: Tissue-specific existence of two molecular forms, $(\alpha 1) 2 \alpha 2$ and $\alpha 1 \alpha 2 \alpha 3$, in alaska pollack. Comp. Biochem. Physiol. 88(2): 409-413.

Kirti, K., Amita, S., Priti, S., Mukesh Kumar, A., and Jyoti, S. (2014). Colorful world of microbes: carotenoids and their applications. Adv. Biol. 2014: 837891.

Kitsugi, T., Yamamuro, T., Nakamura, T., Kotani, S., Kokubo, T., and Takeuchi, H. (1993). Four calcium phosphate ceramics as bone substitutes for non-weight bearing. Biomater. 14: 216-224.

Kittiphattanabawon, P., Benjakul, S., Visesanguan, W., Nagai, T., and Tanaka, M. (2005). Characterization of acid-soluble collagen from skin and bone of bigeye snapper (Priacanthus tayenus). Food Chem. 89: 363-372.

Kittiphattanabawon, P., Benjakul, S., Visessanguan, W., and Shahidi, F. (2010). Isolation and characterization of collagen from the cartilages of brownbanded bamboo shark (Chiloscyllium punctatum) and blacktip shark (Carcharhinus limbatus). LWT-Food Sci. Technol. 43(5): 792-800.

Klompong, V., Benjakul, S., Kantachote, D., and Shahidi, F. (2007). Antioxidative activity and functional properties of protein hydrolysate of yellow stripe trevally (Selaroides leptolepis) as influenced by the degree of hydrolysis and enzyme type. Food Chem. 102: 1317-1327.

Knorr, D. (1991). Recovery and utilization of chitin and chitosan in food processing waste management. Food Technol. 45: 114-122.

Kołodziejska, I., Kaczorowski, K., Piotrowska, B., and Sadowska, M. (2004). Modification of the properties of gelatine from skins of Baltic cod (Gadus morhua) with transglutaminase. Food Chem. 86: 203-209.

Kołodziejska, I., Skierka, E., Sadowska, M., Kołodziejski, W., and Niecikowska, C. (2008). Effect of extracting time and temperature on yield of gelatine from different fish offal. Food Chem. 107: 700-706.

Kolodzjieska, I., Kaczorowski, K., Piotrowsia, B., and Sadowska, M. (2004). Modification of properties of gelatine from skins of Baltic cod (Gadus morhua) with transglutaminase. Food Chem. 86: 203-209.

Komai, T., Kawabata, C., Tojo, H., Gocho, S., and Ichishima, E. (2007). Purification of serine carboxypeptidase from the hepatopancreas of Japanese common squid Todarodes pacificus and its application for elimination of bitterness from bitter peptides. Fish. Sci. 73(2): 404-411.

Konieczka, P., Czauderna, M., and Smulikowska, S. (2017). The enrichment of chicken meat with omega-3 fatty acids by dietary fish oil or its mixture with rapeseed or flaxseed-Effect of feeding duration: Dietary fish oil, flaxseed, and rapeseed and $n-3$ enriched broiler meat. Anim. Feed Sci. Technol. 223: 42-52.

Kono, M., Wider, M.N., Matsui, T., Furukawa, K., Koga, D., and Aida, K. (1995). Chitinolytic enzyme activities in the hepatopancreas, tail fan and hemolymph of kuruma prawn Penaeus japonicus during the moult cycle. Fish Sci. 61: 727-728.

Konosu, S. (1979). The taste of fish and shellfish. Food Taste Chemistry. In: Boudreau, JC (Ed.). American Chemical Society, 115: pp. 185-203.

Kouba, M., and Mourot, J. (2011). A review of nutritional effects on fat composition of animal products with special emphasis on $\mathrm{n}-3$ polyunsaturated fatty acids. Biochimie 93(1): 13-17.

Koyama, Y.I., Hirota, A., Mori, H., Takahara, H., Kuwaba, K., Kusubata, M., Matsubara, Y., Kasugai, S., Itoh, M., and Irie, S. (2001). Ingestion of gelatin has differential effect on bone mineral density and body weight in protein undernutrition. J. Nutr. Sci. Vitaminol. 47(1): 84-86.

Kozo, F. (1997). Extraction of astaxanthin from shell of lobster or shrimp or crab and apparatus therefore. Japanese Patent No. JP 9301950A.

Krajewska, B. (2004). Application of chitin- and chitosan-based materials for enzyme immobilizations: a review. Enzyme Microb. Technol. 35: 126-139.

Krinsky, N.I. (1994). The biological properties of carotenoids. Pure Appl. Chem. 66(5): 1003-1010.

Kristinsson, H.G. (2007). Aquatic food protein hydrolysates. Maximizing the value of marine by-products. In: Shahidi, F. (Ed.). CRC Press, Boca Raton, FL, pp. 229-248.

Kristinsson, H.G., and Rasco, B.A. (2000). Hydrolysis of salmon muscle proteins by an enzyme mixture extracted from Atlantic salmon (Salmo Salar) Pyloric Caeca. J. Food Biochem. 24: 177-187.

Kristjansson, M.M., Gudmundsdottir, S., Fox, J.W., and Bjarnason, J.B. (1995). Characterization of collagenolytic serine proreinase from the Atlantic cod (Gadus morhua). Comp. Biochem. Physiol. 110B: 707-717.

Kudryashova, E.V., Suhoverkov, K.V., and Sokolov, N.N. (2015). PEG-chitosan branched copolymers to improve the biocatalytic properties of Erwinia carotovora recombinant L-asparaginase. Biomed Khim. 61(4): 480-487.

Kumar, M.N.V.R. (2000). A review of chitin and chitosan applications. React. Funct. Polym. 46: 1-27.

Kurita, K. (2001). Controlled functionalization of the polysaccharide chitin. Prog. Polym. Sci. 26(9): 1921-1971.

Kurita, K., Tomita, K., Ishii, S., Nishimura, S., and Shimoda, K. (1993) $\beta$-chitin as a convenient starting material for acetolysis for efficient preparation of $\mathrm{N}$-acetylchitooligosaccharides. J. Poly. Sci.: Part A: Poly. Chem. 31: 2393-2395.

Kurita, K., Tomita, K., Tada, T., Ishii, S., Nishimura, S.I., and Shimoda, K. (1993). Squid chitin as a potential alternative chitin source: Deacetylation behavior and characteristic properties. J. Polym. Sci. Part A: Polym. Chem. 31: 485.

Kurtovic, I., and Marshall, S.N. (2013). Potential of fish by-products as a source of novel marine lipases and their uses in industrial applications. Lipid Technol. 25(2): 35-37.

Kurtovic, I., Marshall, S.N., Zhao, X., and Simpson, B.K. (2009). Lipases from Mammals and Fishes. Reviews in Fisheries Science. 17: 18-40.

Kuzan, A., Smulczyńska-Demel, A., Chwiłkowska, A., Saczko, J., Frydrychowski, A., and Dominiak, M. (2015). An estimation of the biologica properties of fish collagen in an experimental in vitro study. Adv. Clin. Exp. Med 24(3): 385-392.

Kwak, H.W., Kim, J.E., and Lee, K.H. (2019). Green fabrication of antibacterial gelatin fiber for biomedical application. React. Funct. Polym. 136: 86-94.

Kwak, H.W., Shin, M., Lee, J.Y., Yun, H., Song, D.W., Yang, Y., Shin, B.S., Park Y.H., and Lee, K.H. (2017). Fabrication of an ultrafine fish gelatin nanofibrous web from an aqueous solution by electrospinning. Int. J. Biol. Macromol. 102: 1092-1103.

Landsberg, G.M., Mougeot, I., Kelly, S., and Milgram, N.W. (2015). Assessment of noise-induced fear and anxiety in dogs: modification by a novel fish hydrolysate supplemented diet. J. Vet. Behav. 10(5): 391-398.

Langasco, R., Cadeddu, B., Formato, M., Lepedda, A.J., Cossu, M., Giunchedi, P., and and Gavini, E. (2017). Natural collagenic skeleton of marine sponges in pharmaceutics: Innovative biomaterial for topical drug delivery. Mater. Sci. Eng., C 70: 710-720.

Larrazábal, M.J., and Camacho, M.M. (2008). Characteristics of the salmon marinated in dry and for immersion in osmotic solution. Ciencia $Y$ Tecnología Alimentaria. 6: 20-26.

Larsen, R., Olsen, S.H., Kristoffersen, S., and Elvevoll, E.O. (2008). Low salt brining of pre-rigor filleted farmed cod (Gadus morhua L.) and the effects on different quality parameters. LWT-Food Science and Technology 41(7): 1167-1172.

Larsen, T., Thilsted, S.H., Kongsbak, K., and Hansen, M. (2000). Whole small fish as a rich calcium source. Br. J. Nutr. 83: 191-196.

Lawrence, R., and Sorrell, T. (1993). Eicosapentaenoic acid in cystic fibrosis: Evidence of a pathogenetic role for leukotriene B4. Lancet. 342 : 465-469.

Le Gouic, A.V., Harnedy, P.A., and FitzGerald, R.J. (2019). Bioactive Peptides from Fish Protein By-Products. Bioactive Molecules in Food 355-388.

Le Poncin, M. (1996a). Experimental study: stress and memory. Eur Neuropsychopharmacol. 6: 110-112.

Le Poncin, M. (1996b). Nutrient presentation of cognitive and memory 
performances. Eur Neuropsychopharmacol. 6: 187-P19-4.

Lee, C.M. (2007). Seafood flavour from processing by-products. Maximizing the value of marine by-products. In: Shahidi, F. (Ed.). CRC Press, Boca Raton, FL, pp. 304-327.

Lee, C.M. (1994). Surimi processing from lean fish. Seafoods: Chemistry, Processing Technology and Quality. In: Shahidi, F., and Botta, J.R. (Ed.). Blackie and Academic Professional, London, U.K., pp. 263-287.

Lee, H., Kim, T.H., and Park, T.G.J. (2002). A Receptor-mediated Gene Delivery System Using Streptavidin and Biotin-derivatized, Pegylated Epidermal Growth Factor. Controlled Release. 83: 109-119.

Lee, R.F., Nevenzel, J.C., and Paffenhofer, G.A. (1970). Wax esters in marine copepods. Sci. 167: 1510-1511.

Leger, C., Bauchart, D., and Flanzy, J. (1977). Some properties of pancreatic lipase in Salmo gairdnerii Rich: $\mathrm{Km}$, effects of bile salts and $\mathrm{Ca}^{2+}$, gel filtrations. Comp. Biochem. Physiol., Part B: Biochem. Mol. Biol. 57 359-363.

Lesnierowski, G., and Stangierski, J. (2018). What's new in chicken egg research and technology for human health promotion? A review. Trends Food Sci. Technol. 71: 46-51.

Leuenberger, B.H. (1991). Investigation of viscosity and gelation properties of different mammalian and fish gelatines. Food Hydrocolloid. 5: 353-361.

Li, F., Shetty, A.K., and Sugahara, K. (2007). Neuritogenic activity of chondroitin/dermatan sulfate hybrid chains of embryonic pig brain and their mimicry from shark liver involvement of the pleiotrphin and hepatocyte growth factor signling pathways. J. Biol. Chem. 282(5): 2956-2966.

Li, H., Tyndale, S.J., Heath, D., and DandLetcher, R.J. (2005). Determination of carotenoids and all-trans-retinol in fish eggs by liquid chromatography-elctrospray ionization-tandem mass spectrometry. J. Chromatogr. 816: 49-56.

Li, J., Yuang, X., and Guo, S. (2008). Studies on the Storage Experiment and Prediction of Shelf-life of Fishbone Snack Food. Food Ferment. Ind. 6: .

Li, X.F., Feng, X.Q., Yang, S., Fu, G.Q., Wang, T.P., and Su, Z.X. (2010). Chitosan kills Escherichia coli through damage to be of cell membrane mechanism. Carbohydr. Polym. 79(3): 493-499.

Liaset, B., Julshamn, K., and Espe, M. (2003). Chemical composition and theoretical nutritional evaluation of the produced fractions from enzymic hydrolysis of salmon frames with Protamex ${ }^{\mathrm{TM}}$. Process Biochem. 38(12): 1747-1759.

Lie, O., and Lambertsen, G. (1985). Digestive lipolytic enzymes in cod (Gadus morhua): fatty acid specificity. Comp. Biochem. Physiol. 80B 447-450.

Lignot, B., Lahogue, V., and Bourseau, P. (2003). Enzymatic extraction of chondroitin sulfate from skate cartilage and concentration-desalting by ultrafiltration. J. Biotechnol. 103: 281-284.

Lin, K., Zhang, D., Macedo, M.H., Cui, W., Sarmento, B., and Shen, G. (2019). Advanced Collagen-Based Biomaterials for Regenerative Biomedicine. Adv. Funct. Mater. 29(3): 1804943.

Lin, L., and Li, B.F. (2006). Radical scavenging properties of protein hydrolysates from Jumbo flying squid (Dosidicus eschrichitii Steenstrup) skin gelatin. J. Sci. Food Agric. 86(14): 2290-2295.

Lin, L., Regenstein, J.M., Lv, S., Lu, J., and Jiang, S. (2017). An overview of gelatin derived from aquatic animals: Properties and modification. Trends Food Sci. Technol. 68: 102-112.

Lippi, G., Targher, G., and Franchini, M. (2010). Vaccination, squalene and anti-squalene antibodies: facts or fiction? European J. Intern. Med. 21(2): 70-73.

Liu, D., and Denbow, D.M. (2001). Maternal dietary lipids modify composition of bone lipids and ex vivo prostaglandin E2 production in early postnatal Japanese quail. Poult. Sci. 80: 1344-1352.

Liu, D., Nikoo, M., Boran, G., Zhou, P., and Regenstein, J.M. (2015). Collagen and gelatin. Annu. Rev. Food Sci. Technol. 6: 527-557.

Liu, H., Li, D., and Guo, S. (2008). Rheological properties of channel catfish (Ictalurus punctaus) gelatine from fish skins preserved by different methods. LWT - Food Sci. Technol. 41: 414-419.

Liu, M., Zheng, H., Chen, J., Li, S., Huang, J., and Zhou, C. (2016). Chitosanchitin nanocrystal composite scaffolds for tissue engineering. Carbohydr. Polym. 152: 832-840.

Liu, R., Xing, L., Fu, Q., Zhou, G.H., and Zhang, W.G. (2016). A review of antioxidant peptides derived from meat muscle and by-products. Antioxidants 5(3): 32

Lopez, M., Arce, L., Garrido, J., Rio, S., and Vakarcel, M. (2004). Selective extraction of astaxnthin from crustaceans by use of supercritical carbon dioxide. Talanta. 64: 726-731.

Másson, M., Holappa, J., Hjálmarsdóttir, M., Rúnarsson, Ö.V., Nevalainen, T., and Järvinen, T. (2008). Antimicrobial activity of piperazine derivatives of chitosan. Carbohydr. Polym. 74: 566-571.

Mack, D., Huntington, T., Curr, C., and Joensen, J. (2004). Evaluation of fish waste management techniques. Report to the Scottish Environment Protection Agency. Poseidon Aquatic Resource Management Ltd, Lymington, Hampshire.

Mad-Ali, S., Benjakul, S., Prodpran, T., and Maqsood, S. (2016). Charac teristics and gel properties of gelatin from goat skin as affected by pretreatments using sodium sulfate and hydrogen peroxide. J. Sci. Food Agric. 96(6): 2193-2203.

Maeda, Y., Jayakumar, R., Nagahama, H., Furuike, T., and Tamura, H (2008). Synthesis, characterization and bioactivity studies of novel $\beta$-chitin scaffolds for tissue-engineering applications. Int. J. Biol. Macromol. 42: 463-467.

Maezake, Y., Tsuji, K., and Nakagawa, Y. (1993). Hypocholesterolemic effect of chitosan in adult males. Biosci. Biotech. Biochem. 57: 1439-1444.

Majeti, N.V., and Kumar, R. (2000). A review of chitin and chitosan applications. React. Funct. Polym. 46: 1-27.

Malafaya, P.B., and Reis, R.L. (2009). Bilayered chitosan-based scaffolds for osteochondral tissue engineering: Influence of hydroxyapatite on in vitro cytotoxicity and dynamic bioactivity studies in a specific doublechamber bioreactor. Acta Biomaterialia. 5: 644-660.

Malde, M.K., Bügel, S., Kristensen, M., Malde, K., Graff, I.E., and Pedersen, J.I. (2010). Calcium from salmon and cod bone is well absorbed in young healthy men: a double-blinded randomised crossover design. Nutr. Metab. 7(1): 61

Mandeville, S., Yaylayan, V., Simpson, B.K., and Ramaswamy, H. (1991). Isolation and purification of carotenoid pigments, lipids and flavou active components from raw commercial shrimp waste. Food Biotechnol. 5: 185-195.

Manikandan, A., Thirupathi Kumara Raja, S., Thiruselvi, T., and Gnanamani, A. (2018). Engineered fish scale gelatin: An alternative and suitable biomaterial for tissue engineering. J. Bioact. Compat. Polym. 33(3): 332-346

Manivasagan, P., Bharathiraja, S., Bui, N.Q., Lim, I.G., and Oh, J. (2016) Paclitaxel-loaded chitosan oligosaccharide-stabilized gold nanoparticles as novel agents for drug delivery and photoacoustic imaging of cancer cells. Int. J. Pharm. 511(1): 367-379.

Mankin, H.J., Brandt, K.D., and Shulman, L.E. (1986). Workshop on etiopathogenesis of osteoarthritis: Proceedings and recommendations. J. Rheumatol. 13: 1130-1160.

Mann, N.J., O'Connell, S.L., Baldwin, K.M., Singh, I., and Meyer, B.J. (2010) Effects of seal oil and tuna-fish oil on platelet parameters and plasma lipid levels in healthy subjects. Lipids 45(8): 669-681.

Manninen, A.H. (2004). Protein hydrolysates in sports and exercise: a brief review. J. Sports Sci. Med. 3(2): 60

Mano, J.F., and Reis, R.L. (2007). Osteochondral defects: present situation and tissue engineering approaches. J. Tissue Eng. Regen. Med. 1 261-273.

Martın-Sanchez, A.M., Navarro, C., Pereź-Alvarez, J.A., and Kuri, V. (2009). Alternatives for Efficient and Sustainable Production of Surimi: A Review. Comp. Rev. Food Sci. Food Safety. 8: 359-374.

Martínez-Alvarez, O., Chamorro, S., and Brenes, A. (2015). Protein hydrolysates from animal processing by-products as a source of bioactive molecules with interest in animal feeding: A review. Food Res. Int 73: 204-212.

Martins, N., and Ferreira, I.C. (2017). Wastes and by-products: Upcoming sources of carotenoids for biotechnological purposes and healthrelated applications. Trends Food Sci. Technol. 62: 33-48.

Martorana, A., Gentili, A., Marzocca, A., and Rodríguez, M.S. (2002). Antimicrobial Activity of Chitosan and N-Acetylated Chitosan against Salmonella typhimurium. Chitosan in Pharmacy and Chemistry. In: Muzarelli, R.A.A., and Muzarelli, C. (Ed.). Atec Edizioni, Grottamare, pp. $183-187$

Masatoshi, M., and Junji, S. (1999). Method for simultaneously producing 
astaxanthin and chitosan from shell waste. Japanese Patent No. JP $11049972 \mathrm{~A} 2$.

Masotti, A., and Ortaggi, G. (2009). Chitosan Micro- and Nanospheres: Fabrication and Applications for Drug and DNA Delivery. Mini Rev. Med. Chem. 9: 463-469.

Matmaroh, K., Benjakul, S., Prodpran, T., Encarnacion, A.B., and Kishimura, $H$. (2011). Characteristics of acid soluble collagen and pepsin soluble collagen from scale of spotted golden goatfish (Parupeneus heptacanthus). Food Chem. 129(3): 1179-1186.

Matsumiya, M. (2007). Enzymatic production of N-acetyl-D-glucosamine using crude enzyme from the liver of squids. Food Sci. Technol. Res. 10(3): 296-299.

Matsumoto, Y., Saucedo-Castaneda, G., Revah, S., and Shirai, K. (2004). Production of $\mathrm{B}-\mathrm{N}$-acetylhexosaminidase of Verticillium lecanii by solid state and submerged fermentations utilizing shrimp waste silage as substrate and inducer. Process Biochem. 39: 665-671.

Matsuno, T., and Hirao, S. (1989). Marine carotenoids. Marine Biogenic Lipids, Fats, and Oils. In: Ackman, R.G. (Ed.). CRC Press, Boca Raton, FL, pp. 251-388.

McGinnis, L., and Wood, M. (2007). Finding new uses for fish byproducts. Agric. Res. 55: 18-19.

McWilliams, A. 2018 The Global Market for Carotenoids, 2018: https:// cdn2.hubspot.net/hubfs/308401/FOD\%20Report\%200verviews/ FOD025F\%20Report\%200verview.pdf?t=1537182142888andutm campaign=FOD025Fandutm_source=hs_automationandutm_ medium =emailandutm content $=62915556$ and hsenc $=$ p2ANqtzsxnVnBG4OCFaqzIFSA2hWloKao6uU-nSFmkcTysIhV3IRRxUQ1LqMoUZf2q_AQSvrjHQoiXQIIKRQhQeISjDWhYdEQQand_hsmi=629155 56.

Mendis, E., Rajapakse, N., Byun, H.G., and Kim, S.K. (2005). Investigation of jumbo squid (Disidicus gigas) skin gelatine peptides for their antioxidant effects. Life Sci. 77: 2166-2178.

Metusalach, Synowiecki, J., Brown, J., and Shahidi, F. (1996). Deposition and metabolism of dietary canthaxanthin in different organs of Arctic charr (Salvelinus alpinus). Aquacul. 142: 99-106.

Meyers, S.P. (1977). Using crustacean meals and carotenoid fortified diets. Feedstuffs. 43: 26-28.

Meyers, S.P., and Bligh, D. (1981). Characterization of astaxanthin pigment from heat processed crawfish waste. J. Agric. Food Chem. 29: 505-508.

Mi, F.L., Tan, Y.C., Liang, H.F., and Sung, H.W. (2002). In vivo biocompatibility and degradability of a novel injectable-chitosan-based implant. Biomaterials 23(1): 181-191.

Miki, W., Yamaguchi, K., and Konosu, S. (1982). Comparison of carotenoids in the ovaries of marine fish and shellfish. Comp. Biochem. Physiol. 71B: 7-11.

Miles, E.A., and Calder, P.C. (1998). Modulation of immune function by dietary fatty acids. Proc. Nutr. Soc. 57: 277-292.

Mitmesser, S.H., and Jensen, C.L. (2007). Roles of long-chain polyunsaturated fatty acids in the term infant: developmental benefits. Neonatal Netw. 26: 229-34.

Mizuta, S., Isobe, S., and Yoshinaka, R. (2002a). Existence of two molecular species of collagen in the muscle layer of the ascidian (Halocynthia roretzi). Food Chem. 79: 9-13.

Mizuta, S., Miyagi, T., Nishimiya, T., and Yoshinaka, R. (2002b). Partial characterization of collagen in mantle and adductor of pearl oyster (Pinctada fucata). Food Chem. 79: 319-325.

Moison, R.M.W., and Henegouwen, G.M.J.B.V. (2001). Dietary Eicosapentaenoic Acid Prevents Systemic Immunosuppression in Mice Induced by UVB Radiation. Radiation Res. 156: 36-44.

Mojarrad, J.S., Nemati, M., Valizadeh, H., Ansarin, M., and Bourbour, S. (2007). Preparation of Glucosamine from Exoskeleton of Shrimp and Predicting Production Yield by Response Surface Methodology. J. Agric. Food Chem. 55: 2246-2250.

Montana, M., Verhaeghe, P., Ducros, C., Terme, T., Vanelle, P., and Rathelot, P. (2010). Safety review: squalene and thimerosal in vaccines. Therapie 65(6): 533-541.

Montero, P., and Gómez-Guillén, M.C. (2000). Extracting conditions for megrim (Lepidorhombus boscii) skin collagen affect functional properties of the resulting gelatine. J. Food Sci. 65: 434-438.

Morales-Medina, R., Tamm, F., Guadix, A.M., Guadix, E.M., and Drusch,
S. (2016). Functional and antioxidant properties of hydrolysates of sardine (S. pilchardus) and horse mackerel (T. mediterraneus) for the microencapsulation of fish oil by spray-drying. Food Chem. 194: 1208-1216.

Morreale, P., and Manopulo, R. (1996). Comparison of the anti-inflammatory efficacy of chondroitin sulfate and diclofenac sodium in patients with knee osteoarthritis. J. Rheumatol. 23: 1385-1391.

Morrissey, M.T., and Okada, T. (2007). Marine enzymes from seafood byproducts. Maximizing the value of marine by-products. In: Shahidi, F. (Ed.). CRC Press, Boca Raton, FL, pp. 374-396.

Mourya, V.K., and Inamdar, N.N. (2008). Chitosan-modifications and applications: Opportunities galore. React. Funct. Polym. 68: 1013-1051.

Mucopolysaccharide. (2008). The Columbia Encyclopedia, Sixth Edition. 8 Nov. 2009, http://www.encyclopedia.com.

Mukundan, M.K., Gopakumar, K., and Nair, M.R. (1985). Purification of a lipase from the hepatopancreas of oil sardine (Sardinella longiceps Linnaeus) and its characteristics and properties. J. Sci. Food Agric. 36: 191-203.

Muyonga, J.H., Cole, C.G.B., and Duodu, K.G. (2004). Extraction and physico-chemical characterisation of Nile perch (Lates niloticus) skin and bone gelatine. Food Hydrocolloid. 18: 581-592.

Muzzarelli, R.A.A. (1977). Chitin. Pergamon Press, Oxford. UK, pp. 11-14.

Muzzarelli, R.A.A., Frega, N., Miliani, M., Muzzarelli, C., and Cartolari, M. (2000). Interactions of chitin, chitosan, N-lauryl chitosan and Ndimethylaminopropyl chitosan with olive oil. Carbohydr. Polym. 43: 263-268.

Myrnes, B., and Johansen, A. (1994). Recovery of lysozyme from scallop waste. Prep Biochem 24: 69-80.

Nagai, T., Ogawa, T., Nakamura, T., Ito, T., Nakagawa, H., Fujiki, K., Nakao, M., and Yano, T. (1999). Collagen of edible jellyfish exumbrella. J. Sci. Food Agric. 79: 855-858.

Nagai, T., and Suzuki, N. (2000). Isolation of collagen from fish waste material-skin, bone and fins. Food Chem. 68: 277-281.

Najafian, L., and Babji, A.S. (2012). A review of fish-derived antioxidant and antimicrobial peptides: their production, assessment, and applications. Peptides 33(1): 178-185

Nakano, T., Sunwoo, H.H., Li, X., Price, M.A., and Jeong, S.S. (1996). Study of sulfated glycosaminoglycans from porcine skeletal muscle epimysium including analysis of iduronosyl and glucoronosyl residues in galactosaminoglycan fractions. J. Agric. Food Chem. 44: 1424-1434.

Nam, K.S., Kim, M.K., and Shon, Y.H. (2007). Chemopreventive effect of chitosan oligosaccharide against colon carcinogenesis. J. Microbiol. Biotechnol. 17(9): 1546-1549.

Nasri, R., Younes, I., Jridi, M., Trigui, M., Bougatef, A., Nedjar-Arroume, N., Dhulster, P., Nasri, M., and Karra-Châabouni, M. (2013). ACE inhibitory and antioxidative activities of Goby (Zosterissessor ophiocephalus) fish protein hydrolysates: effect on meat lipid oxidation. Food Res. Int. 54(1): 552-561.

Nayak, J., Nair, P.G.V., Ammu, K., and Mathew, S. (2003). Lipase activity in different tissues of four species of fish: Rohu (Labeo rohita Hamilton): oil sardine (Sardinella longiceps Linnaeus): mullet (Liza subviridis Valenciennes) and Indian mackerel (Rastrelliger kanagurta Cuvier). J. Sci. Food Agric. 83: 1139-1142.

Naziri, E., Mitić, M.N., and Tsimidou, M.Z. (2016). Contribution of tocopherols and squalene to the oxidative stability of cold-pressed pumkin seed oil (Cucurbita pepo L.). Eur. J. Lipid Sci. Technol. 118(6): 898905.

Nelis, H.J., Lavens, P., Moens, L., Sorgeloos, P., and De Leenheer, A.P. (1989). Carotenoids in relation to Artemia development. Biochemistry and Cell Biologyof Artemia. In: MacRae, T.H., Bagslaw, J.C., and Warner, A.H. (Ed.). CRC Press, Boca Raton, FL, pp. 159-190.

Nemati, M., Kamilah, H., Huda, N., and Ariffin, F. (2016). In vitro calcium availability in bakery products fortified with tuna bone powder as a natural calcium source. Int. J. Food Sci. Nutr. 67(5): 535-540.

Nergiz, C., and Çelikkale, D. (2011). The effect of consecutive steps of refining on squalene content of vegetable oils. J. Food Sci. Technol. 48(3): 382-385.

Neumann, A., Scholz-Muramatsu, H., and Diekert, G. (1994). Tetrachloroethene metabolism of Dehalospirillum multivorans. Arch. Microbiol. 162: 295-301.

Newmark, H.L. (1999). Squalene, olive oil, and cancer risk: Review and hy- 
pothesis. Ann. N. Y. Acad. Sci. 889: 193-203.

Newton, I.S. (1996). Long chain fatty acids in health and nutrition. J. Food Lipids 3(4): 233-249.

Nichols, P.D., Mooney, B., Virtue, P., and Elliott, N. (1998). Nutritional value of Australian Fish: oil, fatty acid and cholesterol composition of edible species. FRDC Project, 95/122, Australian Fisheries and Development Corporation.

Ninan, G., Zynudheen, A.A., and Joseph, J. (2011). Physico-chemical and textural properties of gelatins and water gel desserts prepared from the skin of freshwater carps. Fish. Technol. 48(1):

Nishimura, K., Nishimura, S., Nishi, N., Saiki, I., Tokura, S., and Azuma, I. (1984). Immunolgical activity of Chitin and its derivatives. Vaccine. 2: 93-99.

Nishmoto, S.K., Waite, J.H., Nishimoto, M., and Kriwacki, R.W. (2003) Structure, activity, and distribution of fish osteocalcin. J. Biol. Chem. 278: 11843-11848.

No, H.K., and Meyers, S.O. (1997). Chitin Composition. Chitin Handbook. In: Muzzarelli, R.A.A., and Peter, M.G. (Ed.). European Chitin Society, Grottammare, pp. 475-489.

No, H.K., Park, N.Y., Lee, S.H., and Meyers, S.P. (2002). Antibacterial activity of chitosans and chitosan oligomers with different molecular weights. Int. J. Food Microbiol. 74: 65-72.

Nomura, Y. (2004). Properties and utiliztion of shark collagen. Developments in Food Science. Elsevier, 42: pp. 147-158.

Nomura, Y., Sakai, H., Ishii, Y., and Shirai, K. (1996). Preparation and some properties of Type I collagen from fish scales. Biosci. Biotechnol. Biochem. 60: 2092-2094.

Nomura, Y., Toki, S., Ishii, Y., and Shirai, K. (2001). Effect of transglutaminase on reconstruction and physicochemical properties of collagen gel from shark type I collagen. Biomacromolecules 2(1): 105-110.

Nomura, Y., Yamano, M., and Shirai, K. (1995). Renaturation of $\alpha 1$ of collagen type I from shark skin. J. Food Sci. 60: 1233-1236.

Norland, R.E. (1990). Fish gelatine. Advances in fisheries technology and biotechnology for increased profitability. In: Voight, M.N., and Botta, J.K. (Ed.). Technomic Publishing Co, Lancaster, pp. 325-333.

Novoa-Carballal, R., Pérez-Martín, R., Blanco, M., Sotelo, C.G., Fassini, D., Nunes, C., Coimbra, M.A., Silva, T.H., Reis, R.L., and Vázquez, J.A (2017). By-products of Scyliorhinus canicula, Prionace glauca and Raja clavata: A valuable source of predominantly 6 S sulfated chondroitin sulfate. Carbohydr. Polym. 157: 31-37.

Ockerman. (1992). Fishery by-products. Fish processing technology. In: Hall, G.M. (Ed.). Academic and Professional, New York, USA, pp. 155-192.

Ohkawa, K., Takahashi, Y., and Yamamoto, H. (1999). Self-assembling capsule and fiber formations of polyion complexes of chitosan and poly $(\alpha$-L-glutamic acid). Macromol. Rapid. Commun. 21: 223-225.

Ohshima, T. (1998). Recovery and use of nutraceutical products from marine resources. Food Technol. 52: 50-52.

Okada, T., and Morrissey, M.T. (2007). Production of n-3 Polyunsaturated Fatty Acids: Concentrate from Sardine Oil by Lipase-Catalyzed Hydrolysis. Food Chem. 103(4): 1411-1419.

Olsen, R.L., Johanson, A., and Myrnes, B. (1990). Recovery of enzymes from shrimp wastes. Process Biochem. 25: 67-68.

Olsen, R.L., Toppe, J., and Karunasagar, I. (2014). Challenges and realistic opportunities in the use of by-products from processing of fish and shellfish. Trends Food Sci. Technol. 36(2): 144-151.

Osterud, B., Elvevoll, E., Barstad, H., Brox, J., Halvorsen, H., Lia, K., Olsen, J.O., Olsen, R.L., Sissener, C., Rekdal, O., and Vognild, E. (1995). Effect of marine oils supplementation on coagulation and cellular activation in whole blood. Lipids 30(12): 1111-1118.

Ozawa, M., and Suguru, S. (2002). Microstructural development of natural hydroxyapatite originated from fish-bone waste through heat treatment. J. Am. Ceram. Soc. 85: 1315-1317.

Paños, I., Acosta, N., and Heras, A. (2008). New drug delivery systems based on chitosan. Curr. Drug Discov. Technol. 5: 333-341.

Pal, A., Paul, S., Choudhury, A.R., Balla, V.K., Das, M., and Sinha, A. (2017). Synthesis of hydroxyapatite from Lates calcarifer fish bone for biomedical applications. Mater. Lett. 203: 89-92.

Pallela, R., Venkatesan, J., Janapala, V.R., and Kim, S.K. (2012). Biophysicochemical evaluation of chitosanhydroxyapatite-marine sponge collagen composite for bone tissue engineering. Biomed. Mater. Res.
100A: 486-495

Pangon, A., Saesoo, S., Saengkrit, N., Ruktanonchai, U., and Intasanta, V. (2016). Hydroxyapatite-hybridized chitosan/chitin whisker bionanocomposite fibers for bone tissue engineering applications. Carbohydr. Polym. 144: 419-427.

Papineau, A.M., Hoover, D.G., Knorr, D., and Farkas, D.F. (1991). Antimicrobial effect of water-soluble chitosans with high hydrostatic pressure. Food Biotechnol. 5: 45-57.

Parani, S., Pandian, K., and Oluwafemi, O.S. (2018). Gelatin stabilization of quantum dots for improved stability and biocompatibility. Int. J. Biol. Macromol. 107: 635-641.

Park, H.J., Joon, B.Y., Bae, H.J., Kim, Y.T., and Cha, D.S. (2007). Film-forming composition for hard capsules comprising fish gelatine and its preparation method. Wipo Patent WO/2007/123350.

Park, J.K., Chung, M.J., Choi, H.N., and Park, Y.I. (2011). Effects of the molecular weight and the degree of deacetylation of chitosan oligosaccharides on antitumor activity. Int. J. Mol. Sci. 12(1): 266-277.

Park, P.J., Je, J.Y., Bgun, H.G., Moon, S.H., and Ku, S.K. (2004). Antimicrobial activity of hetero-chitosans and their oligosaccharides with different molecular weight. J. Microbiol. Biotechnol. 14: 317-323.

Park, P.J., Je, J.Y., Jung, W.K., Ahn, C.B., and Kim, S.K. (2004). Anticoagulant activity of heterochitosans and their oligosaccharide sulphates. Eur. Food Res. Technol. 219: 529-533.

Park, P.J., Lee, S.H., Byun, H.G., Kim, S.H., and Kim, S.K. (2002). Purification and characterization of a collagenase from the mackerel, Scombe japonicus. J. Biochem. Mol. Biol. 35(6): 576-582.

Paszkiewicz, M., Gołąbiewska, A., Rajski, Ł., Kowal, E., Sajdak, A., and Zaleska-Medynska, A. (2016). The antibacterial and antifungal textile properties functionalized by bimetallic nanoparticles of $\mathrm{Ag} / \mathrm{Cu}$ with different structures. J. Nanomater. (3): 1-13.

Patton, J.S. (1975). High levels of pancreatic nonspecific lipase in rattlesnake and leopard shark. Lipids 10: 562-564.

Patton, J.S., Nevenzel, J.C., and Benson, A.A. (1975). Specificity of digestive lipases in hydrolysis of wax esters and triglycerides studied in anchovy and other selected fish. Lipids 10: 575-583.

Pawar, S.V., and Yadav, G.D. (2014). PVA/chitosan-glutaraldehyde crosslinked nitrile hydratase as reusable biocatalyst for conversion of nitriles to amides. J. Mol. Catal. B: Enzym. 101: 115-121.

Pawelec, K.M., Best, S.M., and Cameron, R.E. (2016). Collagen: A network for regenerative medicine. J. Mater. Chem. B 4: 6484-6496.

Peinado, I., Koutsidis, G., and Ames, J. (2016). Production of seafood flavour formulations from enzymatic hydrolysates of fish by-products LWT-Food Sci. Technol. 66: 444-452.

Peter, M., Sudheesh Kumar, P.T., Binulal, N.S., Nair, S.V., Tamura, H., and Jayakumar, R. (2009). Development of novel $\alpha$-chitin/nanobioactive glass ceramic composite scaffolds for tissue engineering applications. Carbohydr. Polym. 78: 926-93.

Petit-Omega-dsm.com. Effects of Fatty Acids on Reproduction in the Dairy Cow: The Good and the Bad Dairy and Swine Research and Development Centre Agriculture and Agri-Food Canada.

Peyronel, D., Artaud, J., latrides, M.C., Rancurel, P., and Chevalier, J.L. (1984). Fatty acid and squalene compositions of Mediterranean Centrophorus spp. egg and liver oils in relation to age. Lipids. 19: 634-48.

Phleger, C.F., Nichols, P.D., and Virtue, P. (1998). Lipids and trophodynamics of Antarctic zooplankton. Comp. Biochem. Physiol. B: Biochem. Mol. Biol. 120(2): 311-323.

Picot, L., Ravallec, R., Fouchereau-Péron, M., Vandanjon, L., Jaouen, P., Chaplain-Derouiniot, M., Guérard, F., Chabeaud, A., LeGal, Y., Alvarez, O.M., Bergé, J., Piot, J., Batista, I., Pires, C., Thorkelsson, G., Delannoy, C., Jakobsen, G., Johansson, I., and Bourseau, P. (2010). Impact of ultrafiltration and nanofiltration of an industrial fish protein hydro lysate on its bioactive properties. J. Sci. Food Agric. 90: 1819-1826.

Pipitone, V.R. (1991). Chondroprotection with chondroitin sulfate. Drugs Exp. Clin. Res. 17: 3-7.

Pokkanta, P., Sookwong, P., Tanang, M., Setchaiyan, S., Boontakham, P., and Mahatheeranont, S. (2019). Simultaneous determination of tocols, $\mathrm{y}$-oryzanols, phytosterols, squalene, cholecalciferol and phylloquinone in rice bran and vegetable oil samples. Food Chem. 271: 630-638.

Porfírio, E., and Fanaro, G.B. (2016). Collagen supplementation as a complementary therapy for the prevention and treatment of osteoporo- 
sis and osteoarthritis: A systematic review. Rev. Bras. Geriatr. Gerontol. 19: 153-164.

Power, O., Hallihan, A., and Jakeman, P. (2009). Human insulinotropic response to oral ingestion of native and hydrolysed whey protein. Amino Acids. 37: 333-339.

Protan Laboratories, Inc. (1987). Sea CureTM Chitosan for Immobilization of Enzymes, PL1-003, Redmond, WA.

Prabaharan, M. (2008). Chitosan derivatives as promising materials for controlled drug delivery. J. Biomater. Appl. 23(1): 5-36.

Prabaharan, M., and Mano, J.F. (2007). Synthesis and Characterization of Chitosan-graft-Poly (3-(trimethoxysilyl) propyl methacrylate) Initiated by Ceric (IV) Ion. J. Macromol. Sci., Part A 44(5): 489-494.

Prabaharan, M., Rodriguez-Perez, M.A., de Saja, J.A., and Mano, J.F. (2007). Preparation and Characterization of Poly(L-lactic acid)-Chitosan Hybrid Scaffolds with Drug Release Capability. J. Biomed. Mater. Res., Part B: Appl. Biomat. 81B: 427-434.

Prakash, I., and Dubois, G.E. U.S. Patent No. 8,956,677. Washington, DC: U.S. Patent and Trademark Office.

Prashanth, K.V.H., and Tharanathan, R.N. (2007). Chitin/chitosan: modifications and their unlimited application potential-an overview. Trends Food Sci. Technol. 18: 117-131.

Preuss, H.G., and Kaats, G.R. (2006). Chitosan as a Dietary Supplement for Weight Loss: A Review. Curr.Nutr. Food Sci. 2: 297-311.

Puppel, K., Kuczyńska, B., Nałęcz-Tarwacka, T., Gołębiewski, M., Sakowski, T., Kapusta, A., and and Balcerak, M. (2016). Effect of supplementation of cows diet with linseed and fish oil and different variants of $\beta$-lactoglobulin on fatty acid composition and antioxidant capacity of milk. J. Sci. Food Agric. 96(6): 2240-2248.

Putnam, J.C., Carlson, S.E., DeVoe, P.W., and Barness, L.A. (1982). The effect of variations in dietary fatty acids on the fatty acid composition of erythrocyte phosphatidylcholine and phosphatidylethanolamine in human infants. Am. J. Clin. Nutr. 36: 106-114.

Qin, Y., Zhang, S., Yu, J., Yang, J., Xiong, L., and Sun, Q. (2016). Effects of chitin nano-whiskers on the antibacterial and physicochemical properties of maize starch films. Carbohydr. Polym. 147: 372-378.

Raa, J., and Walther, B.T. (1989). Purification and characterization of chymotrypsin, trypsin and elastase like proteinases from cod (Gadus morhua L.). Comp. Biochem. Physiol. 93B: 317-324.

Raafat, D., von Bargen, K., Haas, A., and Sahl, H. (2008). Insight into the mode of action of Chitosan as an Antibacterial Compound. Appl. Environ. Microbiol. 74: 3764-3773.

Raghunath, M.R., and Gopakumar, K. (2002). Trends in production and utilization of fish silage. J. Food Sci. Technol. 39: 103-110.

Rahman, M.A. (2019). Collagen of Extracellular Matrix from Marine Invertebrates and Its Medical Applications. Mar. Drugs 17(2): 118.

Rai, A.K., Jini, R., Swapna, H.C., Sachindra, N.M., Bhaskar, N., and Baskaran, V. (2011). Application of native lactic acid bacteria (LAB) for fermentative recovery of lipids and proteins from fish processing wastes: Bioactivities of fermentation products. J. Aquat. Food Prod. Technol. 20(1): 32-44.

Rajapakse, N., Jung, W.K., Mendis, E., Moon, S.H., and Kim, S.K. (2005). A novel anticoagulant purified from fish protein hydrolysate inhibits factor XIla and platelet aggregation. Life Sci. 76: 2607-2619.

Rajasekaran, A., and Kalaivani, M. (2013). Designer foods and their benefits: A review. J. Food Sci. Technol. 50(1): 1-16.

Ramírez, A. (2013). Innovative uses of fisheries by-products. GLOBEFISH Research Programme 110: I.

Ramakrishnan, C., and Prasad, N. (1972). Rigid-body refinement and conformation of $\alpha$-chitin. Biochem. Biophys. Acta 261: 123-135.

Rao, C.V., Newmark, H.L., and Reddy, B.S. (1998). Chemopreventive effect of squalene on colon cancer. Carcinogenesis. 19: 287-290.

Rasco, B.A., and Hultin, H.O. (1988). A comparison of dogfish and porcine pancreatic lipases. Comp. Biochem. Physiol., Part B: Biochem. Mol. Biol. 89: 671-677.

Rasmussen, R.S., and Morrissey, M.T. (2007). Marine Biotechnology for Production of Food Ingredients. Adv. Food and Nutr. Res. 52: 237292.

Rasti, B., Erfanian, A., and Selamat, J. (2017). Novel nanoliposomal encapsulated omega-3 fatty acids and their applications in food. Food Chem. 230: 690-696.

Ratnayake, W.M.N., Olisson, B., Matthews, D., and Ackman, R.G. (1988).
Preparation of Omega-3 PUFA Concentrates from Fish Oils via Urea Complexation. Fat Sci. Technol. 90: 381-386.

Reddy, L.H., and Couvreur, P. (2009). Squalene: A natural triterpene for use in disease management and therapy. Adv. Drug Delivery Rev. 61(15): 1412-1426.

Reece, P. (1988). Recovery of proteases from fish waste. Process Biochem. 23(6): 62-66.

Regenstein, J.M., Chaudry, M.M., and Regenstein, C.E. (2003). The kosher and halal food laws. Compr. Rev. Food Sci. Food Saf. 2(3): 111-127.

Regenstein, J.M., and Zhou, P. (2007). Collagen and gelatine from marine by-products. Maximizing the value of marine by-products. In: Shahidi, F. (Ed.). CRC Press, Boca Raton, FL, pp. 279-303.

Reginster, J.Y.L., Bruyere, O., and Cooper, C. (2018). Different glucosamine sulfate products generate different outcomes on osteoarthritis symptoms. Ann. Rheum. Dis. 77(7): e39-e39.

Remunan-Lopez, C., Portero, A., Vila-Jato, J.L., and Alonso, M.J. (1998). Design and evaluation of chitosan/ethylcellulose mucoadhesive bilayered devices for buccal drug delivery. J. Contr. Release. 55: 143-152.

Revathi, M., Saravanan, R., and Shanmugam, A. (2012). Production and characterization of chitinase from Vibrio species, a head waste of shrimp Metapenaeus dobsonii (Miers, 1878) and chitin of Sepiella inermis Orbigny, 1848. Adv. Biosci. Biotechnol. 3(04): 392.

Rinaudo, M. (2007). Main properties and current applications of some polysaccharides as biomaterials. Polym. Int. 57: 397-430.

Ritchie, A.H., and Mackie, I.M. (1982). Preparation of fish protein hydrolysates. Anim. Feed Sci. Technol. 7: 125-133.

Robledo, N., Vera, P., López, L., Yazdani-Pedram, M., Tapia, C., and Abugoch, L. (2018). Thymol nanoemulsions incorporated in quinoa protein/chitosan edible films; antifungal effect in cherry tomatoes. Food Chem. 246: 211-219.

Roller, S., and Covill, N. (1999). The antifungal properties of chitosan in laboratory media and apple juice. Int. J. Food Microbiol. 47: 67-77.

Ronca, F., and Palmieri, L. (1998). Anti-inflammatory activity of chondroitin sulfate. Osteoarthr. Cartilage 6: 14-21.

Roy, P. (1998). Enzymatic hydrolysis of marine raw materials for production of cosmetic ingredients. COSM'ING 98.

Rustad, T. (2003). Utilisation of marine by-products. Electron. J. Environ. Agric. Food Chem. 2: 458-463.

Rustad, T., Storr $\varnothing$, I., and Slizyte, R. (2011). Possibilities for the utilisation of marine by-products. Int. J. Food Sci. Technol. 46(10): 2001-2014.

Sachindra, N.M., Bhaskar, N., and Mahendrakar, N.S. (2005b). Carotenoids in crabs from marine and fresh waters of India. Lebens Wiss undTechnol. 38: 221-225.

Sachindra, N.M., Bhaskar, N., and Mahendrakar, N.S. (2005). Carotenoids in different body components of Indian shrimps. J. Sci. Food Agri. 85: 167-172.

Sachindra, N.M., Bhaskar, N., and Mahendrakar, N.S. (2006). Recovery of carotenoids from shrimp waste in organic solvents. Waste Mgt. 26: 1092-1098.

Sachindra, N.M., Bhaskar, N., Siddegowda, G.S., Sathisha, A.D., and Suresh, P.V. (2007). Recovery of carotenoids from ensilaged shrimp waste. Bioresour. Technol. 98: 1642-1646.

Sachindra, N.M., and Mahendrakar, N.S. (2005). Process optimization for extraction of carotenoids from shrimp waste with vegetable oils. $\mathrm{Bi}$ oresour. Technol. 96: 1195-1200.

Sae-Leaw, T., and Benjakul, S. (2018). Lipase from liver of seabass (Lates calcarifer): Characteristics and the use for defatting of fish skin. Food Chem. 240: 9-15.

Sahraee, S., Milani, J.M., Ghanbarzadeh, B., and Hamishehkar, H. (2017). Physicochemical and antifungal properties of bio-nanocomposite film based on gelatin-chitin nanoparticles. Int. J. Biol. Macromol. 97: 373-381.

Saito, H., Kotani, Y., Keriko, J.M., Xue, C., Taki, K., Ishihara, K., Ueda, T., and Miyata, S. (2002). High levels of n-3 polyunsaturated fatty acids in Euphausia pacifica and its role as a source of docosahexaenoic and icosapentaenoic acids for higher trophic levels. Mar. Chem. 78: 9-28.

Sakai, K., Nanjo, F., and Usui, T. (1990). Production and utilization of oligosaccharides from chitin and chitosan. Denpun Kagaku. 37: 79-86.

Sanchez-Chiang, L., and Ponce, O. (1981). Gastric sinogens and gastrisins from Merluccaius gayi-purification and properties. Comp. Biochem. Physiol. 68B: 251-257. 
Sanford, P.A. (1989). Chitosan: commercial uses and potential applications. Chitin and chitosan. pp. 51-59.

Sarabia, A.I., Gómez-Guillén, M.C., and Montero, P. (2000). The effect of added salts on the viscoelastic properties of fish skin gelatine. Food Chem. 70: 71-76.

Sargent, J., Bell, G., Mcevoy, L., Tocher, D., and Estevez, A. (1999). Recent developments in essential fatty acid nutrition of fish. J. Aquacul. 177: 91-199.

Sargent, J., Henderson, R.J., and Tocher, D.R. (1989). The lipids. Fish Nutrition. In: Halver, J.E. (Ed.). Academic Press, San Diego, pp. 153-218.

Sarmadi, B.H., and Ismail, A. (2010). Antioxidative peptides from food proteins: a review. Peptides 31(10): 1949-1956.

Sarode, S., Upadhyay, P., Khosa, M.A., Mak, T., Shakir, A., Song, S., and Ullah, A. (2019). Overview of wastewater treatment methods with special focus on biopolymer chitin-chitosan. Int. J. Biol. Macromol. 121: $1086-1100$.

Sashai, A.S., and Manocha, M.S. (1993). Chitinases of fungi and plants: their involvement in morphogenesis and host-parasite interaction. FEMS Microbiol. Rev. 11: 317-338.

Sathivel, S., Prinyawiwatkul, W., King, J.M., Grimm, C.C., and Lloyd, S. (2003). Oil production from catfish viscera. J. Am. Oil Chem. Soc. 80 $377-382$.

Schacky, C.V. (2000). n-3 fatty acids and the prevention of coronary atherosclerosis. Am. J. Clin. Nutr. 71: 224-227.

Schiedt, K., Vecchi, M., and Glinz, E. (1986). Astaxanthin and its metabolites in wild raibow trout (Salmo gairdneri R.). Comp. Biochem. Physiol. 83B: 9-12.

Schiller, R.N., Barrager, E., Schauss, A.G., and Nichols, E.J. (2001). A randomized, double-blind, placebo-controlled study examining the effects of a rapidly soluble chitosan dietary supplment on weight loss and body composition in overweight and mildly obese individuals. J. A. N. A. 4: 34-41.

Schlenk, H., and Holman, R.T. (1950). Separation and Stabilization of Fatty Acids by Urea Complexes. J. Am. Oil Chem. Soc. 27: 5001-5004.

Schreiner, M., Hulan, H.W., Razzazi-Fazeli, E., Bohm, J., and Iben, C. (2004). Feeding laying hens seal blubber oil: effects on egg yolk incorporation, stereospecific distribution of omega-3 fatty acids, and sensory aspects. Poult. Sci. 83: 462-473.

Schrieber, R., and Gareis, H. (2007). Gelatine handbook. Wiley-VCH GmbH and Co., Weinhem.

Scolastici, C., Ong, T.P., and Moreno, F.S. (2004). Squalene does not exhibit a chemopreventive activity and increases plasma cholesterol in a Wistar rat hepatocarcinogenesis model. Nutr. Cancer 50: 101-109.

Sebti, I., Martial-Gros, A., Carnet-Pantiez, A., Grelier, S., and Coma, V. (2005). Chitosan Polymer as Bioactive Coating and Film against Aspergillus niger Contamination. J. Food Sci. 70: M100-M104.

Senaratne, L.S., Park, P., and Kim, S. (2006). Isolation and characterization of collagen from brown backed toadfish (Lagocephalus gloveri) skin. Bioresource Technol. 97: 191-197.

Senda, T., He, Y., and Inoue, Y. (2001). Biodegradable blends of poly( $\varepsilon$ caprolactone) with $\alpha$-chitin and chitosan: specific interactions, thermal properties and crystallization behaviour. Poly. Int. 51: 33-39.

Senevirathne, M., and Kim, S.-K. (2012). Utilization of Seafood Processing By-products: Medicinal Applications. Advances in Food and Nutrition Research. In: Se-Kwon, K. (Ed.). Academic Press, New York, pp. 495-512.

Serrano, M.C., Nardecchia, S., García-Rama, C., Ferrer, M.L., CollazosCastro, J.E., del Monte, F., and Gutiérrez, M.C. (2014). Chondroitin sulphate-based 3D scaffolds containing MWCNTs for nervous tissue repair. Biomaterials 35(5): 1543-1551.

Severus, W.E., Ahrens, B., and Stoll, A. (1999). Omega-3 fatty acids-the missing link? Arch. Gen, Psych. 56: 380-381.

Shahar, E., Folsom, A.R., Melnick, S.L., Tockman, M.S., Comstock, G.W., Gennaro, V., Higgins, M.W., Sorlie, P.D., Ko, W.-J., and Szklo, M. (1994). Dietary $n-3$ polyunsaturated fatty acids and smoking-related chronic obstructive pulmonary disease. N. Eng. J. Med. 331: 228-233.

Shahidi, F. (2002). Marine nutraceuticals. Inform. 13: 57-62.

Shahidi, F. (Ed.). (2006). Maximising the value of marine by-products. Woodhead Publishing.

Shahidi, F. (2007). Nutraceuticals and Healthful Products from Aquatic Resources: Feeding and healing of humans. J. Ocean. Technol. 2: 37-48.
Shahidi, F. (2012). Nutraceuticals, functional foods and dietary supplements in health and disease. J. Food Drug Anal. 20(1): 226-230.

Shahidi, F. (2007). Chitin and chitosan from marine by-products. Maximizing the value of marine by-products. In: Shahidi, F. (Ed.). CRC Press, Boca Raton, FL, pp. 340-373.

Shahidi, F. (2007). Marine oils from seafood waste. Maximizing the value of marine by-products. In: Shahidi, F. (Ed.). CRC Press, Boca Raton, FL, pp. 258-278.

Shahidi, F. (1997). Seafood safety, processing, and biotechnology: An Overview. Seafood safety, processing, and biotechnology. In: Shahidi, F., Yvonne, J., and Kitts, D.D. (Ed.). Technomic Publishing Co. Inc., Lancaster, Basel, pp. 1-4.

Shahidi, F. (1994). Proteins from seafood processing discards. Seafoods proteins. In: Sikorski, Z.E., Pan, B.S., and Shahidi, F. (Ed.). Chapman and Hall, New York, NY, pp. 171-193.

Shahidi, F. (1998). Seal fishery and product development. Canada Science Publishing Company, St. John's, p. 231.

Shahidi, F., and Ambigaipalan, P. (2015). Novel functional food ingredients from marine sources. Curr. Opin. Food Sci. 2: 123-129.

Shahidi, F., Amorowicz, R., Synowiecki, J., and Naczk, M. (1994). Extraction and Concentration of Omega-3 Fatty Acids of Seal Blubber. Developments in Food Engineering. In: Yano, T., Matsuno, R., and Nakamura, K. (Ed.). Blackie Academic and Professional, New York, NY, USA, pp. 627-629.

Shahidi, F., and Brown, J.A. (1998). Carotenoid pigments in seafoods and aquaculture. Crit. Rev. Food Chem. 38(1): 1-67.

Shahidi, F., Han, X.Q., and Synowiecki, J. (1995). Production and characteristics of protein hydrolysates from capelin (Mallotus villosus). Food Chem. 53: 285-293.

Shahidi, F., Kamil, J., Jeon, Y.J., and Kim, S-K. (2002). Antioxidant role of chitosan. In a cooked cod (Gadus Morhua) Model System. J. Food Lipids. 9: 57-64.

Shahidi, F., and Kamil, J.Y.V.A. (2001). Enzymes from fish and aquatic invertebrates and their application in the food industry. Trends Food Sci. Technol. 12: 435-464.

Shahidi, F., Kamil, J.Y.V.A., and Jeon, Y.J. (1999). Food applications of chitin and chitosans. Trends Food Sci. Technol. 10: 37-51.

Shahidi, F., and Kim, S.K. (2002). Effects of Processing and Storage. Bioactive Compounds in Foods. ACS Symposium Series 816. In: Lee, T.C. and Ho, C.T. (Ed.). American Chemical Society, Washington, DC, pp. $1-13$

Shahidi, F., and Metusalach and Brown, J.A. (1998). Carotenoid Pigments in Seafoods and Aquaculture. Crit. Rev. Food Sci. Nutr. 38: 1-67.

Shahidi, F., and Metusalach and. Brown, J.A. (1998). Carotenoid pigments in seafoods and aquaculture. Crit. Rev. Food Sci. Nutr. 38: 1-67.

Shahidi, F., and Rahman, M.J. (2018). Bioactives in seaweeds, algae, and fungi and their role in health promotion. J. Food Bioact 2: 58-81.

Shahidi, F., and Synowiecki, J. (1991). Isolation and characterization of nutrients and value-added products from snow crab (Chinoecetes opilio) and shrimp (Pandalus borealis) processing discards. J. Agric. Food Chem. 39: 1527-1532.

Shahidi, F., and Synowiecki, J. (1991). Isolation and characterization of nutrients and value added products from snow crab (Chinoecetes opilio) and shrimp (Pandalus borealis) processing discards. J. Agric Food Chem. 39: 1527-1532.

Shahidi, F., Synowiecki, J., and Balejko, J. (1994). Proteolytic hydrolysis of muscle proteins from Harp seal (Phoca groenladica). J. Agric. Food Chem. 42: 2634-2638.

Shahidi, F., Wanasundara, P.K.J.P.D., and Wanasundara, U.N. (1996). Seal Blubber Oil: A Novel Source Of $\Omega 3$ Fatty Acids. J. Food Lipids. 3: 293306.

Shahidi, F., Wanasundara, U., and Brunet, N. (1994). Oxidative stability of oil from blubber of harp seal (Phagophilus groenlandicus) as assessed by NMR and standard procedures. Food Res. Int. 27: 555-562

Shahidi, F., and Wanasundara, U.N. (1998). Omega-3 fatty acid concentrates: nutritional aspects and production technologies. Trends Food Sci. Technol. 9(6): 230-240.

Shahidi, F., and Zhong, Y. (2007). Antioxidants from marine by-products. Maximizing the value of marine by-products. In: Shahidi, F. (Ed.). CRC Press, Boca Raton, FL, pp. 397-412.

Shamsuzzaman, K., and Haard, N.F. (1983). Evaluation of harp seal gastric 
protase as a rennet substitute for cheddar cheese. J. Food Sci. 48: 179-182.

Shamsuzzaman, K., and Haard, N.F. (1984). Purification and characterization of a chymosin-like protease from gastric mucosa of harp seal (Paophilus groenlandicus). Can. J. Biochem. Cell Biol. 62: 699-708.

Shankar, S., Reddy, J.P., Rhim, J.W., and Kim, H.Y. (2015). Preparation, characterization, and antimicrobial activity of chitin nanofibrils reinforced carrageenan nanocomposite films. Carbohydr. Polym. 117: 468-475.

Sheehan, J.P., Wei, I.W., Ulchaker, M., and Tserng, K.Y. (1997). Effect of high fiber intake in fish oil-treated patients with non-insulin-dependent diabetes mellitus. Am. J. Clin. Nutr. 66: 1183-1187.

Shelma, R., Paul, W., and Sharma, C.P. (2008). Chitin nanofibre reinforced thin chitosan films for wound healing application. Trends Biomater. Artif. Organs 22(2): 111-115.

Shen, K.T., Chen, M.H., Chan, H.Y., Jeng, J.H., and Wang, Y.J. (2009). Inhibitory effects of chitooligosaccharides on tumor growth and metastasis. Food Chem. Toxicol. 47(8): 1864-1871.

Shen, X.R., Kurihara, H., and Takahashi, K. (2007). Characterization of molecular species of collagen in scallop mantle. Food Chem. 102: 1187-1191.

Shi, J., and Maguer, M.L. (2000). Lycopene in tomatoes: chemical and physical properties affected by food processing. Crit. Rev. Food Sci. Nutr. 40(1): 1-42.

Shi, P., Liu, M., Fan, F., Yu, C., Lu, W., and Du, M. (2018). Characterization of natural hydroxyapatite originated from fish bone and its biocompatibility with osteoblasts. Mater. Sci. Eng., C 90: 706-712.

Shikhman, A.R., Kuhn, K., Alaaeddine, N., and Lotz, M. (2001). N-Acetylglucosamine Prevents IL-1 $\beta$-Mediated Activation of Human Chondrocytes. J. Immunol. 166: 5155-5160.

Shimizu, Y., Arai, K., Ise, S., and Shimasaki, H. (2001). Dietary fish oil for hens affects the fatty acid composition of egg yolk phospholipids and gives a valuable food with an ideal balance of $n-6$ and $n-3$ essential fatty acids for human nutrition. J. Oleo Sci. 50: 797-803.

Shinposya, N.Y. (1989). Cosmetics and raw materials, changing with deregulations. Squalene, revalued in cosmetics. Long-term prospects for new applications. Oil and Fat. 52: 22-23.

Shotton, D.M. (1970). Methods in enzymology. In: Colowik, S.P., and Kaplan, N.O. (Ed.). Academic Press, New York, pp. 113-140.

Sila, A., and Bougatef, A. (2016). Antioxidant peptides from marine byproducts: Isolation, identification and application in food systems. A review. J. Funct. Foods 21: 10-26.

Silva, T., Moreira-Silva, J., Marques, A., Domingues, A., Bayon, Y., and Reis, R. (2014). Marine origin collagens and its potential applications. Mar. Drugs 12(12): 5881-5901.

Simopoulos, A.P. (1989). Summary of the NATO Advanced Research Workshop on Dietary $\omega 3$ and $\omega 6$ Fatty Acids: Biological Effects and Nutritional Essentiality. J. Nutr. 119: 521-528.

Simpson, B.K. (2007). Pigments from by-products of seafood processing. Maximizing the value of marine by-products. In: Shahidi, F. (Ed.). CRC Press, Boca Raton, FL, pp. 413-434.

Simpson, B.K., Gagne, N., Ashie, I.N., and Noroozi, E. (1997). Utilization of chitozan for preservation of raw shrimp. Food Biotechnol. 11: 25-44.

Simpson, B.K., and Haard, N.F. (1984b). Purification and characterization of trypsin from Greenland cod (Gadus ogac). 1. Kinetic and thermodynamic characteristics. Can. J. Biochem Cel1 Biol. 62: 894.

Simpson, B.K., and Haard, N.F. (1984a). Trypsin from Greenland cod as a food-processing aid. J. Appl. Biochem. 6: 135-143.

Simpson, B.K., and Haard, N.F. (1987). Cold-adapted enzymes from fish. Food Biotechnology. In: Knorr, D. (Ed.). Marcel Dekker, New York, NY, pp. 495-527.

Simpson, B.K., Smith, J.P., and Haard, N.F. (1991). Marine enzymes. Encyclopedia of Food Science and Technology. In: Hui, Y.H. (Ed.). John Wiley and Sons, New York, NY, pp. 1645-1653.

Simpson, K.L. (1982). Carotenoid pigments in seafood. Chemistry and Biochemistry of Marine Food Products. In: Martin, R.E., Flick, G.J., Hebard, C.E., and Ward, D.R. (Ed.). AVI Publishing Co., Inc., Westport, Connecticut, pp. 115-122.

Singh, A., and Benjakul, S. (2018). Proteolysis and its control using protease inhibitors in fish and fish products: A review. Compr. Rev. Food Sci. Food Saf. 17(2): 496-509.

Singh, P., and Nagendran, R. (2016). A comparative study of sorption of chromium (III) onto chitin and chitosan. Appl. Water Sci. 6(2): 199204.

Sinn, N., and Howe, P.R.C. (2008). Mental health benefits of omega-3 fatty acids may be mediated by improvements in cerebral vascular function. Biosci. Hypotheses. 1: 103-108.

Siso, M.I.G., Lang, E., Carreno-Gomez, B., Becerra, M., Espinar, F.O., and Mendez, J.B. (1997). Enzyme Encapsulation on Chitosan Microbeads. Proc. Biochem. 32: 211-216.

Skanderby, M. (1994). Protein hydrolysates: their functionality and applications. Food Technol. Int. Eur. 10: 141-144.

Slizyte, R., Dauksas, E., Falch, E., Storro, I., and Rustad, T. (2005). Characteristics of protein fractions generated from hydrolysed cod (Gadus morhua) by-products. Proc. Biochem. 40: 2021-2033.

Slizyte, R., Mozuraitytè, R., Martínez-Alvarez, O., Fouchereau-Peron, E.F.M., and Rustad, T. (2009). Functional, bioactive and antioxidative properties of hydrolysates obtained from cod (Gadus morhua) backbones. Proc. Biochem. 44: 668-677.

Smine, A., Guérard, F., and Le Gal, Y. (1993). Purification and characterization of pancreatic elastase from tuna (Thunnus albacora). J. Mar. Biotechnol. 1: 41-46.

Smith, T.J., Yank, G.Y., Seril, D.N., Liao, J., and Kim, S. (1998). Inhibition of 4-(methylnitrosamino)-1-(3-pyridyl)-1-butanone-induced lung tumorogenesis by dietary olive oil and squalene. Carcinogenesis. 19: 703-706.

Song, E., Kim, S.Y., Chun, T., Byun, H.J., and Lee, Y.M. (2006). Collagen scaffolds derived from a marine source and their biocompatibility. Biomaterials 27(15): 2951-2961.

Song, W.K., Liu, D., Sun, L.L., Li, B.F., and Hou, H. (2019). Physicochemical and Biocompatibility Properties of Type I Collagen from the Skin of Nile Tilapia (Oreochromis niloticus) for Biomedical Applications. Mar Drugs 17(3): 137.

Soper, J. C., and Thomas, M.T. (2000). U.S. Patent No. 6,039,901. Washington, DC: U.S. Patent and Trademark Office.

Sow, L.C., and Yang, H. (2015). Effects of salt and sugar addition on the physicochemical properties and nanostructure of fish gelatin. Food Hydrocolloids 45: 72-82.

Spinelli, J., and Mahnken, C. (1978). Carotenoid deposition in pen reared salmonids fed diets containing oil extracts of red crab (Pleuronnocodes planipes). Aquacul. 13: 213-216.

Sprague, M., Dick, J.R., and Tocher, D.R. (2016). Impact of sustainable feeds on omega-3 long-chain fatty acid levels in farmed Atlantic salmon, 2006-2015. Sci. Rep. 6: 21892.

Squires, E.J., Haard, N.F., and Feltham, L.A.W. (1986). Pepsin isozymes from Greenland cod, Gadus ogac. 1. Purification and physical properties. Can J Biochem Cell Biol. 65: 205-209.

Sriket, C. (2014). Proteases in fish and shellfish: Role on muscle softening and prevention. Int. Food Res. J. 21(2): 433.

Stepnowski, P., Ólafsson, G., Helgason, H., and Jastorff, B. (2004). Recovery of astaxanthin from seafood wastewater utilizing fish scales waste. Chemosphere. 54: 413-417.

Stoykov, Y.M., Pavlov, A.I., and Krastanov, A.I. (2015). Chitinase biotechnology: production, purification, and application. Eng. Life Sci. 15(1): 30-38.

Suárez-Jiménez, G.M., Robles-Sánches, R.M., Yépiz-Plascencia, G., BurgosHernández, A., and Ezquerra-Brauer, J.M. (2015). In vitro antioxidant, antimutagenic and antiproliferative activities of collagen hydrolysates of jumbo squid (Dosidicus gigas) byproducts. Trends Food Sci. Technol. 35(3): 421-427.

Subhan, F., Ikram, M., Shehzad, A., and Ghafoor, A. (2015). Marine collagen: an emerging player in biomedical applications. J. Food Sci. Technol. 52(8): 4703-4707.

Sudarshan, N.R., Hoover, D.G., and Knorr, D. (1992). Antibacterial action of chitosan. Food Biotechnol. 6: 257-272.

Sugahara, K., and Mikami, T. (2007). Chondroitin/dermatan sulfate in the central nervous system. Curr. Opin. Struct. Biol. 17(5): 536-545.

Sugano, M., Watanabe, S., Kishi, A., Izume, M., and Ohtakara, A. (1998) Hypocholesterolemic action of chitosans with different viscosity in rats. Lipids. 23: 187-191.

Sugano, M., Yoshida, K., Hashimoto, M., Enomoto, K., and Hirano, S. (1992). Hypocholesterolemic activity of partially hydrolyzed chitosans in rats. Advances in chitin and chitosan. In: Brine, C., Zikakis, 
J.P., and Sandford, P. (Ed.). Elsevier, London, UK, pp. 472-478.

Suleria, H., Osborne, S., Masci, P., and Gobe, G. (2015). Marine-based nutraceuticals: An innovative trend in the food and supplement industries. Mar. Drugs 13(10): 6336-6351.

Summers, G. (1987). Squalene, a potential shark by-product. Catch. 14: 29.

Sunil, B.R., and Jagannatham, M. (2016). Producing hydroxyapatite from fish bones by heat treatment. Mater. Lett. 185: 411-414.

Suntornsaratoon, P., Charoenphandhu, N., and Krishnamra, N. (2018). Fortified tuna bone powder supplementation increases bone mineral density of lactating rats and their offspring. J. Sci. Food Agric. 98(5): 2027-2034.

Supavititpatana, P., Wirjantoro, T.I., Apichartsrangkoon, A., and Raviyan, P. (2008). Addition of gelatin enhanced gelation of corn and milk yogurt. Food Chem. 106: 211-216.

Surai, P.F., MacPherson, A., Speake, B.K., and Sparks, N.H. (2001). Designer egg evaluation in a controlled trial. Eur. J. Clin. Nutr. 68: 538-544.

Suresh, G., Das, R.K., Kaur Brar, S., Rouissi, T., Avalos Ramirez, A., Chorfi, Y., and Godbout, S. (2018). Alternatives to antibiotics in poultry feed: molecular perspectives. Crit. Rev. Microbiol. 44(3): 318-335.

Suresh, P.V., Kudre, T.G., and Johny, L.C. (2018). Sustainable Valorization of Seafood Processing By-Product/Discard. Waste to Wealth. Springer, Singapore, pp. 111-139.

Surh, J., Decker, E.A., and McClements, D.J. (2006). Properties and stability of oil-in-water emulsions stabilized by fish gelatine. Food Hydrocolloid. 20: 596-606.

Suryawanshi, N., Jujjavarapu, S.E., and Ayothiraman, S. (2019). Marine shell industrial wastes-an abundant source of chitin and its derivatives: constituents, pretreatment, fermentation, and pleiotropic applications-a revisit. Int. J. Environ. Sci. Technol. 16(7): 3877-3898.

Suzuki, K., Mikami, T., Okawa, Y., Tokoro, A., Suzuki, S., and Suzuki, M. (1986). Antitumor effect of hexa- $N$-acetylchitohexaose and chitohexaose. Carbohydr Res. 151: 403-408.

Swanson, D., Block, R., and Mousa, S.A. (2012). Omega-3 fatty acids EPA and DHA: health benefits throughout life. Adv. Nutr. 3(1): 1-7.

Swern, D. (1964). Technique of Separation: Urea Complexation. Fatty Acids. In: Markley, K.S. (Ed.). Interscience Publishers, New York, NY, USA, pp. 2309-2358.

Synowiecki, J., and Al-Khateeb, N.A. (2003). Production, properties, and some new applications of chitin and its derivatives. Crit. Rev. Food Sci. Nutr. 43(2): 145-171.

Synowiecki, J., Sikorski, Z.E., Naczk, M., and Piotrzkowska, H. (1982). Immobilization of Enzymes on Krill Chitin Activated by Formaldehyde. Biotechnol. Bioeng. 24: 1871-1876.

Tümerkan, E.T.A., Cansu, Ü., Boran, G., Mac Regenstein, J., and Özoğul, F. (2019). Physiochemical and functional properties of gelatin obtained from tuna, frog and chicken skins. Food Chem. 287: 273-279.

Tahergorabi, R., and Ibrahim, S. (2016). Functional Food Product Development from Fish Processing By-products Using Isoelectric Solubilization/Precipitation. In Proceedings of the 2013 National Conference on Advances in Environmental Science and Technology. Springer, Cham, pp. 179-183.

Tai, E.K., Wang, X.B., and Chen, Z.Y. (2013). An update on adding docosahexaenoic acid (DHA) and arachidonic acid (AA) to baby formula. Food Funct. 4(12): 1767-1775.

Takahashi, T., Imai, M., Suzuki, I., and Sawai, J. (2008). Growth inhibitory effect on bacteria of chitosan membranes regulated with deacetylation degree. Biochem. Eng. J. 40(3): 485-491.

Takahashi, Y., Miki, F., and Nagase, K. (1995). Effect of Sonolysis on Acid Degradation of Chitin to Form Oligosaccharides. Bull. Chem. Soc. Jpn. 68: 1851-1857.

Takamatsu, T. (1994). Hydrocarbons. Cosmetic Raw Material Analysis and Quality. In: Butler, H. (Ed.). Micelle Press, pp. 24-31.

Takeungwongtrakul, S., Benjakul, S., Santoso, J., Trilaksani, W., and NuriImala, M. (2015). Extraction and Stability of Carotenoid-Containing Lipids from Hepatopancreas of P acific White Shrimp (Litopenaeus vannamei). J. Food Process. Preserv. 39(1): 10-18.

Tanaka, Y., Katoyose, T., and Katayama, T. (1981). Changes of carotenoids in Antarctic krill meal during storage. Memoirs Fac Fish Kagoshima Univ. 30: 295-299.

Tanaka, Y., Tanioka, S., Tanaka, M., Tanigawa, T., Kitamura, Y., Minami, S., Okamoto, Y., Miyashita, M., and Nanno, M. Effects of chitin and chi- tosan particles on BALB/c mice by oral and parenteral administration. Biomater. 18: 591-595.

Tang, S., Dong, S., Chen, M., Gao, R., Chen, S., Zhao, Y., and and Sun, B. (2018). Preparation of a fermentation solution of grass fish bones and its calcium bioavailability in rats. Food Funct. 9(8): 4135-4142.

Taniguchi, A., Takano, K., and Kamoi, I. (2001). Purification and properties of lipase from Tilapia intestine-digestive enzyme of Tilapia-VI. Nippon Suisan Gakk. 67: 78-84.

Tanii, T., Katoh, J., Yashiro, N., Shindo, K., Kono, T., Hamada, T., and Yamaguchi, M. (1991). Clinical evaluations of squalane in patients with xerotic dermatoses and the results of patch test of squalane and its moisturizing effects. Skin Res. 33(2): 155-163.

Taskaya, L., and Jaczynski, J. (2009). Flocculation-enhanced protein recovery from fish processing by-products by isoelectric solubilization/ precipitation. Food Sci. Technol. 42: 570-575.

Taskayaa, L., and Jaczynski, J. (2009). Flocculation-enhanced protein recovery from fish processing by-products by isoelectric solubilization/ precipitation. Food Sci. Technol. 42: 570-575.

Tatterson, I.N. (1982). Fish silage-preparation, properties and uses. Anim. Feed Sci. Technol. 7: 153-159.

Taylor, S.L. (2009). Advances in food and nutrition research. Advances in food and nutrition research. 58:

Taylor, T., and Alasalvar, C. (2002). Improved utilisation of fish and shellfish waste. Seafoods-Quality, Technology and Nutraceutical Applications. In: Cesarettin, A., and Tony, T. (Ed.). Springer, Berlin, Heidelberg, Germen, pp. 123-136.

Te Nijenhuis, K. (1997). Thermoreversible networks: viscoelastic properties and structure of gels. Adv. Polym. Sci. 130: 160-193.

Teo, L.H., and Sabapathy, U. (1990). Preliminary report on the digestive enzymes present in the digestive gland of Perna viridis. Mar. Biol. 106: 403-407.

Tharanathan, R.N. (2002). Food-derived carbohydrates-structural complexity and functional diversity. Critical reviews in biotechnology 22(1): 65-84

Thatcher, W.W., and Staples, C.R. (2000). Effects of dietary fat supplementation on reproduction in lactating dairy cows. Adv. Dairy Technol. 12: $213-232$

Tischer, W., and Wedekind, F. (1999). Immobilized enzymes: methods and applications. Top. Curr. Chem. 200: 95-126.

Tocher, D.R., and Sargent, J.R. (1984). Studies on triacylglycerol, wax ester, and sterol ester hydrolases in intestinal caeca of rainbow trout (Salmo gairdneri) fed diets rich in triacylglycerols and wax esters. Comp. Biochem. Physiol., Part B: Biochem. Mol. Biol. 77: 561-571.

Tolaimate, A., Desbrieres, J., Rhazi, M., and Alagui, A. (2003). Contribution to the preparation of chitins and chitosans with controlled physicochemical properties. Polymer 44(26): 7939-7952.

Tomihata, K., and Ikada, Y. (1997). Degradation of chitin and its deacetylated derivatives. Biomater. 18: 567-575.

Torres, J.A., Chen, Y.C., Rodrigo-Garcia, J., and Jaczynski, J. (2007). Recovery of by-products from seafood processing streams. Maximising the value of marine by-products. In: Shahidi, F. (Ed.). CRC Press, Boca Raton, USA, pp. 65-90.

Torres, J.A., Chen, Y.C., Rodrigo-Garcia, J., and Jaczynski, J. (2007). Recovery of byproducts from seafood processing streams. Maximising the value of marine by-products. In: Shahidi, F. (Ed.). CRC Press, Boca Raton, FL, pp. 65-90.

Torrissen, O., Tidemann, E., Hansen, F., and Raa, J. (1982). Ensiling in acid - a method to stabilize astaxanthin in shrimp processing byprod ucts and improve uptake of this pigment by rainbow trout (Salmo gairdneri). Aquacul. 26: 77-83.

Torrissen, O.J. (1986). Pigmentation of salmonids-a comparison of astaxanthin and canthaxanthin as pigment sources for rainbow trout. Aquacul. 53: 271-278.

Tsai, G.J., and Su, W.H. (1999). Antibacterial activity of shrimp chitosan against Escherichia coli. J. Food. Prot. 62: 239-243.

Tsai, G.J., Su, W.H., Chen, H.C., and Pan, C.L. (2002). Antimicrobial activity of shrimp chitin and chitosan from different treatments and applications of fish preservation. Fisheries Sci. 68(1): 170-177.

Tsujimoto, M. (1932). The Liver Oils of Elasmobranch Fish. J. Sci. Chem. Ind. (Trans) 51: 317-323.

Tsukada, K., Matsumoto, T., Aizawa, K., Tokoro, A., Naruse, R., Suzuki, S., 
and Suzuki, M. (1990). Antimetastatic and growth-inhibitory effects of $\mathrm{N}$-acetylchitohexaose in mice bearing Lewis lung carcinoma. Jap. J. Cancer Res. 81: 259-265.

Turan, H., and Sonmez, G. (2007). Changes in the quality of surimi made from thornback ray (Raja clavata, L. 1758) during frozen storage. Int. J. Food Sci. Nutr. 58: 557-66.

Tweedie, E.P., Coblentz, F.E., and Shafer, T.H. (2004). Purification of a soluble glycoprotein from the uncalcified ecdysial cuticle of the blue crab Callinectes sapidus and its possible role in initial mineralization. J. Exp. Biol. 207(15): 2589-2598.

Tziveleka, L.A., loannou, E., Tsiourvas, D., Berillis, P., Foufa, E., and Roussis, V. (2017). Collagen from the marine sponges Axinella cannabina and Suberites carnosus: Isolation and morphological, biochemical, and biophysical characterization. Mar. Drugs 15(6): 152.

Uauy, R.D. (1990). Are omega 3 fatty acids required for normal; eye and brain development in the human? J. Pediar. Gastroenterol. Nutr. 11: 296-302.

Uauy, R.D., Birch, D.G., Birch, E.E., Tyson, J.E., and Hoffman, D.R. (1990). Effect of dietary omega 3 fatty acids on retinal function of very-lowbirth weight neonates. Pediat. Res. 28: 485-492.

Uchida, Y., Izume, M., and Ohtakara, A. (1989). Preparation of oligomers with purified chitosanase and its application. Chitin and Chitosan. In: Skjak-Braek, G., Anthonsen, T., and Sandford, P. (Ed.). Elsevier, London, pp. 373-382.

Umayaparvathi, S., Meenakshi, S., Vimalraj, V., Arumugam, M., Sivagami, G., and Balasubramanian, T. (2014). Antioxidant activity and anticancer effect of bioactive peptide from enzymatic hydrolysate of oyster (Saccostrea cucullata). Biomed. Prev. Nutr. 4(3): 343-353.

UNEP. www.grid.unep.ch/ew.

Usui, T., Hayashi, Y., Nanjo, F., Sakai, K., and Ishido, Y. (1987). Transglycosylation reaction of a chitinase purified from Nocardia orientalis. Biochim. Biophys. Acta. 923: 302-309.

Usui, T., Matsui, H., and Isobe, K. (1990). Enzymic synthesis of useful chitooligosaccharides utilizing transglycosylation by chitinolytic enzymes in a buffer containing ammonium sulphate. Carbohydr. Res. 203: $65-77$.

Uzel, S.G., and Buehler, M.J. (2009). Nanomechanical sequencing of collagen: tropocollagen features heterogeneous elastic properties at the nanoscale. Integr. Biol. 1(7): 452-459.

Vázquez, J.A., Blanco, M., Fraguas, J., Pastrana, L., and Pérez-Martín, R. (2016). Optimisation of the extraction and purification of chondroitin sulphate from head by-products of Prionace glauca by environmental friendly processes. Food Chem. 198: 28-35.

Vázquez, J.A., Meduíña, A., Durán, A.I., Nogueira, M., Fernández-Compás, A., Pérez-Martín, R.I., and Rodríguez-Amado, I. (2019). Production of Valuable Compounds and Bioactive Metabolites from By-Products of Fish Discards Using Chemical Processing, Enzymatic Hydrolysis, and Bacterial Fermentation. Mar. Drugs 17(3): 139.

Vázquez, J.A., Nogueira, M., Durán, A., Prieto, M.A., Rodríguez-Amado, I., Rial, D., González, M.P., and Murado, M.A. (2011). Preparation of marine silage of swordfish, ray and shark visceral waste by lactic acid bacteria. J. Food Eng. 103(4): 442-448.

Valcarcel, J., Novoa-Carballal, R., Pérez-Martín, R.I., Reis, R.L., and Vázquez, J.A. (2017). Glycosaminoglycans from marine sources as therapeutic agents. Biotechnol. Adv. 35(6): 711-725.

van de Velde, F., Lourenço, N.D., Pinheiro, H.M., and Bakker, M. (2002). Carrageenan: a food-grade and biocompatible support for immobilisation techniques. Adv. Synth. Catal. 344: 815-835.

van Haaren, E.H., Smit, T.H., Phipps, K., Wuisman, P.I.J.M., Blunn, G., and Heyligers, I.C. (2005). Tricalcium-phosphate and hydroxyapatite bone-graft extender for use in impaction grafting revision surgery. Brit. J. Bone Joint Surg. 87: 267-271.

Vani, R., and Stanley, S.A. (2013). Studies on the extraction of chitin and chitosan from different aquatic organisms. Advanced BioTech 12(12): 12-15.

Velazquez, G., Miranda-Luna, P., Lopez-Echevarria, G., Vazquez, M., Torres, J.A., and Ramırez, J.A. (2008). Effect of Pacific whiting wash water proteins on Alaska pollock surimi gels. J. Texture Stud. 39: 296-308.

Venkatesan, J., Lowe, B., Manivasagan, P., Kang, K.H., Chalisserry, E., Anil, S., Kim, D., and Kim, S.K. (2015). Isolation and characterization of nanohydroxyapatite from salmon fish bone. Materials 8(8): 5426-5439.
Venugopal, V. (1995). By-products from industrial fishery processing. Ind. Food Indus. 14: 22-24.

Venugopal, V. (2016). Enzymes from seafood processing waste and their applications in seafood processing. Adv. Food Nutr. Res. 78: 47-69.

Venugopal, V. (1997). Functionality and potential applications of thermostable water dispersions of fish meat. Trends Food Sci Technol. 8: 271-276.

Venugopal, V., and Shahidi, F. (1998). Traditional methods to process underutilized fish species for human consumption. Food Rev. Int. 14: 35-97.

Venugopal, V., and Shahidi, F. (1995). Value-added products from underutilized fish species. Crit. Rev. Food Sci. Nutr. 35: 431-453.

Wanasundara, U.N., and Shahidi, F. (1999). Concentration of omega 3-polyunsaturated fatty acids of seal blubber oil by urea complexation: optimization of reaction conditions. Food Chem. 65: 41-49.

Wanasundara, U.N., and Shahidi, F. (1997). Lipase Assisted Concentration of $\omega 3$-Polyunsaturated Fatty Acids in Acylglycerols Form from Marine Oils. J. Am. Oil Chem. Soc. 75: 945-951.

Wanasundara, U.N., Shahidi, F., and Amarowicz, R. (1998). Effect of processing on constituents and oxidative stability of marine oils. J. Food Lipids 5: 29-41.

Wang, A.C.A., Khor, E., and Hastings, G.W. (1997). Hydroxyapatite modified chitin as potential hard tissue substitute material. J. Biomed. Mater. Res. 38: 235-241.

Wang, C., Chang, T., Shi, L., Yang, H., Cui, M., and Tambalu, L. (2014). Seafood processing by-products: collagen and gelatin. Seafood Processing By-Products. Springer, New York, NY, pp. 207-242.

Wang, G.H. (1992). Inhibition and inactivation of five species of food borne pathogens by chitosan. J. Food Prot. 55: 916-919.

Wang, Z., Cai, C.F., Cao, X.M., Zhu, J.M., He, J., Wu, P., and Ye, Y.T. (2018). Supplementation of dietary astaxanthin alleviated oxidative damage induced by chronic high pH stress, and enhanced carapace astaxanthin concentration of Chinese mitten crab Eriocheir sinensis. Aquaculture 483: 230-237.

Wang, Z., Qiao, Y., Huang, G.S., Wang, A.Q., Zhang, Y.Q., Feng, J.L., Yang, G.R., Guo, Y., and Liang, R. (2003a). Glucosamine and glucosamine hydrochloride induced leukemia cell line K562 differentiation into macrophage. Chinese Pharmacol. Bull. 19: 290-293(in Chinese).

Wang, Z., Qiao, Y., Huang, G.S., Wang, A.Q., Zhang, Y.Q., Feng, J.L., Yang, G.R., Guo, Y., and Liang, R. (2003b). Induction of macrophagic differentiation of leukemia cell line K562 by N-acetyl-D-glucosamine. J. Fourth Milit. Med. Univ. 24: 46-48(in Chinese).

Wang, Z., Taylor, K.D., and Yan, X. (1992). Studies on the protease activities in Norway lobster (Nephrops norvegicus) and their role in the phenolamin activation process. Food Chem. 42: 111-116.

Wasswa, J., Tang, J., and Gu, X. (2007). Utilization of fish processing byproducts in the gelatine industry. Food Rev. Int. 23: 159-174.

Watanabe, Y. (2001). Bycatch abd discard fish in the Japanese fisheries. More Efficient Utilization of Fish and Fisheries Products. In: Sakaguchi, M. (Ed.). Elsevier Ltd., pp. 25-34.

Watson, R.R. (Ed.). (2002). Eggs and health promotion. lowa State Press, a Blackwell Publishing Company, Ames USA, pp. 19-36.

White, A., Handler, P., and Smith, E.L. (1968). Principles of biochemistry. McGraw-Hill, New York, 4th Ed.

WHO. http://www.who.int/vaccine_safety/topics/adjuvants/squalene.

Wong, D.W.S. (1995). Proteolytic enzymes. Food enzymes; structure and mechanism. Champman and Hall, New York, p. 147.

Wong, H., and Schotz, M.C. (2002). The lipase gene family. J. Lipid Res. 43: 993-999.

Wu, G.P., Chen, S.H., Liu, G.M., Yoshida, A., Zhang, L.J., Su, W.J., and Cao, M.J. (2010). Purification and characterization of a collagenolytic serine proteinase from the skeletal muscle of red sea bream (Pagrus major). Comp. Biochem. Physiol. B: Biochem. Mol. Biol. 155(3): 281-287.

Wu, H.C., Chen, H.M., and Shiau, C.Y. (2003). Free amino acids and peptides as related to antioxidant properties in protein hydrolysates of mackerel (Scomber austriasicus). Food Res. Int. 36: 949-957.

Wu, J., Guo, X., Liu, H., and Chen, L. (2019). Isolation and Comparative Study on the Characterization of Guanidine Hydrochloride Soluble Collagen and Pepsin Soluble Collagen from the Body of Surf Clam Shell (Coelomactra antiquata). Foods 8(1): 11 . 
Wu, J.L., Lu, B.J., Du, M.H., Liu, G.M., Hara, K.J., Su, W.J., and Cao, M.J. (2008). Purification and characterization of gelatinase-like proteinases from the dark muscle of common carp (Cyprinus carpio). J. Agric. Food. Chem. 56(6): 2216-2222.

Xu, X., Dong, J., Mu, X., and Sun, L. (2011). Supercritical CO2 extraction of oil, carotenoids, squalene and sterols from lotus (Nelumbo nucifera Gaertn) bee pollen. Food Bioprod. Process. 89(1): 47-52.

Yalpani, M., Johnson, F., and Robinson, L.E. (1992). Antimicrobial activity of some chitosan derivatives. Advances in Chitin and Chitosan. In: Brine, C.J., Sandford, P.A., and Zikakis, J.P. (Ed.). Elsevier, London, UK, pp. 543-548.

Yan, M., Li, B., and Zhao, X. (2009). Isolation and characterization of collagen from squid (Ommastrephes bartrami) skin. J. Ocean U. China 8(2): 191-196

Yan, M.Y., Li, B.F., Zhao, X., Ren, G.Y., Zhuang, Y.L., Hou, H., Zhang, X.K., Chen, L., and Fan, Y. (2008). Characterization of acid-soluble collagen from the skin of walleye pollock (Theragra chalcogramma). Food Chem. 107: 1581-1586.

Yang, H., Wang, Y., Jiang, M., Oh, J.H., Herring, J., and Zhou, P. (2007). 2 step optimization of the extraction and subsequent physical properties of Channel Catfish (Ictalurus punctatus) skin gelatine. J. Food Sci. 72: C188-C195.

Yang, L.Y., Kuksis, A., and Myher, J.J. (1990). Lipolysis of menhaden oil triacylglycerols and the corresponding fatty acid alkyl esters by pancreatic lipase in vitro: a reexamination. J. Lipid Res. 31(1): 137-147.

Ylitalo, R., Lehtinen, S., Wuolijoki, E., Ylitalo, P., and Lehtimaki, T. (2002). Cholesterol lowering properties and safety of chitosan. Drug res. 1: $1-7$.

Yongmanitchai, W., and Ward, O.P. (1989). Omega-3 fatty acids: Alternative sources of production. Prog. Biochem. 24: 117-125.

Yoshimura, K., Terashima, M., Hozan, D., Ebato, T., Nomura, Y., and Ishii, Y. (2000). Physical properties of shark gelatine compared with pig gelatine. J. Agri. Food Chem. 48: 2023-2027.

Younes, I., Sellimi, S., Rinaudo, M., Jellouli, K., and Nasri, M. (2014). Influence of acetylation degree and molecular weight of homogeneous chitosans on antibacterial and antifungal activities. Int. J. Food Microbiol. 185: 57-63.

Young, A.J., and Lowe, G.M. (2001). Antioxidant and prooxidant properties of carotenoids. Arch. Biochem. Biophys. 385(1): 20-27.

Young, D.H., and Kauss, H. (1983). Release of calcium from sus- pensioncuitured glycine max cells by chitosan, other polycations, and polyamines in relation to effects on membrane permeability. Plant Physiol. 73: 698-702.

Yu, L.J., and Brooks, M.L. (2016). Food industry protein by-products and their applications. Protein byproducts. Academic Press, pp. 120-132.

Yuan, Y.V., and Kitts, D.D. (1994). Calcium absorption and bone utilization in spontaneously hypertensive rats fed on native and heat-damaged casein and soya-bean protein. Br. J. Nutr. 71(4): 583-603.

Yuan, Y.V., and Kitts, D.D. (1991). Confirmation of calcium absorption and femoral utilization in spontaneously hypertensive rats fed casein phosphopeptide supplemented diets. Nutr. Res. 11(11): 1257-1272.

Yuji, M., Keisuke, T., Yasue, N., Yoshiyuki, K., Makoto, A., Takashi, T., Wataru, T., Atsushi, T., Hiroyoshi, H., and Tomotari, M. (1993). Hypocholes- terolemic effect of chitosan in adult males. Biosci. Biotech. Biochem 57: 1439-1444.

Zaheer, K. (2015). An updated review on chicken eggs: production, consumption, management aspects and nutritional benefits to human health. Food Nutr. Sci. 6(13):

Zamani, A., Jeihanipour, A., Edebo, L., Niklasson, C., and Taherzadeh, M.J. (2008). Determination of Glucosamine and N-Acetyl Glucosamine in Fungal Cell Walls. J. Agric. Food Chem. 56: 8314-8318.

Zambonin, G., and Grano, M. (1995). Biomaterials in orthopaedic surgery: effects of different hydroxyapatites and demineralized bone matrix on proliferation rate and bone matrix synthesis by human osteoblasts. Biomater. 16: 397-402.

Zamora-Sillero, J., Gharsallaoui, A., and Prentice, C. (2018). Peptides from fish by-product protein hydrolysates and its functional properties: an overview. Mar. Biotechnol. 20(2): 118-130.

Zefirova, O.N., Mamaeva, A.V., Chupov, V.V., Valuev, L.I., and Plate, N.A (1996). Synthesis and properties of immobilized collagenolytic protease from hepatopancreas of the king crab Paralithoides camtschatica. Appl. Biochem. Microbiol. 32: 461-464.

Zeller, D., Cashion, T., Palomares, M., and Pauly, D. (2018). Global marine fisheries discards: A synthesis of reconstructed data. Fish and Fisheries, FAO 19(1): 30-39.

Zhang, F., Wang, A., Li, Z., He, S., and Shao, L. (2011). Preparation and characterisation of collagen from freshwater fish scales. Food Sci. Nutr. 2(08): 818.

Zhang, H., Xiong, Y., Bakry, A.M., Xiong, S., Yin, T., Zhang, B., Huang, J., Liu, Z., and Huang, Q. (2019). Effect of yeast $\beta$-glucan on gel properties, spatial structure and sensory characteristics of silver carp surimi. Food Hydrocolloids 88: 256-264.

Zhang, H.J., Luo, C.X., Zhang, X.S., Song, M.Z., and Jiang, X.P. (2003). Application of collagen protein. Leather Sci. Eng. 13: 37-46(in Chinese with English abstract).

Zhang, L., Liu, W.S., Han, B.Q., Peng, Y.F., and Wang, D.F. (2006). Antitumor activities of D-glucosamine and its derivatives. J. Zhejiang Univ. Sci. B. 7: 608-614.

Zhao, H., Cui, Q., Shah, V., Xu, J., and Wang, T. (2016). Enhancement of glucose isomerase activity by immobilizing on silica/chitosan hybrid microspheres. J. Mol. Catal. B: Enzym. 126: 18-23.

Zhou, P., Mulvaney, S.J., and Regenstein, J.M. (2006). Properties of Alaska pollock skin gelatin: a comparison with tilapia and pork skin gelatins. J. Food Sci. 71(6): C313-C321.

Zhou, P., and Regenstein, J.M. (2005). Effects of alkaline and acid pretreatments on Alaska pollock skin gelatine extraction. J. Food Sci. 70: C392-C396.

Zhou, P., and Regenstein, J.M. (2004). Optimization of extraction conditions for pollock skin gelatine. J. Food Sci. 70: C393-C398.

Zhuang, Y., Li, B., and Zhao, X. (2009). The scavenging of free radical and oxygen species activities and hydration capacity of collagen hydrolysates from walleye pollock (Theragra chalcogramma) skin. J. Ocean Univ. China (English Edition). 8: 171-176

Ziegler, F., Winther, U., Hognes, E.S., Emanuelsson, A., Sund, V., and Ellingsen, $H$. (2013). The carbon footprint of Norwegian seafood products on the global seafood market. J. Ind. Ecol. 17(1): 103-116. 